Accepted 2020 December 15, Received 2020 October 13 and scheduled for publication in the Monthly Notices

\title{
Detection of a High-Temperature Blackbody Hump in Black Hole Spectra. The strongly redshifted annihilation line
}

\author{
Lev Titarchuk ${ }^{1,2}$ and Elena Seifina ${ }^{2,3}$
}

\begin{abstract}
We discovered a so called high-temperature blackbody (HBB) component, found in the 15 - $40 \mathrm{keV}$ range, in the broad-band X-ray energy spectra of black hole $(\mathrm{BH})$ candidate sources. A detailed study of this spectral feature is presented using data from five of the Galactic BH binaries, Cyg X-1, GX 339-4, GRS 1915+105, SS 433 and V4641 Sgr in the low/hard, intermediate, high/soft and very soft spectral states (LHS, IS, HSS and VSS, respectively) and spectral transitions between them using RXTE, INTEGRAL and BeppoSAX data. In order to fit the broad-band energy spectra of these sources we used an additive XSPEC model, composed of the Comptonization component and the Gaussian line component. In particular, we reveal that the IS spectra have the HBB component which color temperature, $k T_{\mathrm{HBB}}$ is in the range of $4.5-5.9 \mathrm{keV}$. This HBB feature has been detected in some spectra of these five sources only in the IS (for the photon index $\Gamma>1.9$ ) using different X-ray telescopes. We also demonstrate that a timescale of the HBB-feature is of orders of magnitude shorter than the timescale of the iron line and its edge. That leads us to conclude that these spectral features are formed in geometrically different parts of the source and which are not connected to each other. Laurent \& Titarchuk (2018) demonstrated a presence of a gravitational redshifted annihilation line emission in a $\mathrm{BH}$ using the Monte-Carlo simulations and therefore the observed HBB hump leads us to suggest this feature is a gravitational redshifted annihilation line observed in these black holes.
\end{abstract}

\footnotetext{
${ }^{1}$ Astro Space Center, Lebedev Physical Institute, Russian Academy of Sciences, Profsouznay ul. 84/32, Moscow 117997, Russia; ltitarchuk@asc.rssi.ru; titarchuk@fe.infn.it

${ }^{2}$ Lomonosov Moscow State University/Sternberg Astronomical Institute, Universitetsky Prospect 13, Moscow, 119992, Russia; seif@sai.msu.ru

${ }^{3}$ LAPTh, Universite Savoie Mont Blanc, CNRS, B.P. 110, Annecy-le-Vieux F-74941, France; All-Russian Institute of Scientific and Technical Information, RAS, Usievich st. 20, Moscow, 125190, Russia
} 
Subject headings: accretion, accretion disks - black hole physics — stars:individual (Cyg X-1, GX 339-4, GRS 1915+105, SS 433, V4641 Sgr):radiation mechanisms: non-thermal - physical data and processes

\section{Introduction}

High-energy astrophysics of compact objects offers a unique opportunity to take a peek at the properties of matter under the most extreme conditions because they produce a variety of observational manifestations which can be used to verify a theoretical model. The progress of the theoretical physics studying matter under extreme conditions is essential for creating correct models of compact objects and for adequate interpretation of available observations.

A study of the spectral variability properties of X-ray binaries is a valuable source of information on the physics of accretion processes and the fundamental parameters of a black hole $(\mathrm{BH})$. Among the observable signatures of a $\mathrm{BH}$ was a discovery of the photon index versus quasi-periodic oscilation (QPO) frequency (and mass accretion rate) correlations followed by the index saturation [see Shaposhnikov \& Titarchuk, 2006; 2009, hereafter ST09]. These correlations were revealed during the spectral transitions from the low/hard state (LHS) through the intermediate state (IS) to the high soft state (HSS) in X-ray binaries (see, for example a review by McClintock et al. 2009).

In Galactic and extragalactic BHs, the X-ray spectra in the HSS are dominated by a thermal blackbody (BB) component that peaks in the $0.1-2 \mathrm{keV}$ range and in addition, these BHs show a strong power-law component in the LHS. Thus the energy spectrum is dominated by a hard Comptonization component convolved with a weak thermal component in the LHS. The spectra of BHs in the LHS are presumably a result of the thermal Comptonization (upscattering) of soft photons, originated in a relatively weak accretion disk, off electrons of the hot ambient plasma (see, e.g., Sunyaev \& Titarchuk 1980 and Titarchuk 1994, hereafter ST80 and T94, respectively). The IS is a transitional state of the source when it undergoes a spectral transition between the LHS and HSS. The IS spectra are usually characterized by the photon indices about 2 or higher than that. The soft BB component of these spectra is interpreted in terms of a geometrically thin, optically thick accretion disk around the compact object (see Shakura \& Sunyaev 1973, hereafter SS73). Energy dissipation within the disk should be responsible for the observed soft BB emission. The Comptonization continuum is believed to originate in the Compton cloud (CC) due to upscattering of the thermal disk photons by the electrons of a hot corona or a hot ambient plasma (ST80; Haardt 1993; Dove et al. 1997; Dauser et al. 2013). In addition to the LHS, IS and HSS sometimes very soft 
state (VSS) is observed in which the BB component is dominated in the source spectrum and the power-law (PL) component is either very weak or absent at all. Possibly, in this state, the PL component is screened from the observer by the disk body, which is why the spectrum becomes so soft. The bolometric luminosity in the VSS is a factor of 2 lower than that in HSS.

There is also an interpretation that some of these spectra consist of a so called reflection component which is formed due to downscattereding of the Comptonization radiation in the relatively cold accretion disk. The X-ray reflection effect from accretion disk or relatively cold atmosphere of a companion in the close binary has been studied for more than four decades (see, e.g., Basko et al. 1974; McClintock et al. 2014; Ludlam et al. 2017a,b).

The current paradigm is that the original power-law radiation illuminates the surface of the accretion disk. Then the X-ray photons interact with the material providing diverse atomic features. These can be caused by both via absorption (in most cases as edges) and emission (in a form of fluorescence lines and radiative recombination continuum). For example, the reflection (downscattering) component can provide a direct information about structure, temperature, ionization stage, and composition of the gas in the accretion disk [see also the recent papers on this subject by Garcia et al. (2013); Tomsick et al. (2014) and Walton et al. (2016)]. Sometimes, the presence of the Fe K-shell fluorescence emission and the absorption K-edge observed in the $6-8 \mathrm{keV}$ energy range can be indicative to the reflection effect.

Furthermore, it was suggested that this reflection effect occurs near a $\mathrm{BH}$ and the line photons suffer Doppler effects, light bending, and gravitational redshift, which produce a skewed line profile with a red wing that can be extended to lower energies (e.g., Fabian et al. 2000; Fabian \& Vaughan 2003; Reynolds \& Nowak 2003; Dovčiak et al. 2004; Miller et al. 2008; Steiner et al. 2011; Reynolds et al. 2012; Dauser et al. 2012). However, Laurent \& Titarchuk (2007), hereafter LT07, (§3.2 there), study in detail an effect of the downscattering and analytically prove that the characteristic downscattering hump is only formed when the spectral index, $\alpha$ of the incident spectrum is essentially less than one (or the photon index $\Gamma<2$ ), see also an illustration of this effect in ST80, Fig. 10 there. Thus all possible detections of so called the reflection (or downscatered) hump in the case of $\alpha>1$ (or $\Gamma>2$ ) require a different interpretation.

Laurent \& Titarchuk (2018), hereafter LT18 argued that the photon-photon interactions occurred in the vicinity of a $\mathrm{BH}$ should lead to the pair production there because photon energies reach a limit of $511 \mathrm{keV}$. The created positrons interact with accreting electrons and as a result the annihilation line photons are generated over a relatively narrow shell which is a few hundred meters near a BH horizon for a 10 solar mass BH. An Earth observer can 
see this annihilation line only as a broad spectral feature at the gravitationally redshifted energies with the red shift, $\mathrm{z}$ about 20 since the line is formed over the hundred meter shell near a BH horizon. As a result the observed line energies $E_{\text {obs }} \sim 511 \mathrm{keV} / 20 \sim 20-40 \mathrm{keV}$. The shape of this annihilation line feature can be approximated by a hot blackbody (HBB) of a color temperature, $k T_{H B B} \sim 4-6 \mathrm{keV}$. However, the line can be smeared out due to multiple scattering if the Klein-Nishina optical depth of the converging flow at $511 \mathrm{keV}$ is greater than 2 which unavoidably complicates its detection.

This HBB, additional to the Comptonized emission and iron line components were reported in a number of Galactic microquasars. Specifically, Titarchuk \& Seifina (2009; hereafter TS09), found that eight intermediate state (IS) spectra of GRS 1915+105 (during the 2005-2006 flaring events) required this HBB component. Furthermore, Seifina \& Titarchuk (2010; hereafter ST10) found 24 spectra of SS 433 in the IS during radio outburst decay which had a strong HBB of color temperatures of $4-6 \mathrm{keV}$.

A similar HBB components have been detected from other BHs. In particular, Koljonen et al. (2013) analyzed X-ray data of Cyg X-3 obtained by Swift, RXTE and INTEGRAL during the 2006 flaring episodes (see also Shrader et al. 2010). They found that X-ray variability during major flaring events can be related to the Comptonization and HBB components. It is interesting that this variability of the HBB component correlates with a change of X-ray and radio fluxes.

Tomsick et al. (2005) reported a strong hump at $\sim 20 \mathrm{keV}$ in some RXTE spectra of $4 \mathrm{U}$ 1630-47, which could not be satisfactorily fitted using their spectral model which was consist of the multicolor disk, cutoff power-law and Gaussian component. Later, Seifina et al. (2014) also found a small positive excess at $20 \mathrm{keV}$ but for only one particular RXTE observation (ID = 50135-02-03-00) previously studied by Tomsick et al..

Mineo et al. (2012) revealed that X-ray spectra in the so called heartbeat state of GRS $1915+105$ can be fitted by a model which includes the HBB of a color temperature of $3-6 \mathrm{keV}$. The spectra from BH soft X-ray transients, GS 2000+25, GS 1124-68 and XTE J1550-564 were successfully approximated by an additional thermal Comptonization component which color temperatures are in the $2-4 \mathrm{keV}$ range and high optical depth $\tau \sim 5$ (Zycki, Done \& Smith 2001). These findings raise a fair question on the nature of the HBB component as an intrinsic property of BHs.

We present a further study of this spectral feature using the data of RXTE, INTEGRAL and BeppoSAX for the Galactic BHs: Cyg X-1, GX 339-4, GRS 1915+105, SS 433 and V4641 Sgr during their state transition from the LHS to the HSS through the IS. We investigate variability of spectral properties in short time scales, particularly for GRS 1915+105. 
We focus on the LHS-IS transitions. As we have already aforementioned, during such a transition phase, TS09 registered a broad excess hump centered at $20 \mathrm{keV}$ for the RXTE energy spectra of GRS 1915+105 and later, ST10 found this feature in the RXTE and INTEGRAL spectra of SS 433 with time exposures up to $\sim 3 \mathrm{ksec}$. It is interesting how these spectral characteristics change for a shorter exposition.

As we have already pointed out, the HBB humps in X-ray spectra are well known for many BHs [Zycki et al. (2001); Koljonen etal. (2013); Mineo et al. (2012), Tomsick et

al. (2005), TS09, ST10]. However, the nature of these humps can be different depending on physical conditions.

In this paper we concentrate our efforts to demonstrate an observational manifestation of the HBB hump in X-ray spectra of five BH binaries, Cyg X-1, GX 339-4, GRS 1915+105, SS 433 and V4641 Sgr. In $\S 2$ we provide details of the data analysis while in $\S 3$ we present description of the spectral models used for fitting of these data and provide a reader details of timing analysis. In $\S \S 4-5$ we focuse on observational results and their interpretation. In $\S 6$ we discuss the main results of the paper. In $\S 7$ we make our final conclusions.

\section{Observations and Data Reduction}

For a study presented in this paper we have analyzed archival data for five $\mathrm{BH}$ transient sources, Cyg X-1, GX 339-4, GRS 1915+105, SS 433, and V4641 Sgr, observed with RXTE. We used data from the Proportional Counter Array (PCA) and High-Energy X-Ray Timing Experiment (HEXTE) on board of the RXTE (Bradt et al. 1993). In Tables $1-4$ we give a general information on each observation used in our analysis. In the paper we also used the results of our previous investigations (ST10 and TS09) based on the archival RXTE data for SS 433, V4641 Sgr and GRS 1915+105 during 1999, 2004, and 2005 - 2006 outbursts, respectively. Previously, some of these observations show an additional component, required to fit the energy spectra of SS 433 and GRS 1915+105. We carried out a detailed reanalysis for these and other BHs. We also revealed Cyg X-1, GX 339-4, and GRS 1915+105 in different spectral states and studied an evolution of X-ray energy spectra of GRS 1915+105, in detail, during the IS over shorter time intervals.

Also we analyzed the archival data of V4641 Sgr collected by RXTE and BeppoSAX (Boella et al. 1997), which were obtained in the periods of February - September, 1999 and March 13-14, 1999, respectively. Previously, these data were also analyzed, e.g., by Wilms et al. (2005); Shaposhnikov and Titarchuk (2006, 2007, 2009); Tomsick et al. (2018) (for Cyg X-1); Tomsick et al. (2009); Shaposhnikov and Titarchuk (2009) (for GX 339-4); 
Rodriguez et al. (2008); TS09; Filippova et al. (2006) (for GRS 1915+105); Miller et al. (2002), Nandi et al. (2005) (for SS 433) and in't Zand et al. (2000) (for V4641 Sgr).

Standard tasks of the LHEASOFT/FTOOLS 6.24 software package were utilized for data processing using methods recommended by the RXTE Guest observer facility according to the RXTE Cook Book. ${ }^{1}$ For our spectral analysis we used PCA Standard 2 mode data, collected in the $3-20 \mathrm{keV}$ energy range. The standard dead time correction procedure has been applied to the data. To construct broad-band spectra, we used the HEXTE data. We subtracted the background corrected in off-source observations. Only HEXTE data in the $20-100 \mathrm{keV}$ energy range were applied for our spectral analysis in order to exclude the channels with largest uncertainties. The HEXTE data have been re-normalized based on the PCA. The data are available through the GSFC public archive. ${ }^{2}$ These observations are listed in Table 1 (for Cyg X-1), Table 2 (for GX 339-4), Table 6 of TS09, Table 3 of the present paper (for GRS 1915+105), in Tables $4-6$ of ST10 (for SS 433) and Table 4 of the present paper (for V4641 Sgr). We give a special attention to observations where this additional excess in the energy spectrum was registered (TS09, ST10).

In addition we analyzed the INTEGRAL/IBIS/ISGRI spectra in the 2004 flaring stage of SS 433, coordinated with the RXTE observations. We have used the version 10.2 of the Offline Science Analysis (OSA) software distributed by the INTEGRAL Science Data Center (ISDC, http://isdc.unige.ch; see Courvoisier et al., 2003). For all considered objects we used the public data from the All-Sky Monitor (ASM) on the board of RXTE (Bradt et al. 1993) in order to obtain additional diagnostics of the source activity. The ASM light curves (in the 2-12 keV energy range) were retrieved from the public RXTE/ASM archive ${ }^{3}$.

The monitoring Ryle Radio Telescope $(15 \mathrm{GHz})$ data in the 2005 - 2006 period were kindly provided by Guy Pooley. The technical details of the radio telescope were described by Pooley \& Fender (1997). We analyzed the SS 433 spectra from the 2004 outburst which we coordinated with the simultaneous radio observations. The monitoring RATAN600 Radio Telescope $(2.3 \mathrm{GHz})$ data during the March 2 - April 12, 2004 period were available through the public archive ${ }^{4}$. We used three BeppoSAX detectors, Low-Energy and Medium-Energy Concentrator Spectrometer (LECS and MECS, respectively) and a Phoswich Detection System (PDS) detectors to analyze the V4641 Sgr data. The SAXDAS

\footnotetext{
${ }^{1}$ http://heasarc.gsfc.nasa.gov/docs/xte/recipes/cook_book.html

${ }^{2}$ http://heasarc.gsfc.nasa.gov

${ }^{3}$ HEASARC, http://xte.mit.edu/ASM_lc.html

${ }^{4}$ http://www.sao.ru/cats/satr/XB
} 
data package was utilized for performing data. We processed the spectral analysis in the good response energy range with taking into account satisfactory statistics of the source: the $0.4-4 \mathrm{keV}$ for LECS, 1.8 - $10 \mathrm{keV}$ for MECS and $15-150 \mathrm{keV}$ for PDS.

\section{Spectral and timing analysis}

To fit the broad-band energy spectra of these sources we used two different spectral models: i) an additive XSPEC model consisting of the Reflection (relxill) component (see Garcia et al. 2014) combined with diskbbody component and a XSPEC model consisting of the Comptonization (BMC or COMPTB) component (see Titarchuk et al. 1997 and Farinelli \& Titarchuk 2011 correspondingly) and the iron line (Gaussian) components. In the latter case for all spectra we also added a blackbody (BB) component, for which the bestfit color temperature was in the range of $4.5-5.9 \mathrm{keV}$. A multiplicative tbabs model takes into account absorption by neutral material. The tbabs model parameter is an equivalent hydrogen column $N_{H}$.

In Figure 1 we present our considered scenario, for the innermost part of a $\mathrm{BH}$ source. We assume that accretion onto a $\mathrm{BH}$ is described by three main zones: a geometrically thin accretion disk (e.g. the standard Shakura-Sunyaev disk, see SS73), a transition layer (TL), which is an intermediate link between the accretion disk, and a converging (bulk) region (see Titarchuk \& Fiorito 2004), that is assumed to exist, at least, below 3 Schwarzschild radii, $3 R_{S}=6 G M_{b h} / c^{2}$. Furthermore, we take into account a relatively thin layer near a $\mathrm{BH}$ horizon, where the photon-photon interaction results in the pair production (LT18). In this case the created positrons interact with accreting electrons there and therefore the annihilation line photons are possibly created and distributed over the relatively narrow shell near a BH horizon. An Earth observer can see this annihilation line only with the gravitationally redshifted energy for which the corresponding redshift, $z \gg 1$. Typically, $z \sim 20$ for the pair production region. As a result, $E_{\text {obs }} \sim E_{511} / z \sim 25 \mathrm{keV}$. Formally, this feature can be fitted by a BB XSPEC model component of the temperature $k T_{B B} \sim 4-6$ $\mathrm{keV}$.

The spectral model parameters are the equivalent hydrogen absorption column density $N_{H}$; the spectral index $\alpha$, a color temperature of the HBB, $k T_{H B B}$, normalization $\mathrm{N}_{H B B}$ of the HBB component, $\log A$ related to the Comptonized fraction $f[f=A /(1+A)]$; the color temperature and normalization of the seed photon blackbody component, $k T_{s}$ and $\mathrm{N}_{b m c}$ (or $\mathrm{N}_{\text {comp }}$ ), respectively.

We should emphasize that we use the XSPEC comptb component (see Farinelli et al. 
2007) instead of the bmc when the high energy tail is clearly presented in the data. But at energies below this exponential tail the comptb and bmc are identical.

As one can see, the $\mathrm{BMC}$ and Comptb models are the first principal models using only 4 and 5 parameters, respectively, which take into account contribution of the seed BB (two best-fit parameters: the seed photon temperature and normalization), the Comptonized component (the spectral index, in the BMC case of or the spectral index and cutoff energy in the COMPTB case and a fraction (relative normalization) of this Comptonization component. If we trace the HBB hump in the residual, we use another two best-fit parameters of this component.

For our Comptonization model, we found that the color (seed) photon temperature, $k T_{s}$ is about $1 \mathrm{keV}$ for all considered data and thus we fixed values of $k T_{s}$ at $1 \mathrm{keV}$ (see also TS09, ST10). An equivalent hydrogen absorption column density was fixed at the level of $N_{H}=6 \times 10^{21} \mathrm{~cm}^{-2}$ (Tomsick et al. 2018) for Cyg X-1, $N_{H}=5 \times 10^{21} \mathrm{~cm}^{-2}$ (Kong et al. (2002), Garcia et al. 2017) for GX 339-4, and $N_{H}=5 \times 10^{22} \mathrm{~cm}^{-2}$ (Trudolyubov 2001), $1.2 \times 10^{23} \mathrm{~cm}^{-2}$ (Filippova et al. 2006) and $2.3 \times 10^{21} \mathrm{~cm}^{-2}$ (Dickey \& Lockman 1990) for GRS $1915+105$, SS 433 and V4641 Sgr, respectively. When the parameter $\log (A) \gg 1$ we fix $\log (A)=2$ because the Comptonized illumination fraction $f=A /(1+A) \rightarrow 1$ and variation of $A$ does not improve the fit quality any more.

Similarly to the ordinary bbody XSPEC model, the BMC and Comptb normalizations is a ratio of the source (disk) luminosity L to the square of the distance $d$ :

$$
N=\left(\frac{L}{10^{39} \mathrm{erg} / \mathrm{s}}\right)\left(\frac{10 \mathrm{kpc}}{d}\right)^{2}
$$

In Tables 5-13, 15-18 we demonstrated fit qualities of our spectral model. The $R X T E$ light curves were analyzed using the powspec task from FTOOLS 6.24. The timing analysis of $R X T E / P C A$ data was performed in the $15-30 \mathrm{keV}$ energy range using the event mode for the 91701-01-49-00 observation of GRS 1915+105, where we found a noticable excess at 20 $-40 \mathrm{keV}$ in terms of our model. The time resolution for this observation is $1.5 \times 10^{-5} \mathrm{~s}$. We generated the power density spectra (PDS) in $0.1-500 \mathrm{~Hz}$ frequency range with 0.01 second time resolution. We subtracted a contribution due to Poissonian statistics and Very Large Event Window. To model PDS we used QDP/PLT plotting package.

The PDS continuum shape for BHs in the LHS and IS has usually a band-limited noise shape (Titarchuk et al. 2007), which is well presented by an KING model in QDP/PLT. To fit QPO features we use Lorentzian shape. We quote the Lorentzian centroid as a QPO frequency. 


\section{Results}

Below we present, in details, results for all studied sources using different spectral models. We compared the results using the Comptonization and reflection models and found a presence of an additional component (above $20 \mathrm{keV}$ ) in the spectra for all sources using the our Comptonization model. It is interesting that additional excess of radiation of 20 $\mathrm{keV}$ does not arise when we use the reflection model. Moreover, the Comptonization model better describes the spectral evolution of considered BHs than the reflection model. Thus below we systematically elaborated this spectral study.

We analyzed the data sets for five sources: Cyg X-1, GX 339-4, GRS 1915+105, SS433 and V4641 Sgr. Among them, for Cyg X-1, GX 339-4 we have much more extensive data than those for others. Therefore, we use these two sources for a more detailed study of the spectral transitions from the LHS to IS and from the IS to HSS. It is remarkable that three other sources (GRS 1915+105, SS433, V4641 Sgr) fit well the resulting picture of the spectral behavior.

\subsection{Cyg $\mathrm{X}-1$}

Cyg $\mathrm{X}-1$ is a classical $\mathrm{BH}$ source and one of the sources most extensively observed by RXTE (Wilms et al., 2005; ST06, ST07, ST09). Recently, a broadband spectra of Cyg X-1 applying $N u \mathrm{STAR}$ and Suzaku make it possible to improve our knowledge on the iron line spectral region of this source. Previously, these spectra of Cyg X-1 were also analyzed by Tomsick et el. (2014, 2018), Walton et al (2016) and Basak et al (2017) using so-called reflection models. They claimed their model is in a good agreement with for all spectra observed throughout spectral states of the source.

We investigate the Cyg X-1 spectra using our Comptonization model. We analyzed 832 RXTE observations of Cyg X-1 made between February 1996 and July 2005 using the public archieve (see Table 1). Using our Comptonization model we found that the source was in four spectral states: the LHS, the IS, the HSS and the VHS. We revealed a significant positive excess (so-called HBB hump, at 15-40 keV) in some Cyg X-1 spectra when we fitted the data applying the model tbabs*(comptb+gauss)*smedge. The shape of this additional component can be described by a simple bbody (HBB) model with a color temperature of $\sim 4-5 \mathrm{keV}$. As a result we use the model tbabs* (comptb+gauss+bbody)*smedge and apply it for all spectra of $\mathrm{Cyg} \mathrm{X}-1$ in order to describe the source evolution. We studied how the spectral parameters change during the state transition and also find significant HBB normalization, (see e.g. Table 5) detected mostly in the IS and sometimes in a so-called LHS- 
IS spectral transition. Cyg X-1 demonstrates a lot of spectra of this type. Furthermore, we revealed a significant HBB component even for $\Gamma<2$. But what is remarkable that the hump was observed in the range of $1.8<\Gamma<2$ and only in those cases when the electron temperature was lower $(\sim 20 \mathrm{keV}$, see Table 7 at MJD=51908 (50109-01-04-00). However, for the same index range $(1.8<\Gamma<2)$, but for higher plasma temperature $\left(k T_{e} \sim 50 \mathrm{keV}\right)$, the HBB was very weak for $\Gamma \sim 1.8$ [see 50109-03-13-00 (MJD=51919)].

We also detected a hump for the $\Gamma=2-2.5$. The hump becomes stronger when $\Gamma$ increases from 2 to 2.3 and then it is getting weaker when $\Gamma$ reaches 2.8. At high $\Gamma$, the detection of the HBB falls below the detection limit. For such a case we fix normalization of the HBB at $N_{H B B}=0.2 \mathrm{~L}_{37} \mathrm{erg} / \mathrm{s} / \mathrm{cm}^{2}$. For Cyg X-1, the necessary observational conditions for detection of the HBB are the $1.8<\Gamma<2.5$ range and relatively low electron temperatures $\left(k T_{e}\right)$. We should notice that Cyg X-1 is characterized by different levels of X-ray luminosity at different epoches and also different index values (ST09). When the luminosity level is higher, the source can achieve higher levels of the index, and vice versa (see Shaposhnikov \& Titarchuk, 2006 hereafer ST06). In Figure 2 using our Comptonization model we illustrate how Cyg X-1 evolves from the LHS (black line and observational points) to the VHS (blue line and points) through the IS (red line and points) and the HSS (green line and points), see also Table 5-8 .

The shape of this additional component can be described by the high temperature blackbody (HBB) component (Fig. 3, see upper panels). As it is seen from this Figure the maximum of the HBB component is observed when the source is in the IS. But this HBB component cannot be determined using the reflection model (see lower panels).

It is important to emphasize that the HBB hump has its centroid energy around 20 $\mathrm{keV}$ similar to that found in the Monte-Carlo simulations of the redshifted annihilation line of $511 \mathrm{keV}$ (see LT18). Specifically, we detected 169 events (among 832 ones) in which the HBB was clearly seen during the IS (see details in Tables 1, 5-8).

In Figure 4 we demonstrate the photon index $\Gamma$ vs the $R X T E$ soft X-ray flux (3-10 $\mathrm{keV}$ ) correlation for Cyg X-1 using the Comptb model (left panel) and the reflection model (right panel). One can clearly see in the left panel that $\Gamma$ evolves from values of 1.5 (in the LHS) to those of 2.2 (in the IS) with a clear signature of the index saturation at 2.2. It is worth noting another plateau at about 2.6, seen in this Figure, was found by Shaposhnikov \& Titarchuk (2006), hereafter ST06. In fact, the index plateau value depends on the plasma temperature $k T_{e}$ of the converging flow (see details in LT99 and LT11).

On the other hand one cannot see any variation of $\Gamma$ with the soft X-ray flux using the reflection model (see the right panel). The next natural question arises what typical time 
scales for the iron line-edge and the HBB are and how these features are related between each other. In Figure 5 we clearly demonstrate that the time scale of the line features is of two orders of magnitude larger of those for the HBB. These observational facts definitely indicate to the different origins of these spectral components (see more discussion in $\S 5$ ).

\subsection{GX 339-4}

GX 334-9 shows a similar X-ray behavior to Cyg X-1. The source spends most of the time in the LHS like a faint persistent source. It occasionally shows an outburst with a transition from the LHS to the HSS. Usually, during the HSS, it becomes brighter by a factor of $5-100$ in the $2-10 \mathrm{keV}$ band. In Figure 6 we show a spectral evolution of GX 334-9 from the LHS to the the very high state (VSS). The detailed analysis of the X-ray spectra in GX 339-4 is essential to identify different spectral states in this source. In addition to the LHS and the HSS, GX 339-4 also exhibits the softer spectrum and higher luminosity in VSS than that in the HSS.

Similar to the analysis of Cyg X-1 (see $\S 4.1$ ), we also applied the relxill model to the RXTE spectra of GX 339-4. We present the spectral evolution of GX 339-4 using this

reflection model in Figure 7. Best-fit results are also shown in Table ??. Applying the relxill model we found the very high iron abundance of nearly 3 and 10 times of the solar abundances (see the 92035-01-03-00 and 70109-01-07-00 observations). Although some other X-ray binaries may have supersolar abundances, it is difficult to explain why $A_{F e}$ would be variable for the same source. It is also strange that the derived binary inclination, $i$ varies from $20^{\circ}$ to $90^{\circ}$.

We continue to study the GX 339-4 spectra and analyzed them using our Comptonization model. We use 135 RXTE observations made between February 2001 and June 2007 presented in the public archive (Table 2). The best-fit results are shown in Tables $9-12$. We found 27 spectra with a significant HBB (among 135 spectra) during the IS as well as in the LHS-IS transition (see details in Tables 2, 9-12). It is interesting how the HBB evolves when GX 339-4 evolves from the LHS to the IS and vice versa (see upper panel in Fig. 7).

We also demonstrate this evolution versus $\Gamma$ and $k T_{e}$ in Figure 8. Data are taken from the RXTE observations 70110-01-97-00 ( $k T_{e}=50 \mathrm{keV}, \Gamma=1.7$, LHS, left), 90418-0101-01 $\left(k T_{e}=27 \mathrm{keV}, \Gamma=1.7\right.$, the LHS, left center), 60705-01-69-01 $\left(k T_{e}=25 \mathrm{keV}, \Gamma=1.97\right.$, LHS $\rightarrow$ IS, right center $)$, and 70110-01-08-00 $\left(k T_{e}=18 \mathrm{keV}, \Gamma=2.0, \mathrm{LHS} \rightarrow \mathrm{IS}\right.$, right $)$ Using yellow shaded areas we indicate the HBB component which presence or absence can be related to the index value, $\Gamma$ and consequently to a value of $k T_{e}$. 
When GX 339-4 is in the LHS ( $\Gamma \sim 1.7$, two left panels in Fig. 8) one does not see the HBB for $\Gamma=1.7$ for relatively high electron temperatures $\left(k T_{e}=50 \mathrm{keV}\right.$ and $k T_{e}=27$ $\mathrm{keV})$. However, when this source is in the LHS-IS transition $(\Gamma \sim 2)$, we see a significant increase of the HBB which is particularly strong when the electron temperature is lower $\left(k T_{e}=18 \mathrm{keV}\right.$, right panel) than that for the higher one $\left(k T_{e}=25 \mathrm{keV}\right)$. We found that the HBB is mostly detected in GX 339-4 spectra when $\Gamma$ changes from 1.8 to 2.5. For example, in the LHS-IS transition for $\Gamma \sim 1.85$ with a lower electron temperature $\left(k T_{e}=23 \pm 8\right.$ $\mathrm{keV}$, id=70109-01-06-00, MJD=52400.83, see Table 9) we detect a stronger HBB component $\left(N_{H B B}=4.5 \mathrm{~L}_{37} \mathrm{erg} / \mathrm{s} / \mathrm{cm}^{2}\right)$ than that for higher electron temperature $\left(k T_{e}=37 \pm 2 \mathrm{keV}\right.$, id $=60705-01-56-00, \mathrm{MJD}=52710.71$ and lower normalization, $N_{H B B}=0.2 \mathrm{~L}_{37} \mathrm{erg} / \mathrm{s} / \mathrm{cm}^{2}$, see Table 10).

In Figure 9 we show the photon index $\Gamma$ evolution versus the $3-10 \mathrm{keV}$ flux using the Comptb model. As one can see $\Gamma$ increases and then it saturates when the flux increases using the Comptb model .

\subsection{GRS $1915+105$}

Previously, we revealed the presence of the HBB spectral component in several broadband spectra of GRS 1915+105 (1996 - 2006) analyzed by TS09 where we found the HBB hump component around $20 \mathrm{keV}$ in eight IS and HSS spectra among 107 available spectra of GRS 1915+105. In the present paper we reanalyzed these data of eight observations for GRS 1915+105 (see Table 3) to check out our previous conclusions on detection or nondetection the HBB feature. It is worth noting that the HBB feature was well fitted by a $\sim 4.5 \mathrm{keV}$ BB profile (see Fig. 4 and Table 7 in TS09).

In Figure 10 we present the best-fit spectra for four different spectral states observed in GRS $1915+105$. As it is seen there luminosity increases when the source goes to the softer state. The best-fit parameters of the spectra are shown in Table 13. In Figure 11 (top panels) we demonstrate the photon index, $\Gamma$ plotted versus Comptb normalization (left) and hardness-flux diagram (right) for the same, 2005 - 2006 transition of GRS 1915+105 analyzed using tbabs*(comptb+gauss+bbody)*smedge model. From these plots we can conclude that $\Gamma$ monotonically increases with the normalization parameter (proportional to the mass accretion rate, $\dot{M}$ ) when the source makes a transition from the hard to soft state and the soft X-ray flux (or $\dot{M}$ ) grows. Furthermore, $\Gamma$ demonstrates the saturation at $\Gamma_{\text {sat }} \sim 3$. It is evident that our Comptonization model fits the source spectra for all spectral states with good statistical significance (see right column of Table 13). We should emphasize that the adopted spectral model shows a very good performance for all cases 
used in our analysis. We should note that the data related to the IS eight observations are fitted using a model, tbabs*(comptb+gauss+bbody)* smedge which is especially needed to decribe all residuals of the data ( 8 cases among 32 spectra in the present paper, and 8 cases among 107 spectra in TS09). Applying this model we found a characteristic hump around $20 \mathrm{keV}$ (see the central bottom panel of Fig. 12) which can be fitted by a BB shape of a color temperature around $4.5 \mathrm{keV}$ (HBB) (see Table 13 for values of the best-fit parameters). This HBB component is strong in each of these observations, and its EW varies from 300 to $700 \mathrm{eV}$ (see also Table 14).

Figure 13 shows the 2005 - 2006 evolutions of the flux density $S_{15 G H z}$ at $15 \mathrm{GHz}(R y l e$ Telescope, top), RXTE/ASM count rate (second from the top), the Comptb normalization, and $\Gamma$ (see two bottom panels, respectively) during the 2005 - 2006 middle and decay transitions of GRS 1915+105 (MJD 53690 - 53855). Green and red points (in two last panels) correspond to normalization and $\Gamma$. All vertical strips mark the time intervals (up to $\sim 3$ ksec), in which the HBB component was detected. For these observations, it is particularly interesting how spectral characteristics changed in the case of shorter time exposition.

Now we try to understand using as an example of GRS 1915+105 how small a lifetime of the found HBB spectral feature and thus estimate its lower limit. In Tables 14-15 we show a behavior of spectral parameters in the 10-30 s intervals (see also pink vertical strip, related to the 91701-01-49-00 observation, MJD=53771.51-53772.1 in Fig. 13). GRS 1915+105 is a bright X-ray source (not like much fainter sources SS 433 and V4641 Sgr, see below), allows to study the short time exposition spectra with a significant $\mathrm{S} / \mathrm{N}$ ratio.

The observations were carried out for the 15-30 s intervals interrupted by dead time periods. For PCA and HEXTE detectors, so called start and stop times of these short observational intervals can be different. Therefore, we applied the HEXTE time interval distribution in order to select only simultaneous (for both PCA and HEXTE) observational intervals and synchronize them to make a consistent spectral analysis. We found that the PCA spectra are well matched with the HEXTE spectra for the 91701-01-49-00 observation where the HBB hump is clearly detected. The corresponding intervals obtained for the broadband PCA\&HEXTE spectra are listed in Table 14. The HBB feature has been found only in the first eleven spectral bins of GRS 1915+105 among all available time bin intervals. For these time segments the spectra are well fitted using of the HBB component. We presented the best-fit parameters for these HBB time segments of the GRS 1915+105 observations in Tables 13-15.

We found that the color temperature of the detected HBB feature is about $4.5 \mathrm{keV}$ and equivalent width (EW) of the HBB component is in the range of $300-700 \mathrm{eV}$ for these first eleven sub-segments (about $600 \mathrm{~s}$ ) of the 91701-01-49-00 observation. As a result, this 
HBB hump is clearly detected in the spectrum of GRS $1915+105$ during first $600 \mathrm{~s}$, which can be specified as the life time of this spectral feature.

It is interesting that the observed QPO feature peaks at $\sim 1 \mathrm{~Hz}$ frequency (see details in TS09). The $\nu_{Q P O}$ presumably points out the presence of the bounded region [the transition layer (TL)] for which this QPO frequency is an intrinsic (eigen) frequency of the TL and the

power of the source, around $1 \mathrm{~Hz}$, excites this $1 \mathrm{~Hz}$ QPO. In fact, $\nu_{Q P O} \sim V_{p} / L_{t l}$ where $V_{p}$ and $L_{t l}$ are the proton velocity and the TL size, respectively (see Titarchuk et al. 1998). A characteristic value of $V_{p}$ is about $3 \times 10^{7} \mathrm{~cm} \mathrm{~s}^{-1}$ and $L_{t l} \sim 3 \times 10^{7} \mathrm{~cm}$ and thus one can obtain that $\nu_{Q P O} \sim 1 \mathrm{~Hz}$.

This QPO frequency definitely indicates to the compactness of the TL. Furthermore, GRS 1915+105 demonstrates a change of $\nu_{Q P O}$ in the PDS during the LHS-IS-HSS outburst transition in the range $0.1-10 \mathrm{~Hz}$ (Trudolyubov 2001). These lower frequency QPOs are usually observed in the IS when radio flux from GRS 1915+105 decreases to 50 mJy (see Fig. 13 here and Fig. 15 in TS09).

\subsection{SS 433 (V1343 Aql)}

Although SS 433 is located close to the "disk edge-on" position relative to the Earth observer $\left(i \sim 78^{\circ}\right)$ and it is difficult to analyze, especially in optics, nevertheless it is a source of heated debate what it is in its center-BH or NS. It is also important to study this source in the X-ray range, whose photons undergo less absorption. In addition, the object, most of its time, is in IS, which is most interesting for the purposes of our work. The fact of detecting the HBB features in the spectrum of SS433 would be a further argument in favor of a BH in this object. The X-ray spectra of SS 433 were usually fitted using the XSPEC Bremsstrahlung model for which the best-fit temperature about $20 \mathrm{keV}$ (Kawai et al. 1989; Cherepashchuk et al. 2005). However, we fitted the spectra of SS 433 using an additive Comptonization XSPEC model with a small modification of the emission line components using two narrow and wide Gaussian line components. Moreover, the data require an additional component, HBB to describe several spectra: (comptb+Gaussian1+Gaussian2+bbody)*smedge. The presence of the HBB feature has been found in 24 RXTE spectra among 117 available spectra of SS 433 (1998 - 2006), listed in Tables 4-6 in ST10. This feature has been also found in the broad-band source spectrum combined using two simultaneous observations of RXTE (ID 90401-01-01-01) and INTEGRAL (rev. 336). We should emphasize that the HBB was detected during a flare of the source.

Figure 14 illustrates (from top to bottom evolutions of the flux density $S_{2.3 G H z}$ at 2.3 
$\mathrm{GHz}$ (RATAN-600), RXTE/ASM count rate, the comptb normalization, and $\Gamma$ during the March-April, 2004 period for the rise-peak-decay transition of SS 433 (MJD 53060-53114). Vertical strips mark the observational intervals, for which an additional HBB was required. The pink vertical strip indicates the time interval of INTERGAL which was simultaneous with the RXTE observation of SS 433 (ID 90401-01-01-01). The best-fit parameters for PCA/RXTE+ISGRI/IBIS/INTEGRAL spectrum are presented in Table 16. The bestfit spectra of SS 433 shown by Seifina \& Titarchuk (2010) correspond to the combined $R X T E+$ INTEGRAL spectrum and PCA/HEXTE/RXTE spectrum. We show the photon index vs. normalization (proportional to the mass accretion rate) correlation in Fig. 11 (left bottom panel). Using this Figure one can clearly see how $\Gamma$ monotonically increases from 1.8 to 2.3 versus the mass accretion rate, $\dot{M}$ and finally saturates at high $\dot{M}$ on the level of $\Gamma \sim 2.3$. It is worth noting that in this plot we include an additional point obtained using the BeppoSAX observations of SS 433 (see $\S 2$ ).

SS 433 is a unique BH which mostly shows the IS and sometimes seen in short time transitions from the IS to LHS and vice versa. A nature of the source is highly debated. The key moment is a mass of the compact object (ranged from 1.4 to $4.2 M_{\odot}$, Gaise et al., 2004), which favors to either a $\mathrm{BH}$ or a NS cases. However, X-ray spectral signature of a $\mathrm{BH}$ as a correlation of the spectral (photon) index vs mass accretion rate developed by ST09 points to the BH nature of the central object in SS 433 (see also ST10).

\subsection{V4641 Sgr (SAX J1819.3-2525)}

Outburst events of V4641 Sgr in September 1999 are consistent with a number of well separated flares of different amplidudes depending on energy. The Wide Field Cameras (WFC) of the BeppoSAX at time MJD 51431 observed an outburst. This outburst was examined by in't Zand et al. (2000) and they found the best-fit using the XSPEC model: phabs(compTT + bbody + Gaussian). The BB temperature, $\mathrm{kT}_{B B}$ was $2.4 \pm 0.2 \mathrm{keV}$. However, Figure 7 of their paper demonstrates that the fit quality is not acceptable, mainly because of residuals around $20 \mathrm{keV}$. Moreover, the obtained color temperature of this BB component of $\sim 2.4 \mathrm{keV}$ is too high for a typical accretion disk temperature.

Therefore, we reanalyzed these X-ray spectral data. To do this new analysis we investigated the BeppoSAX NFI observation (ID=20549006) carried out on March 13-14, 1999. As we have already mentioned above, in a previous study of this spectrum, in't Zand et al. (2000) fitted the $\mathrm{Fe} \mathrm{K}_{\alpha}$ line region with two zero-width Gaussians (at $6.68 \mathrm{keV}$ and $6.97 \mathrm{keV}$ for Fe XXV and Fe XXVI), respectively with an unsuffcient fit quality. Miller at al. (2002) significantly improved the fit statistic using the Laor line model to describe the $\mathrm{Fe} \mathrm{K}_{\alpha}$ line 
region. However, all previous examinations considered the $0.4-10 \mathrm{keV}$ energy range only. In the present paper we investigated the broader band $(0.3-150 \mathrm{keV})$ spectrum of V4641 $\mathrm{Sgr}$, adopting the Comptb model for the continuum and the Laor line model for $\mathrm{Fe} \mathrm{K}_{\alpha}$ line region.

We used the RXTE data for V4641 Sgr (see Table 18, first column for obs. IDs ) and analyzed the data similarly to that for GRS $1915+105$ and SS 433 (see upper panels in Figure 15). However, the RXTE detectors cannot provide high quality spectra for energies below $3 \mathrm{keV}$ while a broad energy range of BeppoSAX allows us to reliably determine the Comptb parameters using soft energies. Due to a good spectral resolution of BeppoSAX NFI detectors one can find the sharp K-edge at $7 \mathrm{keV}$ in the spectrum of V4641 Sgr. In addition, we revealed the HBB component with a color temperature of about $4.5 \mathrm{keV}$. The best-fit parameter values are shown in Tables 17-18 while in Fig. 15 we show the corresponding best-fit spectra of V4641 Sgr during the IS event in $\mathrm{E}^{*} \mathrm{~F}(\mathrm{E})$ units. The data are presented by crosses using the spectral model: tbabs*(comptb+laor+bbody)*smedge. The spectral model components are shown for the Comptb, Laor, and BB components (see details of the fits in Tables 17-18).

The determination of the HBB hump can be affected by the presence of nearby Feline K-complex $(6.5-7 \mathrm{keV})$ associated with $\mathrm{K}$-edge at $\sim 7 \mathrm{keV}$. However, the photoelectric absorption cross section sharply decreases with a photon energy [approximately $\left.\sigma_{p h} \sim(7.8 \mathrm{keV} / E)^{3} \sigma_{T}\right]$, and therefore K-edge cannot change a central part $(10-30 \mathrm{keV})$ of the HBB hump. This is clearly seen in Fig. 15 (lower panel) where the BeppoSAX NFI spectrum (ID=205490061) of V4641 Sgr obtained with a high energy resolution was fitted by our Comptonization model.

As we have already mentioned above this V4641 Sgr spectrum was investigated in detail in the previous studies by in't Zand et al. (2000) and Miller et al. (2002). But their studies were based on the soft spectrum $(0.4-10 \mathrm{keV})$ and therefore their results differ in values of the photon index $\Gamma$. For example, In't Zand et al. claimed that they found the high soft state spectrum of V4641 Sgr dominated by the disk component with a weak power law of $\Gamma \sim 3$. They also found the absence of narrow or broad-band features in the PDS of MECS light curve. On the other hand, Miller et al. argued about an IS identification, based on the obtained value of the photon index, $\Gamma=1.3 \pm 0.1$ and intermediate X-ray luminosity. Because of the low value of $\Gamma$, Miller et al. suggested that V4641 Sgr was observed in a unique substate of the IS, which may differ from similar states in other Galactic BHs. However, we studied a broad-band spectrum $(0.3-150 \mathrm{keV})$ of V4641 Sgr and found a value of the photon index $\Gamma=2.5 \pm 0.1$, which is in agreement with a typical IS identification (see Table 17). In the radio observations of V4641 Sgr a double-sided jet structure has been detected 
in VLA image taken on 16 September, 1999 (Hjellming et al. 1999). These events could indicate to a radio active state like to GRS $1915+105$ and SS 433 during X-ray outbursts.

Now, we can summarize the main spectral parameters for all five objects that there is a common condition for the detection the HBB feature. All these spectra where we revealed these HBB components, are characterized by the photon index of the Comptonized component, $\Gamma_{\sim}^{>} 2$ (compare with LT18). It is also important that we have found using the $R X T E$ data for Cyg X-1 that the time scale of the iron line features is much longer (of order of $10^{5} \mathrm{~s}$ ) than that for the HBB component (of order of $10^{3} \mathrm{~s}$ ) and which indicate that these two components are unrelated and formed in different parts of the source.

\section{Interpretation and discussion of observational results}

Before to proceed with an interpretation of the analyzed observations let us briefly summarize them as follows:

i. We detected the HBB hump in five black holes in their intermediate state (IS) and the LHS-IS, IS-HSS transitions. ii. The HBB hump was found in the IS spectra and related to X-ray flux of order of $10^{37} \mathrm{erg} / \mathrm{s}$. iii. A strong iron-line complex is seen along with the HBB broad feature and they are unrelated in terms of time scales. iv. Usually, the IS spectra are accompanied by a moderate radio emission. v. Our model in which the continuum is characterized by only 5 parameters and therefore has high significance. vi. The meaning of the normalization in our model is related to the disk seed photon flux and it is proportional to the disk mass accretion rate.

It is interesting that the HBB hump was detected previously, for example, in the Cyg X1 spectra using Suzaku + NuSTAR data (e.g., see Fig. 2 in Walton et al. 2006; Figs. 4 and 5 in Tomsick et al. 2018). However, this hump was interpreted as a reflection bump using the diskbb + reflionx model. But it is well known that the reflection (downscattering) is uneffective when $\Gamma>2$ (or around 2) because of lack of high energy photons. Thus the reflection hump is not formed in these cases (see LT07).

The loss of the HBB hump detection is usually caused by averaging spectra of different spectral states (see ST09). Moreover, this feature shortly lives and extremely transient. The nature of the HBB component (or so called a reflection hump in the literature) is still hot

disputable. So, it is reasonable to consider possible interpretations and thoroughly study the observational manifestations of this feature along outburst rise/decay in detail. 


\subsection{Observations of the HBB component}

Using spectral analysis of BHs: Cyg X-1, GX 339-4, GRS 1915+105, SS 433, V4641 Sgr observed by RXTE, INTEGRAL and BeppoSAX we found that HBB component is well detected in the IS spectra. Also this HBB is never detected in the LHS spectra. We find some detections of the HBB in the LHS-IS and the IS-HSS spectra when $\Gamma$ changes from 1.8 to 2.8 and $k T_{e}$ is around $20 \mathrm{keV}$ or less (see e.g. Cyg X-1 and GX 339-4 cases). We also detected this $\mathrm{HBB}$ of the color temperature of $4.5-5 \mathrm{keV}$ in the energy spectrum of GRS 1915+105 (see Figs. 12-13).

However, it is important to know if this HBB feature presents through the whole observation or not. We divided the particular PCA and HEXTE simultaneous observation into subintervals and find that the lifetime of this feature is about of $11 \mathrm{~min}$ in the case of GRS 1915+105 (see also Table 14). But it was absent for following observations (12 - 32, Table 14). Although the total duration of this observation is around 30 minutes but the HBB was seen only during the first 10 minutes.

The HBB feature is well detected in the energy spectrum, when a BH:

- is in the active phase, namely, in the IS or during the IS-LHS and IS-HSS transitions;

- is characterized by a value of dimensionless mass accretion rate, $\dot{m} \sim 1$ (see LT18);

- is accompanied by moderate radio-wave emission (see Figures 13-14);

- is characterized by a photon index in the range of $1.8<\Gamma<2.8$ (see Fig. 16);

- is observed when X-ray luminosity in the range of $5 \times 10^{36}-5 \times 10^{37} \mathrm{erg} / \mathrm{s}$;

On the other hand we associate unfavorable conditions with the LHS and HSS, when the HBB is usually absent or smeared out (see, as an example, Fig 12) We hope that the current and future observations can easily detect this HBB feature based on our empirical criteria. There are many examples of the detection of such a hump-like feature in the X-ray source spectra by other authors. For example, Walton et al. (2016) [Fig. 9 there] clearly demonstrate the presence of the hump in the energy band from 10 to $40 \mathrm{keV}$ in the Cyg X-1 spectrum using Nustar and Suzaku data. Another example of the observation of the high/soft spectrum of Cyg X-1 is shown by Tomsick et al (2014). The authors also find the high/soft spectra (see Fig. 5, panel a there) where one can see a hump between 10 and $40 \mathrm{keV}$. According to LT07 (see also ST80) the downscattering (reflection) hump should be clearly observed in the hard state demonstrated by Garcia et

al. We emphasize that the strongest effect of the down-scattering should be observed in the LHS. But if the down-scattering hump is not formed in the LHS then it should not be formed 
in the reflection case too. In other words in the reflection case a fraction of the reflected (down-scattered) photons, $25 \%$ is much less than that for the transmission case when the fraction of the down-scattered photons is $100 \%$.

\subsection{Similarity between the upscattering photon spectra and the distribution of capital gain due to investement}

Titarchuk et al. (2009), hereafter TLS09, strongly argue that there is a full similarity between the formation of the Comptonization (upscattering) photon spectrum and capital gain due to investment [see also Dragulescu \& Yakovenko (2001)]. Furthermore, the photon upscatering is analogous to the Fermi particle acceleration. TLS09 derive the Comptonization spectrum as a broken power-law (see Eq. A5, there) for which the spectral index of the hard tail $\alpha$ is

$$
\alpha=\frac{\ln (1 / p)}{\ln (1+\eta)}
$$

where $p$ is average probability of photon scattering in the CC, or in the case of capital gain is average probability of success due to investment and $\eta$ is related to the average photon energy (money) change per scattering (investment) $\Delta E=\eta E$ (where $\eta>0$ for the upscattering case or successful investment). TLS09 demonstrated that $\alpha \approx\left(\eta N_{s c}\right)^{-1}=Y^{-1}$ where $Y$ is the Comptonization parameter [see Rybicki \& Lightman (1979)].

Thus one can suggest the bounded transition layer [Compton cloud (CC)] near the central object, a NS or a $\mathrm{BH}$ where the well-known Comptonization spectrum is produced (see e.g. ST80). Titarchuk \& Seifina (2019) argue this bounded configuration (CC) is formed due to an adjustment of the Keplerian disk motion to the inner sub-Keplerian rotation of the central object. The observational change of the photon index due to the mass accretion rate is well established (see Fig. 11 and ST09). But the relative constancy of the index in the framework of the reflection model (see Fig. 4, right panel) can be only explained by unnecessary surplus of free parameters using this model. Moreover, applying the reflection model we cannot see a well-known the spectral transition in BHs (see e.g. Park et al. 2004 and ST09).

The main portion of the X-ray spectrum can be described and explained by a Comptonization of the soft (disk) photons, parametrized by a photon index $\Gamma$. In its turn, $\Gamma$ inversely proportional to the Comptonization parameter $\mathrm{Y}$ is a product of upscaterring efficiency at single scattering $\eta$ and number of scattering $N_{s c}$ (see Eq. 2 and text below of it). Thus the spectral index evolution indicates to a different spectral state, and is essential to constrain spectral models (see also McCintock 2005; Remilard \& McClintok 2006). 
In other words if the spectral model does not reproduce the spectral evolution (in terms of its parameters) and by time evolution (LF-QPO frequency vs soft X-ray luminosity or mass accretion rate), then something is wrong with this particular model

In addition, the Comptonization model (Comptb, Comptt, BMC) is based on a transitionlayer paradigm (TLM98), in which the LF-QPO self-consistently reproduces their frequency, and the correlation $\Gamma$ versus LF-QPO frequency during transitions between diffent spectral states (e.g., see Vignarca et al., 2005; Shaposhnikov \& Titarchuk, 2009; Stiele et al. 2010).

It turns out that this Comptonization model is universal and applicable to any other situation (like to the upscattering photon spectra and the distribution of capital gain due to investement) where the similar nature of processes occurs.

\subsection{A possible origin of the HBB Component}

We find an observational evidence of the $\mathrm{HBB}$ around $20 \mathrm{keV}$ which can be fitted by a $\sim 4.5 \mathrm{keV}$ blackbody profile [see for example, Figures 3, 7, 8, 12, 15 and Tables 13-18]. One can argue that this observable hump at $20 \mathrm{keV}$ is a signature of the Compton reflection hump. The reflection or downscattering interpretation encounters a principal difficulty because the hard power-law tails of these spectra are too steep to form the Compton hump. (see LT07). As it is seen from Tables 5-8, 9- 18 that in almost all spectra where we found a noticable HBB the photon index $\Gamma>2$.

One can concern regarding a way how the current (reflection) paradigm is excluded as the origin of the hump. As we have already emphasize in the Intoduction that LT07 demonstrate that the characteristic downscattering hump is only formed when the spectral index, $\alpha<1$ (or $\Gamma<2$ ). It can be another counter argument against our interpretation of the hump that we use too simple models for fitting of the data not like in more recent modeling [see e.g. the relxill (reflionx) model] and the comparison with these more complex models (commonly used by the community in the context of the current paradigm). Now it becomes clear using our detailed investigations (see Fig. 5) that the iron line component used in the reflection model is not related to the HBB because they have very different time scales (at least two order difference in the time scales) and thus this modelling is not related to the real observations.

Specifically, Figure 5 demonstrates time-scale characteristics for HBB-feature (red) and Fe-Gaussian line (blue). Here we assume that if this (iron-Gaussian) line is seen continuously in some observations (with approximately the same flux or parameters $N_{\text {line }}, E_{\text {line }}$ ), then this line is likely to remain visible in the source spectrum between these observations. As can 
be seen from our Tables, these interphases are smaller than the intervals of the observations themselves. In addition, we divided the observational interval ( $\sim 3 \mathrm{ks})$ into shorter subintervals. Thus we checked that the line is constant during the entire observation, i.e. it does not disappear and is not variable within this observational interval Then we summed up the times of all these intervals during a continuous sequence of observations, when this iron-Gaussian line is detected. But for the HBB-feature case, we found observations (3 ks each), in which the HBB-feature is detected (see Table 3), and then we divide the entire observational interval into sub-intervals of 10-15 seconds, this HBB feature is not detected in some sub-intervals (Table 15). Then we chose a continuous chain of sub-intervals, in which the HBB-feature is confidently detected and not detected before and after this chain (we assume that HBB-feature is detected when its $E W \geq 70 \mathrm{eV}$ ). Furthermore, we summed up the times of these sub-intervals (10-15 seconds each). Thus we estimated the characteristic lifetime of the HBB-feature. In both cases (iron-Gaussian line and HBB feature), we checked the stability of our result in a sample of all available observations.

As a result, we demonstrate the characteristic lifetime of the iron-Gaussian line in Figure 5 by red line against normalization, where the total time during which the ironGaussian line continuously observed is pointed out on the vertical axis. At the same time, along the horizontal axis, we indicated normalizations $\left(N_{\text {line }}\right)$ with which the iron-Gaussian line was observed. The blue points show HBB-feature detection: the vertical axis indicates the total time during which the HBB-feature was continuously observed (sometimes it was $3 \mathrm{ks}$, i.e. one observation, $800 \mathrm{~s}$, i.e. less than the whole observations, and sometimes several successive observations). At the same time, along the horizontal axis, we indicate normalizations $\left(N_{H B B}\right)$ with which the HBB-feature was observed (Table 15). This Figure is given for $\mathrm{Cyg} \mathrm{X}-1$, as an example, in which we wanted to show that the times of continuous visibility (we called this lifetime) of each of the features are very different. In the vertical axis, where the visibility time is set, the lifetime for the HBB-feature is $10^{3}-10^{4} \mathrm{~s}$, while the lifetime for the Fe-line is $\geq 10^{5} \mathrm{~s}$. In this Figure, we demonstrated that these spectral details are formed in geometrically different areas of the source. Indeed, if HBB-feature and iron-line have different lifetimes, then most likely they are formed in different places of the source. Moreover, the HBB-feature is in a more compact area, i.e. closer to a BH, while the Fe-line is located in a wider area, i.e. farther from a BH. And since they are formed in completely different places, then most likely they have different conditions of formation and different origins.

We describe the HBB hump by a blackbody shape. This model has only two parameters: the color temperature and normalization. The equivalent width (EW) of the hump should be independent of the iron and other heavy elements, ionization parameter, because this hump is always distributed between 10 and $40 \mathrm{keV}$ (see e.g. Fig. 15) where there is no any of these 
lines. We should also emphasize that our spectral model tbabs*(comptb+bbody)*smedge plus one or two Gaussians has only five free parameters. It is worth noting that we use seven parameters when we need to use two more parameters for the bbody component (HBB). One can advice us to include the absorption edge in our best-fit models for RXTE, INTEGRAL, BeppoSAX data. But these data do not require any additional absorption components (see values of $\chi_{r e d}^{2}$ of the best-fits using our models in Tables 5-8, 9-18.

Evolutions of spectral characteristics of Cyg X-1, GX 339-4, GRS 1915+105, SS 433 and V4641 Sgr are very similar. While they have different BH masses all of them reveal the HBB feature which is centered around $20 \mathrm{keV}$. It is interesting that the HBB feature is usually observed in the same X-ray luminosity range $\left(5 \times 10^{36}-5 \times 10^{37} \mathrm{erg} / \mathrm{s}\right)$ for different sources.

One can raise a natural question on the origin of the HBB. LT18 estimated the optical depth for photon-photon interaction very close to a BH horizon. In order to do this they calculated the photon density $N_{\gamma}$ near a black hole horizon, assuming that the most photons there have energy greater than $m_{e} c^{2}=511 \mathrm{keV}$ :

$$
N_{\gamma}=\frac{L_{\gamma}}{4 \pi r^{2} c m_{e} c^{2}},
$$

where $L_{\gamma} \simeq 10^{37}\left(M_{b h} / 10 M_{\odot}\right) \mathrm{erg} / \mathrm{s}, r=R_{\text {sch }}=2 G M / c^{2}$ is the Schwarzschild radius, $R_{\text {sch }}=$ $3 \times 10^{6}\left(M_{b h} / 10 M_{\odot}\right) \mathrm{cm}, c$ is the speed of light $\left(3 \times 10^{10} \mathrm{~cm} / \mathrm{s}\right)$ and the electron rest energy, $m_{e} c^{2}$ is about $5 \times 10^{-7} \mathrm{erg}$. As a result, LT18 obtained that $N_{\gamma}=0.6 \times 10^{19} /\left(M_{b h} / 10 M_{\odot}\right)$ $\mathrm{cm}^{-3}$.

Then, LT18 estimate the optical depth for photon-photon interactions as

$$
\tau_{\gamma-\gamma} \sim \sigma_{\gamma-\gamma} N_{\gamma} R_{s c h} .
$$

The cross section for $\gamma-\gamma$ interaction, $\sigma_{\gamma-\gamma} \sim 0.2 \sigma_{T}$, where $\sigma_{T}=6 \times 10^{-25} \mathrm{~cm}^{2}$ is the Thomson's cross-section. As a result one can find that $\tau_{\gamma-\gamma} \sim(1-2)$ using Eqs. (3-4). It is worth noting that $\tau_{\gamma-\gamma}$ is independent of a $\mathrm{BH}$ mass.

As one can see the IS luminosity provides the sufficient $\tau_{\gamma-\gamma}$ for the photon-photon interaction very close to a $\mathrm{BH}$ horizon. On the other hand, pairs (electrons and positrons) are effectively generated as a result of $\gamma-\gamma$ interaction near a $\mathrm{BH}$ horizon. Some of the generated positrons propogate ourwards and in the way out they interacts with accreting electrons leading to the formation of the annihilation line, $511 \mathrm{keV}$. A significant fraction of these line photons can directly escape to the Earth observer if the Klein-Nishina optical depth, $\tau_{\mathrm{KN}}$ at $511 \mathrm{keV}$ is of order of one. In the way out these $511 \mathrm{keV}$ line photons undergo gravititational redshift with $z$ around 20 forming the HBB of the color temperature of around 
$5 \mathrm{keV}$. Observationally this HBB feature has an equivalent width in the interval from 400 to $800 \mathrm{eV}$ (see Fig. 16). However, if the resulting X-ray luminosities $\left[L_{\gamma} \gg 10^{37}\left(M_{b h} / 10 M_{\odot}\right)\right.$ $\mathrm{erg} / \mathrm{s}$ ], then the dimensionless mass accretion rate is much greater than 1 (see Titarchuk \& Zannias 1998) and pairs along with the $511 \mathrm{keV}$ annihilitation photons generated near a BH horizon cannot escape because they are effectively scattered off converging electrons. This our conclusion is firmly confirmed by the Monte Carlo simulations (see LT18). It is not by chance that we cannot see the HBB hump in the HSS. In the LHS we cannot see the HBB and consequently the redshifted annihilation line either because $\tau_{\gamma-\gamma} \ll 1$ and pairs are not effectively generated and the converging flow is surrounded by the hot, relatively thick Compton Cloud (CC) of the plasma temperature of order of 50-60 keV. The escaping HBB photons are scattered off hot electrons of the CC.

\section{Discussion}

One can ask various questions regarding the content of the presented paper. For example, if he/she takes a simple exponentially cutoff power-law, some of which comes directly to the Earth observer and some of which shines on a cold disk and then comes to the Earth, what does that look like? In fact, this is an interesting question. If the index of this exponentially cutoff power-law is too large then it should have high residuals at low-energies which is obvious. This is well-known effect of this simplification. As for the comparisons to standard reflection models one should be very careful to use it, because of two reasons: $i$. there is no efficiency of the reflection for the spectral index $\alpha$ higher than 1 (or the photon index $\Gamma>2$ higher than 2). In this case there is no a so called reflection hump. This is a very strong theorem of the radiative transfer (see this proof in Laurent \& Titarchuk 2007). ii. Another one is that the disk illumination fraction of the photon emitted by the central object (COMPTON cloud) should be only about 25\% (see details in Lapidus et al. 1985).

The next question is about an observation of a reflection hump. Do we expect to see feature like those in the relxil code (i.e., a fluorescent Fe line and associated Fe edge, fluorescent emission and edges at lower energies, an overall reduction in photon energy, whether this manifests as changes in powerlaw slope and cutoff energy, or also produces a hump for given photon indices? In fact, we use the Gaussian model to describe a fluorescent Fe line and associated Fe edge in an additive manner since it is obvious that the iron line and its profile can be formed in different places of the source. In Figure 5 we demonstrate that there are drastically different time scales (at least two order of magnitude difference) between Fe emission line and HBB component. This is a very important observational result!

Does the answer that the relxil code provides us to the above questions are fundamentally 
wrong? Or is the relxil code plausibly correct in the spectral calculation that it performs? The Relxill model describes both the continuum and emission features, without attracting additive components. But for the final description of the spectra "in the relxill model", power and blackbody components are still involved, the interaction with which is not specified in terms of physical parameters. But what we have revealed that the different components of the spectra of a source coming to the Earth are formed in different parts of the source. At this stage using the Relxill model we are faced with a problem of the constancy of the photon index when the spectral state changes, which is impossible in terms of the spectral evolution established by many authors using a huge set of BHs (see the Introduction). Usually in BHs the evolution of the photon index from 1.4 (LHS) through 2 (IS) to 3 (HSS) is observed.

For example, Tomsick et al. (2018), in the end, advocate for a more complex model in Cyg X-1 that there is a directly viewed powerlaw, in addition to the reflected powerlaw. But the reflection is relativistically smeared in terms of Tomsick et al.. Although this model has now become a much more complex model, it is not necessarily unrealistic, since we know from Chandra-HETG data that there is a narrow component to the Fe line, likely coming from much further away from the innner accretion flow region because Fe-line formed in the inner-most parts of the accretion flow could not survive. Moreover this emission occurred there to be distorted due to a coronal wind (see Laming \& Titarchuk 2004, Laurent \& Titarchuk 2007). It is much easier to allow the formation of iron lines in the outer parts of the disk or in the wind. One can see the confirmation of this line formation in the extended part of the source (see Fig. 5). Thus the most simple version of the reflection models are not particularly good descriptions of the data, as we show here.

One can say that our model still has issues that need to be addressed as a self-consistent description of the iron line and smedge components. However, Laming \& Titarchuk (2004) and Laurent \& Titarchuk (2007) use the self-consistent models to describe the iron-line and smedge components. Also one should keep in mind that the reflection effect does not take place if the spectral index $\alpha$ (or the photon index $\Gamma$ ) are very close to 1 (or 2 ) correspondingly. Moreover, if $\Gamma>2$ for which we found a lot of data the reflection does not work at all while in the observations we see the HBB hump for these values of the photon index $\Gamma$.

\section{Conclusions}

In this paper we demonstrated the HBB feature in the observed spectra of five Galactical $\mathrm{BHs}$ and suggest a scenario which allows us to understand how this spectral feature is formed in a BH (see more details in LT18). This HBB feature is usually observed in the IS which is characterized by suitable conditions for the HBB observations, due to the low Klein-Nishina 
optical depth at sufficiently high mass accretion rate of order $1-2$, see LT18. In the IS $\Gamma_{\sim}^{>} 2$ and thus accordingly to LT07 the HBB hump cannot be due to the reflection effect. But in the LHS $\left(\Gamma_{\sim}^{<1.5)}\right.$, this hump hump can be a result of the downcattering in an outflow (or reflection from the surrounding disk).

Previously, we reported a detection of the HBB component in the energy spectra around $20 \mathrm{keV}$ in the GBHs, GRS 1915+105 and SS 433 (see TS09, ST10). Now, we have presented a further study of this spectral feature using five galactic BHCs: Cyg X-1, GX 339-4, GRS 1915+105, SS 433 and V4641 Sgr using our spectral models in which Comptonization effects are taken into account. In particular, we have studied an evolution of X-ray energy spectra with a short time step along the IS of GRS $1915+105$ as a sample of a bright X-ray source. It was shown that the best-fit spectra obtained applying a short time exposition (10 - $30 \mathrm{sec}$ ) indicate to the HBB around $20 \mathrm{keV}$.

We detected the prominent HBB bump in Cyg X-1 and GX 339-4 mainly in their IS and sometimes in LHS-IS $(1.8 \leq \Gamma \leq 2)$ and IS-HSS $(2 \leq \Gamma \leq 2.5)$ states, when the electron temperature is relatively low (see LT99, LT11, LT18). The HBB hump increases when a BH approaches to a classical IS $(\Gamma \sim 2.3)$. This conclusion is based on extensive investigations of all aforementioned BHCs.

We also presented spectral analysis of the INTEGRAL+RXTE observation which revealed the same HBB feature in SS 433. Furthermore, we detected this HBB in another Galactic BH, V4641 Sgr using the RXTE and BeppoSAX NFI detectors. We showed that the broadband energy spectra of Cyg X-1, GX 339-4, GRS 1915+105, SS 433 and V4641 Sgr during all observations could be adequately presented by a sum of the Comptonization (BMC or COMPTB) component, the iron (Gaussian) line. But in quite a few observations of the IS observations we should add the HBB component of the color temperature $\sim 4.5-5.9 \mathrm{keV}$ to fit the data

We should also note that all these spectra with the HBB component are observed during the moderate radio active phase. Our observational results on the HBB hump suggest that we observe the gravitationally redshifted annihilation line emission in these source (see details in LT18). The detection of the HBB hump as a redshifted annihilation line emission is possibly a new observational evidence for the presence of a $\mathrm{BH}$ in these binaries.

The detection of the redshifted annihillation humps (which energy $E_{\text {anh }}=511 \mathrm{keV}$ in the comoving frame) in $\mathrm{BH}$ spectra at the energy from 10 to $40 \mathrm{keV}$ in the the lab frame is a sensitive probe of the gravitational field in the Galactic black holes (Cyg X-1, GX 339-4, GRS 1915+105, SS 433, and V4641 Sgr). This spectral feature is formed near the event horizon radius (at $R_{S}$ ) and thus this detection is a direct probe of the General Relativity 
effect.

We acknowledge the interesting remarks and points of the referee. ES thanks the IT support team (CNRS, LAPTh and LAPP, Annecy, France) for providing computing facilities. ES also thanks the LAPTh, Annecy (France) for hospitality and financial support of this work. 


\section{REFERENCES}

Ballantyne, D. R., Ross, R. R., \& Fabian, A. C. 2001, MNRAS, 327, 10

Basak, R. \& Zdziarski, A.A. 2016, MNRAS, 458, 2199

Basko, M. M., Sunyaev \& Titarchuk, L. G. 1974, A\&A, 31, 249

Boella, G. et al. 1997, A\&AS, 122, 327

Bradt, H.V., Rothschild, R.E. \& Swank, J.H. 1993, A\&AS, 97, 355

Courvoisier et al. 2003, A\&A, 411, L53

Chakrabarti, S. K., \& Titarchuk, L. 1995, ApJ, 455, 623 (CT95)

Cherepashchuk A. M., et al. 2005, A\&A, 437, 561

Dauser, T., García, J., Wilms, J., et al. 2013, MNRAS, 430, 1694

Dauser, T., Svoboda, J., Schartel, N., et al. 2012, MNRAS, 422, 1914

Dove, J. B., Wilms, J., Maisack, M., \& Begelman, M. C. 1997, ApJ, 487, 759

Dickey, J.M., \& Lockman, F.J. 1990, ARAA, 28, 215

Dovčiak, M., Karas, V., \& Yaqoob, T. 2004, ApJS, 153, 205

Dragulescu, A., \& Yakovenko, V. M. 2001, Physica. A, 229, 213

Dumont, A.-M., Collin, S., Paletou, F., et al. 2003, A\&A, 407, 13

Dumont, A.-M., Czerny, B., Collin, S., \& Zycki, P. T. 2002, A\&A, 387, 63

Fabian, A. C., \& Vaughan, S. 2003, MNRAS, 340, L28

Fabian, A. C., Iwasawa, K., Reynolds, C. S., \& Young, A. J. 2000, PASP, 112, 1145

Farinelli, R. \& Titarchuk, L. 2011, A\&A, 525, 102

Farinelli, R., Titarchuk, L. \& Frontera, F. 2007, ApJ, 662, 1167

Fender, R.P. \& Belloni, T. 2004, ARA\&A, 42, 317 (FB04)

Fender, R.P. 2001, MNRAS, 322, 31

Filippova, E., Revnivtsev, M. Fabrika, S. et al. 2006, A\&A, 460, 125 
Furst et al. 2015, ApJ, 808, 102

García, J., Harrison, F., Tomsick, J. et al. 2017, ATel 10852

García, J., Dauser, T., Lohfink, A. et al. 2014, ApJ, 782, 76

García, J., Dauser, T., Reynolds, T. R. et al. 2013, ApJ, 768, 146

García, J., \& Kallman, T. R. 2010, ApJ, 718, 695

García, J., Kallman, T. R., \& Mushotzky, R. F. 2011, ApJ, 731, 131

George, I. M., \& Fabian, A. C. 1991, MNRAS, 249, 352

Goranskijn V.P. et al., 1998, Astron. Rep., 42, 209; ibid., p.336

Greiner, J., Cubu, J.G., McCaughrean, M. J., Castro-Tirado, A. J. \& Minnickent, R. E. 2001, A\&A, 373, L37

Guilbert, P. W., \& Rees, M. J. 1988, MNRAS, 233, 475

Haardt, F. 1993, ApJ, 413, 680

Harlaftis, E.T. \& Greiner, J. 2004, A\&A, 414, L13

Hillwig, T. C. \& Gies, D. R. 2008, ApJ, 676, L37

Hjellming, R.M., Rupen, M.P. \& Mioduszewski, A.J. 1999, IAUC 7265

In’t Zand, J. J. M, Kuulkers, E., Bazzano, A., Cornelisse, R., Cocchi, M., Hiese, J., Miller, J. M., Natalucci, L., Smith, M. J. S \& Ubertini, P. 2000, A\&A , 357, 526

Koljonen, K. I. I., McCollough, M. L., Hannikainen, D. C. \& Droulans, R. 2013, MNRAS, 429,1173

Kong, A. K. H., Charles, P. A., Kuulker, E. \& Kutamoto, S. 2002, MNRAS, 329, 588

Kylafis, N. D. \& Belloni, T. 2015, A\&A, 574, A133

Laming, M. \& Titarchuk, L. 2004, ApJL, 615, L121

Laor, A. 1991, ApJ, 376, 90

Lapidus, I.I., Syunyaev, R.A. \& Titarchuk, L. 1985, Ap., 23, 663

Laurent, P. \& Titarchuk, L. 2018, ApJ, in press (see also arXiv:1804.08146) (LT18) 
Laurent, P. \& Titarchuk, L. 2011, ApJ, 727, 34 (LT11)

Laurent, P. \& Titarchuk, L. 2007, ApJ, 656, 1056 (LT07)

Laurent, P. \& Titarchuk, L. 1999, ApJ, 511, 289 (LT99)

Lightman, A. P., \& White, T. R. 1988, ApJ, 335, 57

Lightman, A. P., Lamb, D. Q., \& Rybicki, G. B. 1981, ApJ, 248, 738

Lightman, A. P., \& Rybicki, G. B. 1980, ApJ, 236, 928

Ludlam et al. 2017a, ApJ, 836, 140L

Ludlam et al. 2017b, ApJ, 838, 79L

McClintock, J., \& Remillard, R. 2003, in Compact Stellar X-Ray Sources, ed. W. H. G. Lewin \& M. van der Klis (Cambridge: Cambridge Univ. Press), preprint (astro-ph/0306213)

McClintock, J. E., Remillard, R. A., Rupen, M. P., et al. 2007, arXiv:0705.1034

Magdziarz P. \& Zdziarski A. A. 1995, MNRAS, 273, 837

Makishima K., et al. ApJ, 1986, 308, 635

Matt, G., Perola, G. C., \& Piro, L. 1991, A\&A, 247, 25

McClintock et al. 2014, SSRv, 183, 295

McClintock et al. 2009, arXiv:0705.1034

Memola E., Fendt, Ch. \& Brinkman, 2002, A\&A, 385, 1089

Miller, L., Turner, T. J., \& Reeves, J. N. 2008, A\&A, 483, 437

Miller, J. M., Fabian, A. C., in't Zand, J. J. M., Reynolds, C. S., Wijnands, R., Nowak, M. A. \& Lewin, W. H. G. 2002, ApJ, 677, L15

Mineo T. et al. 2012, A\&A, 537, 18

Mitsuda K., et al. 1984, PASJ, 36,741

Morrison, R. \& McCammon, D. 1983, ApJ, 270, 119

Nandi, A., Chakrabarti, S.K., Belloni T. \& Goldoni, P. 2005, MNRAS, 359, 629

Nayakshin, S., \& Kallman, T. R. 2001, ApJ, 546, 406 
Nayakshin, S., Kazanas, D., \& Kallman, T. R. 2000, ApJ, 537, 833

Orosz J., Kuulkers E., van der Klis M., et al. 2001, ApJ, 555, 489

Park, S.Q. et al. 2004, ApJ, 610, 378

Parker et al. 2016 ApJ, 821, L6

Parker et al. 2015 ApJ, 808, 9

Pooley G., \& Fender R. 1997, MNRAS 292, 925

Reynolds, C. S., Brenneman, L. W., Lohfink, A. M., et al. 2012, ApJ, 755, 88

Reynolds, C. S., \& Nowak, M. A. 2003, PhR, 377, 389

Revnivtsev, M. et al. 2002, A\&A 391, 1014

Rodriguez J., Hannikainen D.C., Shaw S.E. et al. 2008, ApJ, 676, 1436

Romney, I. D., Schilizzi, R. T., Fejet, I., Spencer, R. E. 1987, ApJ, 321, 822

Ròżaǹska, A., \& Madej, J. 2008, MNRAS, 386, 1872

Ròżaǹska, A., Dumont, A.-M., Czerny, B., \& Collin, S. 2002, MNRAS, 332, 799

Ròżà̀ska, A., \& Czerny, B. 1996, AcA, 46, 233

Ross, R. R., \& Fabian, A. C. 2007, MNRAS, 381, 1697

Ross, R. R. 1979, ApJ, 233, 334

Ross, R. R., Weaver, R., \& McCray, R. 1978, ApJ, 219, 292

Ross, R. R., \& Fabian, A. C. 1993, MNRAS, 261, 74

Ross, R. R., \& Fabian, A. C. 2005, MNRAS, 358, 211

Seifina, E. Titarchuk, L. \& Shaposhnikov, N. 2014, ApJ, 789, 57

Seifina, E. \& Titarchuk, L. 2010, ApJ, 722, 586 (ST10)

Shakura, N.I., \& Sunyaev, R.A. 1973, A\&A, 24, 337 (SS73)

Shaposhnikov, N., \& Titarchuk, L. 2009, ApJ, 699, 453(ST09)

Shaposhnikov, N., \& Titarchuk, L. 2006, ApJ, 643, 1098 
Shrader, C.R., Titarchuk, L. \& Shaposhnikov, N., 2010, ApJ, 718, 488

Steiner, J. F., Reis, R. C., McClintock, J. E., et al. 2011, MNRAS, 416, 941

Stiele, H., Belloni, T. M., Kalemci, E., \& Motta, S. 2013, MNRAS, 429, 2655

Sunyaev, R.A. \& Titarchuk, L.G. 1980, A\&A, 86, 121 (ST80)

Titarchuk, L. 1994, ApJ, 434, 570

Titarchuk, L.G. \& Fiorito, R. 2004, ApJ, 612, 988 (TF04)

Titarchuk, L.G., Lapidus, I. \& Muslimov, A. 1998, ApJ, 499, 315

Titarchuk, L. \& Laurent, P. 2002, The Ninth Marcel Grossmann Meeting, Eds. by V. G. Gurzadyan , R. T. Jantzen, \& R. Ruffini; World Scientific Publishing Co.

Titarchuk, L. \& Laurent, P. 2002, meeting abstract of 4th COSPAR Scientific Assembly, The Second World Space Congress, held on October 10-19, 2002 in Houston, TX, USA

Titarchuk, L. \& Seifina, E. 2019 submitted to ApJ.

Titarchuk, L. \& Seifina, E. 2017, A\&A, 602, 113 (TS17)

Titarchuk, L. \& Seifina, E. 2009, ApJ, 706, 1463 (TS09)

Titarchuk, L., Shaposhnikov, N. \& Arefiev, V. 2007, ApJ, 660, 556 (TSA07)

Titarchuk, L. \& Shaposhnikov, N. 2005, ApJ, 626, 298

Titarchuk, L.G., Mastichiadis, A. \& Kylafis, N.D. 1997, ApJ, 487, 834

Titarchuk, L.G. \& Zannias, T. 1998, ApJ, 493, 863

Tomsick, J. A. et al. 2018, ApJ, 855, 3

Tomsick, J. A. et al. 2005, ApJ, 780, 78

Tomsick, J. A., Corbel, S., Goldwurm, A., \& Kaaret, Ph. 2005, ApJ, 630, 413

Trudolyubov, S., Churazov E. \& Gilfanov M. 1999, Astronomy Letters, 25, 718

Trudolyubov, S. 2001, ApJ, 558, 276

Vaughan, S. et al. 2016, MNRAS, 461, 3145

Vignarca, F., Migliari, S., Belloni, T., Psaltis, D., \& van der Klis, M. 2003, A\&A, 397, 729 
Walton, D.J. et al. 2016, ApJ, 826, 87

Wilms, J, Nowak, M. A., Pottschmidt, K., Pooley, G. G. \& Fritz, S. 2006, A\&A, 447, 245

$\dot{Z}$ ycki P. T., Done C., Smith D. A. 2001, MNRAS, 326, 1367

Życki, P. T., Krolik, J. H., Zdziarski, A. A., \& Kallman, T. R. 1994, ApJ, 437, 597

Życki, P. T., Done, C., \& Smith, D. A. 1999, MNRAS, 309, 561

Zdziarski, A. A. et al. 1996, MNRAS 283, 193 


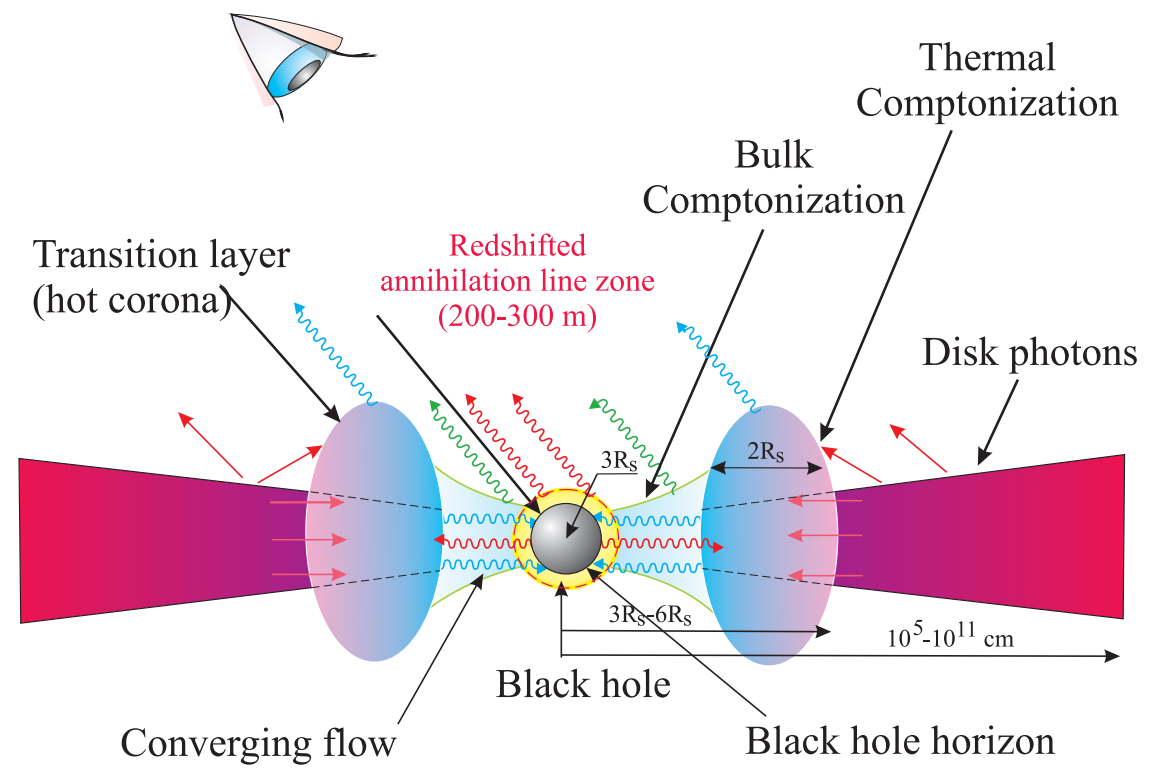

Fig. 1. - A proposed geometry for the thermal and bulk Comptonization regions in the binary hosting a BH. The thermal plus bulk Comptonization spectrum (green and blue arrrows) arises in the Compton cloud, where the disk BB seed photons undergo the thermal and dynamical Comptonization by infalling material. In addition, we take into account the region near a $\mathrm{BH}$ horizon, where the photon-photon interactions lead to the pair production effect. In this process the created positrons interact with accreting electrons there and therefore the annihilation line photons are created and distributed over the relatively narrow shell (enlarged in this plot) near the BH horizon. An Earth observer should see this annihilation line only at the gravitationally redshifted energies of the redshift of $z \gg 1$. 


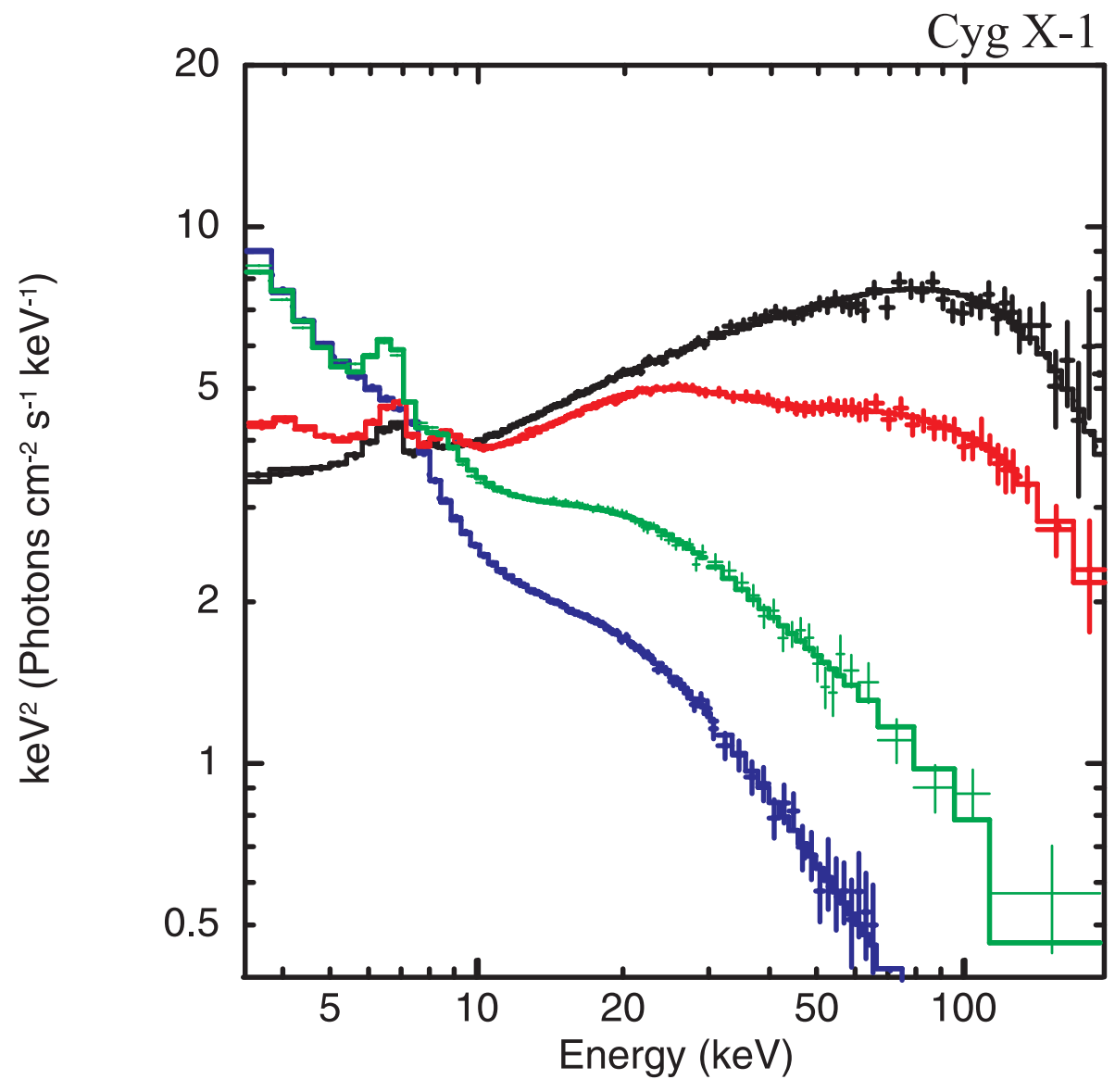

Fig. 2.- Four representative $E F_{E}$ spectral diagrams during LHS, IS, HSS and VHS spectral states of Cyg X-1. Data are taken from RXTE observations 40417-01-03-00 (black, LHS), 90127-01-01-08 (red, IS), 60090-01-02-00 (green, HSS), and 70414-01-01-02 (blue, VHS). 

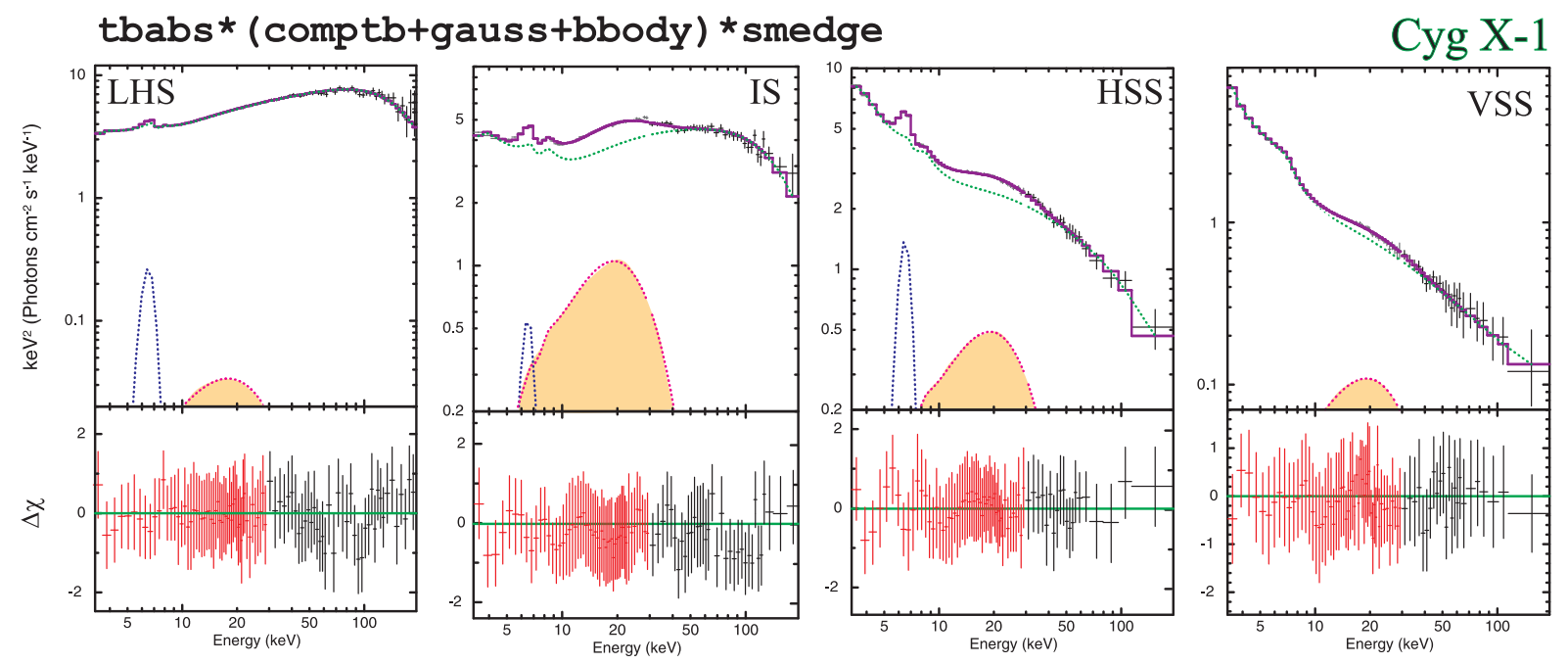

Fig. 3.- Evolution of to Cyg X-1 spectra using the tbabs* (comptb+gauss+bbody)*smedge model for four spectral states. Data are taken the RXTE observations: 40417-01-03-00 (LHS, left), 90127-01-01-08 (IS, left center), 60090-01-02-00 (HSS, right center), and 70414-01-0102 (VSS, right). The data are shown by black crosses and the spectral model components are displayed by dashed green, blue and pink lines for the comptb, gaussian, and Bbody, respectively. Yellow shaded areas demonstrate an evolution of the HBB component during evolution between the LHS, IS, HSS, and VSS states. 


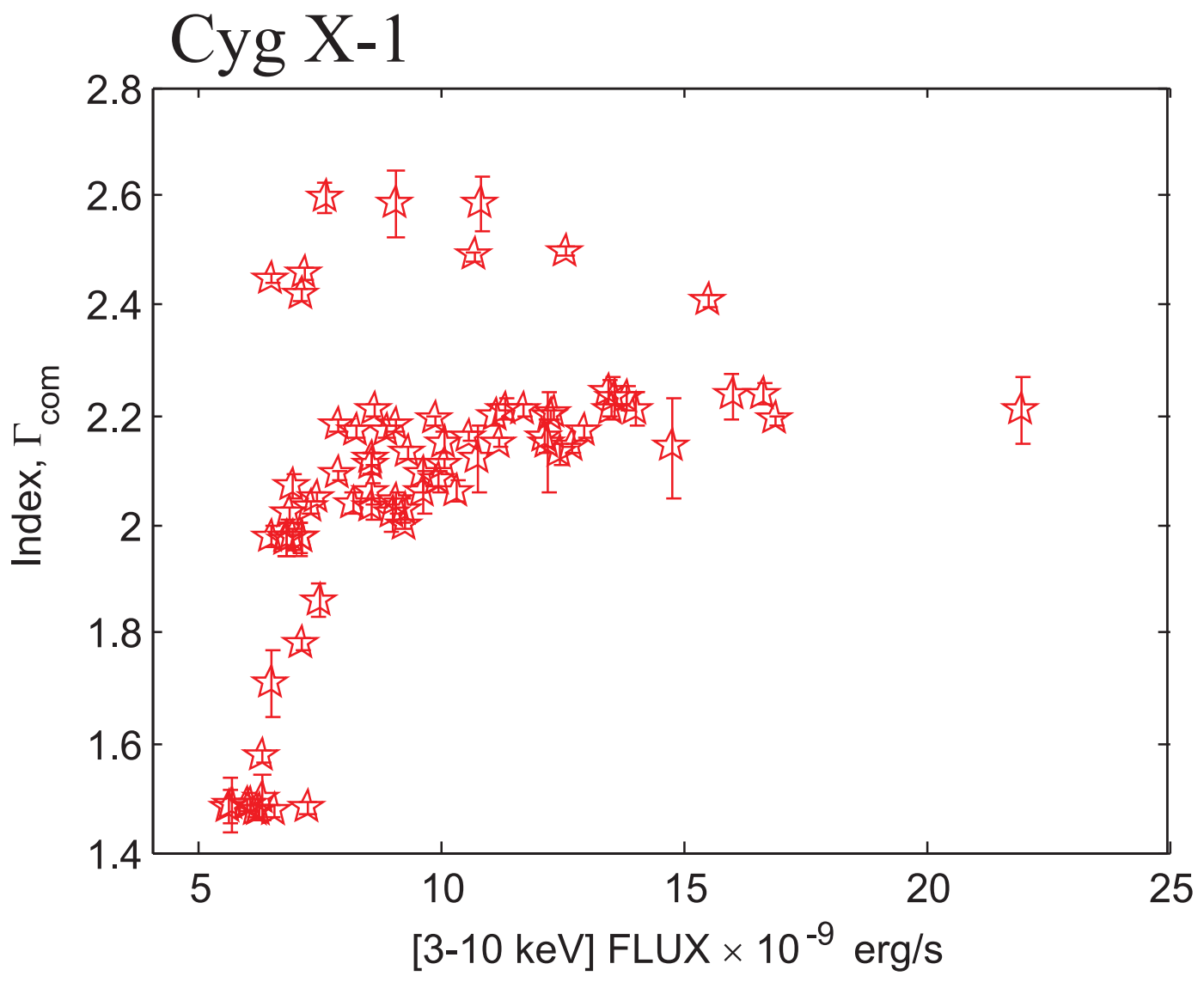

Fig. 4.- The photon index versus the $R X T E$ soft X-ray flux (3-10 keV) for Cyg X-1 using the Comptb model (left panel) and the reflection model (right panel). 

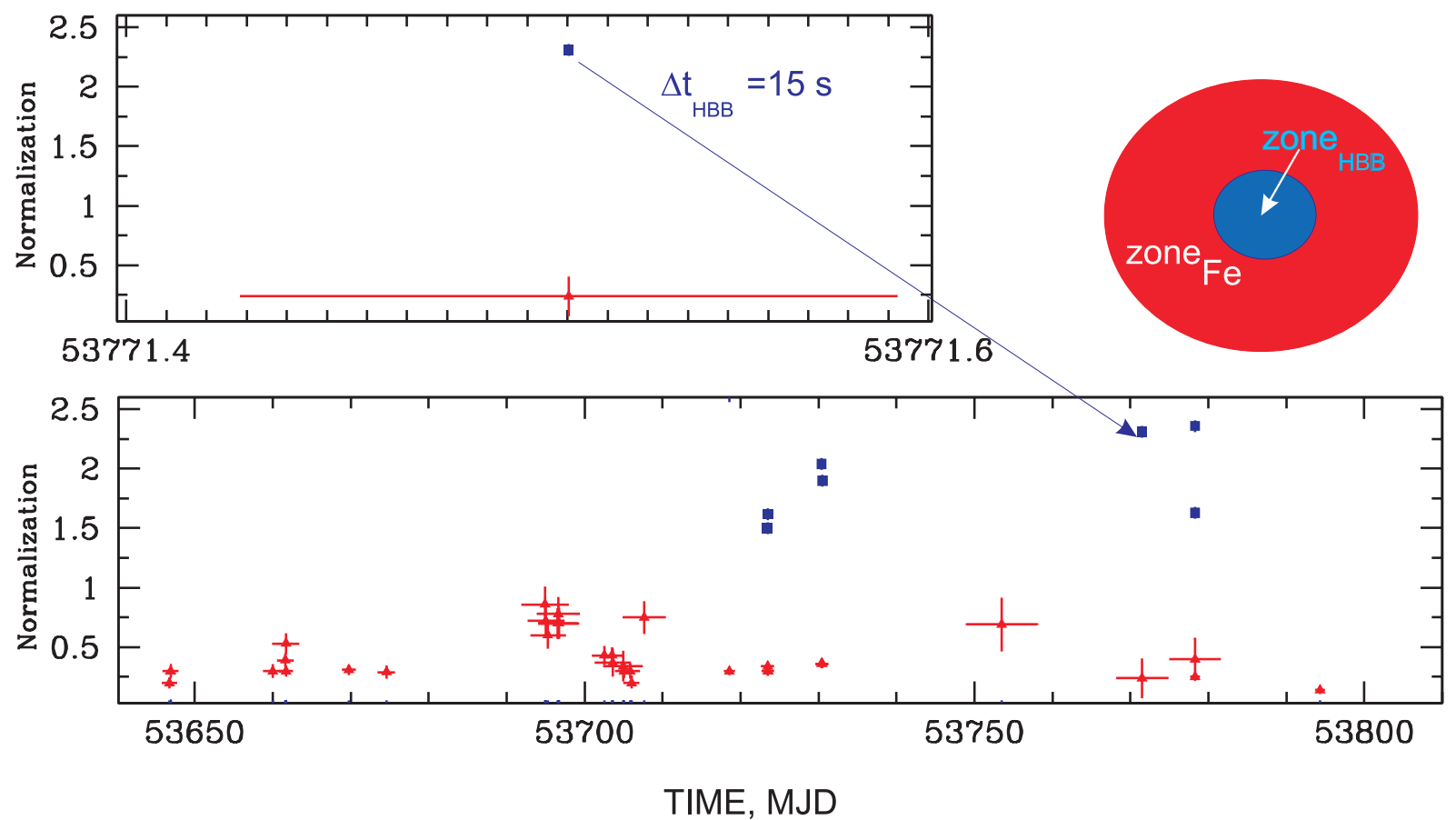

Fig. 5. - Time scales for spectral features: the HBB component (red) and Fe emission line modified by absorption edge (blue), as a function of the feature normalization in $L_{39} / d_{10}^{2}$ (for the $\mathrm{HBB}$ ) and total photons in units of $\mathrm{cm}^{-2} \mathrm{~s}^{-1}$ in the line for the Gaussian component (see Tables 6-9). 


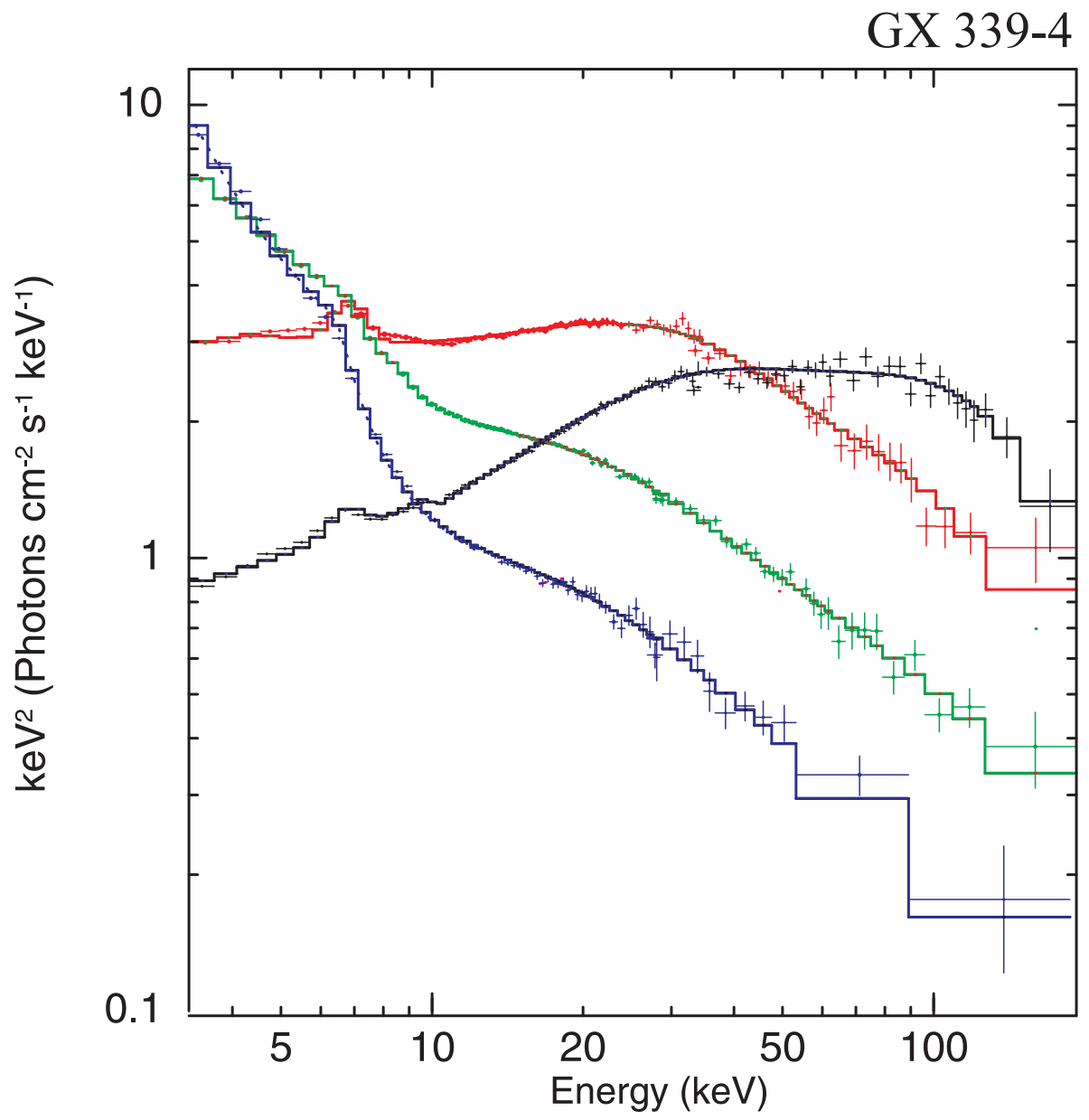

Fig. 6. - Four representative $E F_{E}$ spectral diagrams during the LHS, IS, HSS and VSS spectral states of GX 339-4. Data are taken from RXTE observations 92428-01-01-00 (blue, LHS), 92035-01-03-00 (green, IS), 60705-01-69-01 (pink, IS), 70109-01-07-00 (red, HSS), and 70109-01-07-00 (black, VSS). 

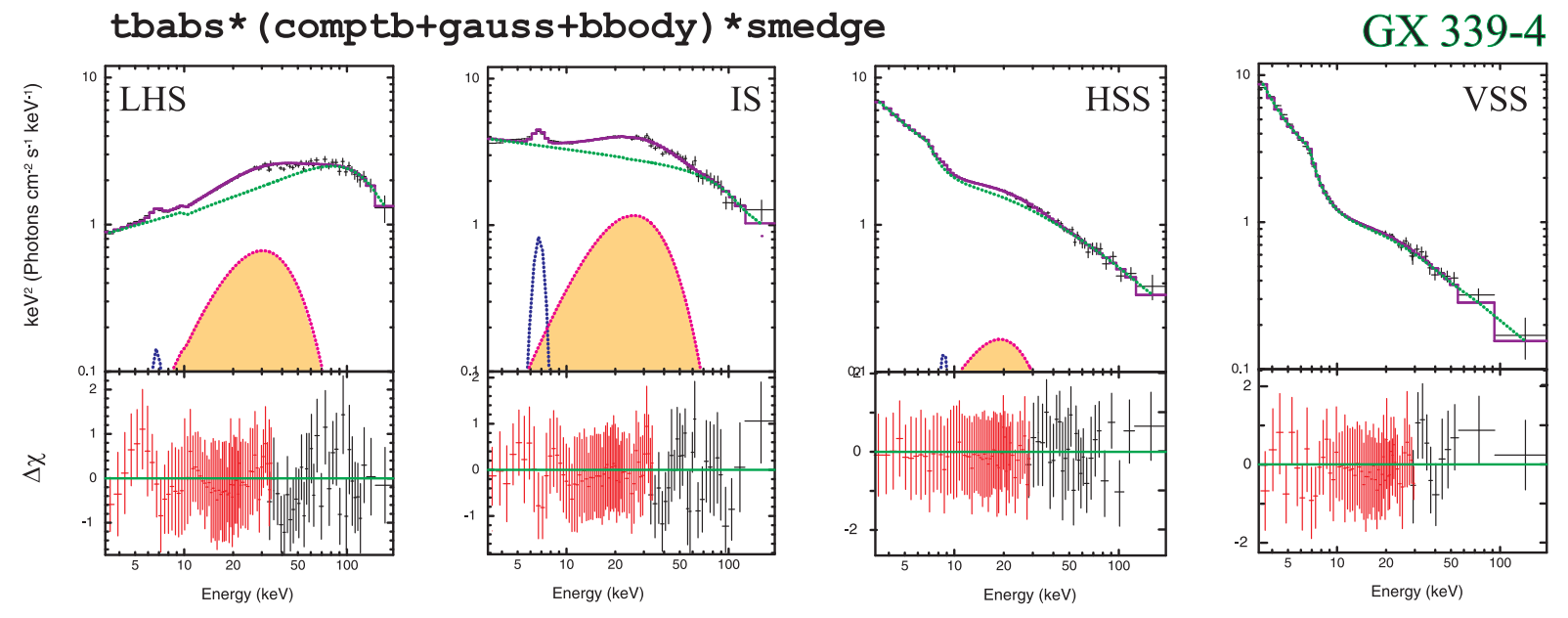

Fig. 7.- Evolution of to GX 339-4 spectra using the tbabs* (comptb+gauss+bbody)*smedge model for four spectral states. Data are taken the RXTE observations 92428-01-01-00 (LHS-IS, left), 92035-01-03-00 (IS, left center), 70109-04-01-01 (HSS, right center), and 70109-01-07-00 (VSS, right). The colors and model component designations are the same as those in Fig. 3.
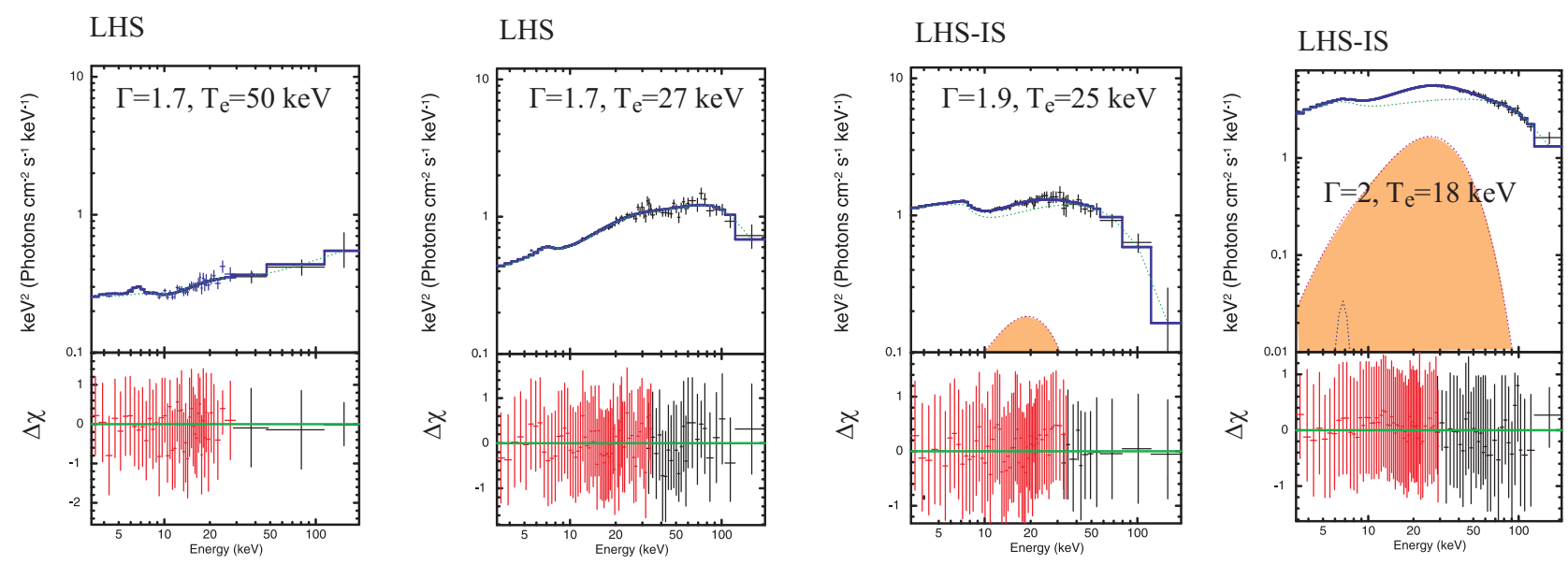

Fig. 8.- Evolution of GX 339-4 spectra between the LHS and IS states. Data are taken from the RXTE observations 70110-01-97-00 ( $k T_{e}=50 \mathrm{keV}, \Gamma=1.7$, LHS, left), 90418-01-01-01 $\left(k T_{e}=27 \mathrm{keV}, \Gamma=1.7\right.$, LHS, left center $), 60705-01-69-01\left(k T_{e}=25 \mathrm{keV}\right.$, $\Gamma=1.9$, LHS $\rightarrow$ IS, right center $)$, and 70110-01-08-00 $\left(k T_{e}=18 \mathrm{keV}, \Gamma=2.0\right.$, LHS $\rightarrow$ IS, right). The colors and model component designations are the same as in Fig. 3. Yellow shaded areas indicate the bbody component, of which presence/absence can be associated with the the decreese/increase of the electron temperature $k T_{e}$ in the framework of tbabs* (comptb+gauss+bbody)*smedge model. 


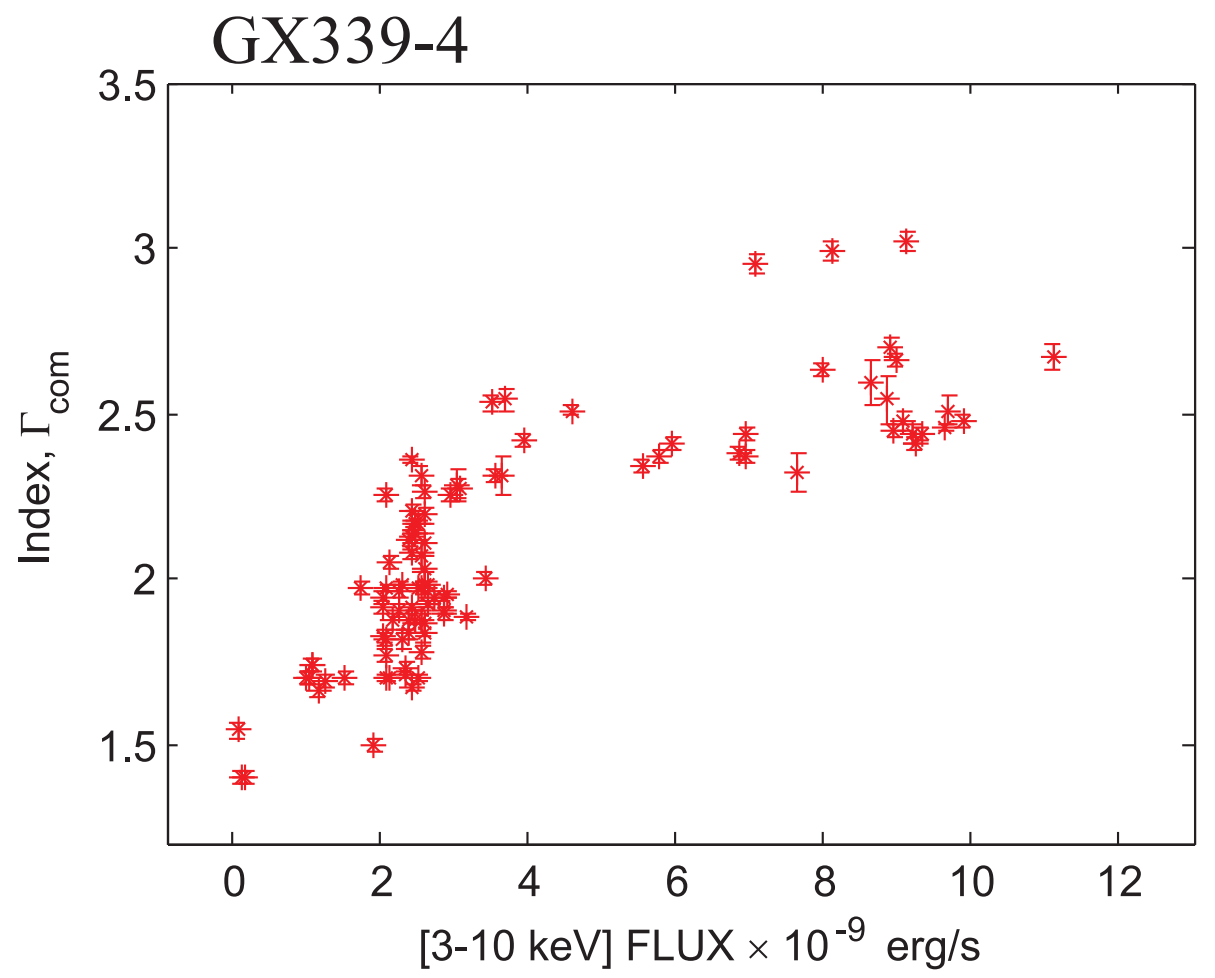

Fig. 9.- The photon index versus the RXTE soft X-ray flux (3-10 keV) for GX 339-4 using the Comptb model (left panel) and the reflection model (right panel). 


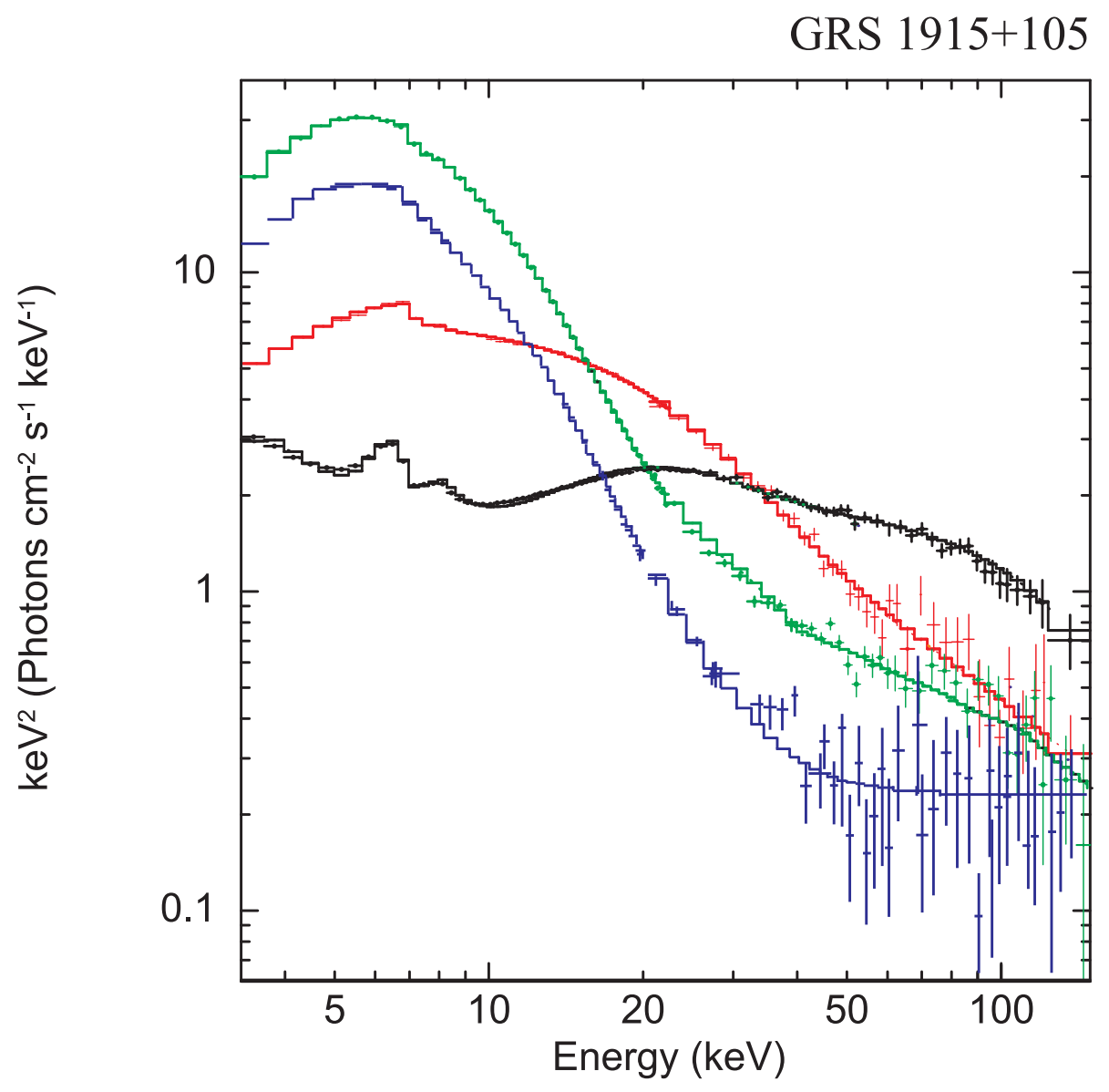

Fig. 10.- Four representative $E F_{E}$ spectral diagrams for the LHS (black), IS (red),HSS (green) and VSS (blue) for GRS 1915+105. Data are taken using RXTE observations. 

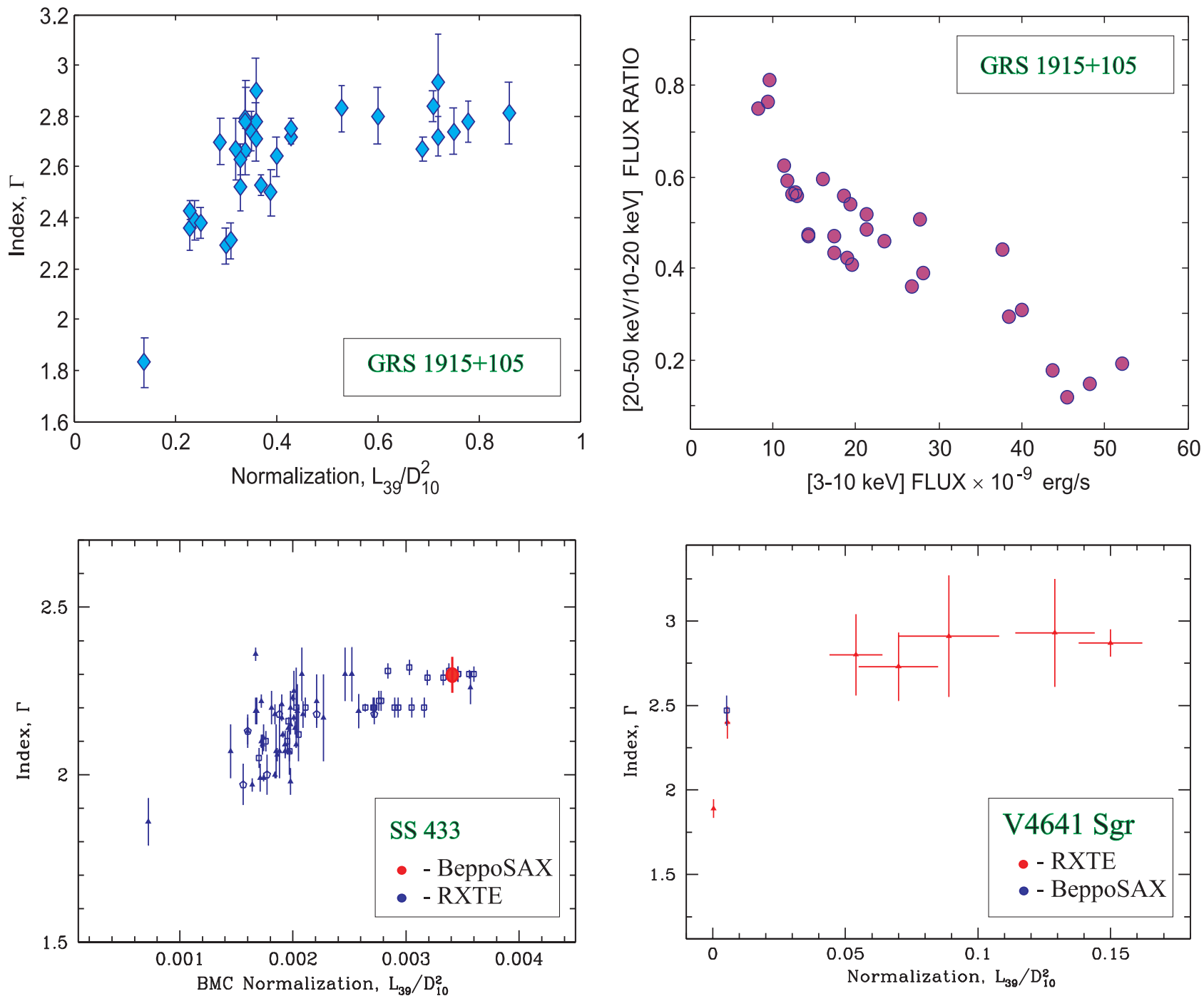

Fig. 11.- Top: The photon index, $\Gamma$ plotted vs. Comptb normalization, propotional to the disk mass accretion rate (left) and hardness-flux diagram (right) for the 2005 - 2006 transition of GRS 1915+105 analyzed using the tbabs*(comptb+gauss+bbody)*smedge model. Bottom: The photon index, $\Gamma$ plotted vs. Comptb normalization for SS 433 (left) and for V4641 Sgr (right), respectively. 

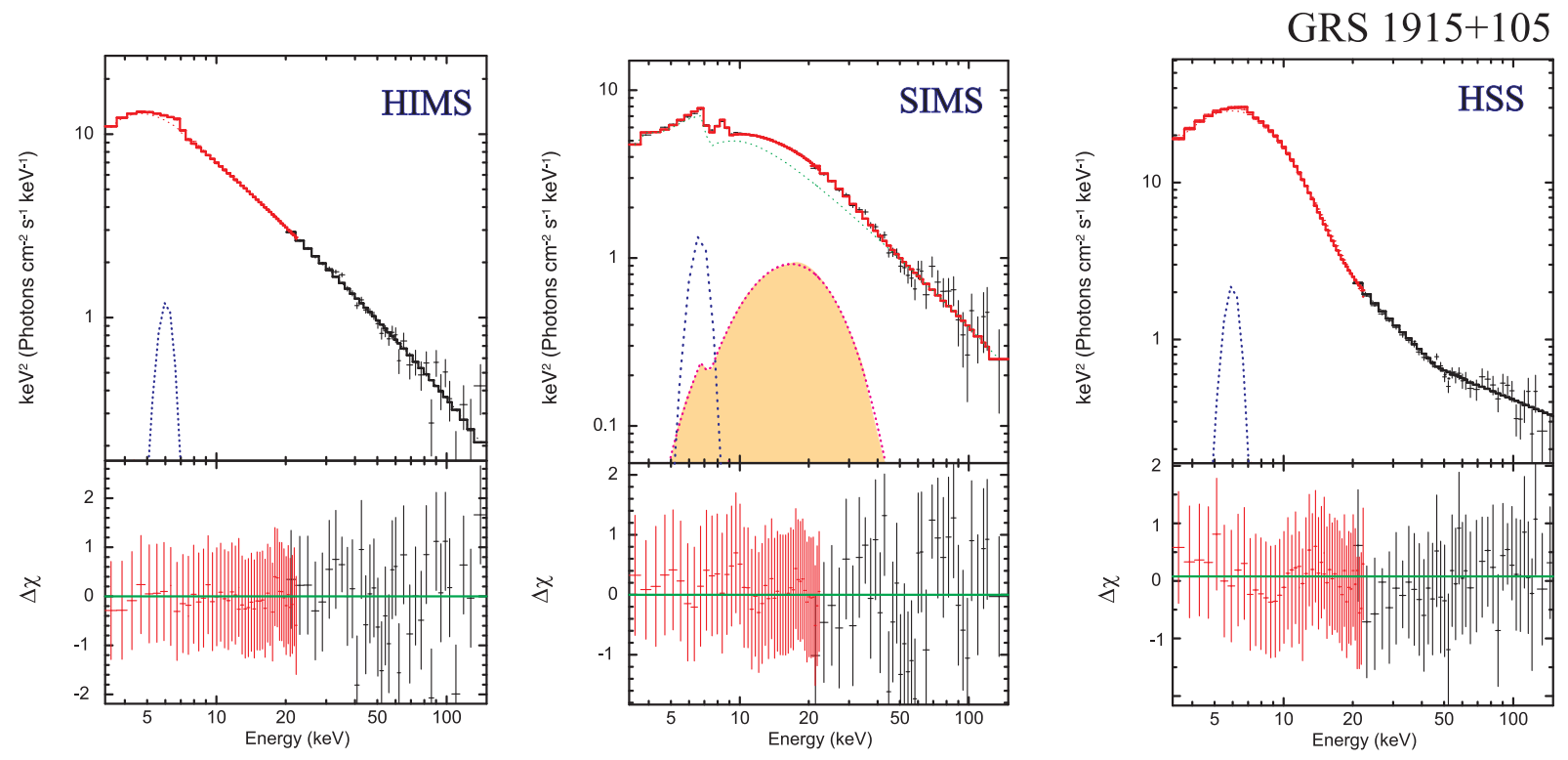

Fig. 12.- Examples of the best-fit spectra of GRS 1915+105 for three spectral states using tbabs* (comptb+gauss+bbody)*smedge model. Data are taken from RXTE observations 90024-02-43-00 (left, HIMS), 91701-01-49-00 (center, SIMS) and 92092-02-01-00 (right, HSS). 


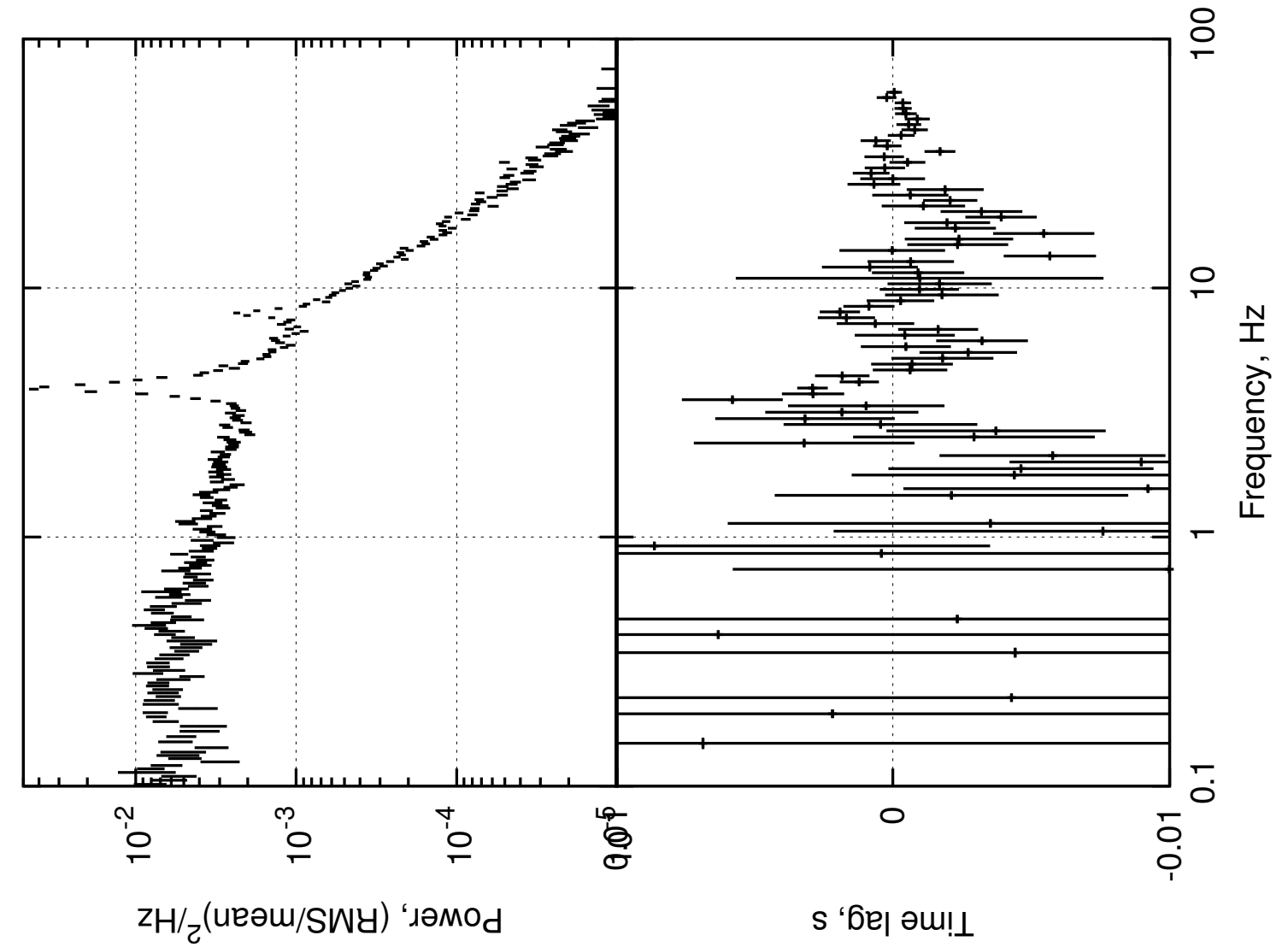

Fig. 13.- From top to bottom: evolutions of the flux density $S_{15 \mathrm{GHz}}$ at $15 \mathrm{GHz}$ (Ryle Telescope), the RXTE/ASM count rate, Comptb normalization, and the photon index, $\Gamma$ in the 2005 - 2006 transitions of GRS 1915+105 (MJD 53690 - 53855). All vertical strips mark the time intervals, in which the HBB component was detected. A behavior of spectral parameters for the short time 10 - $30 \mathrm{~s}$ intervals (see Tables 14-15) occurred during an interval of $\mathrm{MJD}=53771.51-53772.1$ (pink vertical strip, 91701-01-49-00 observation). 
SS 433

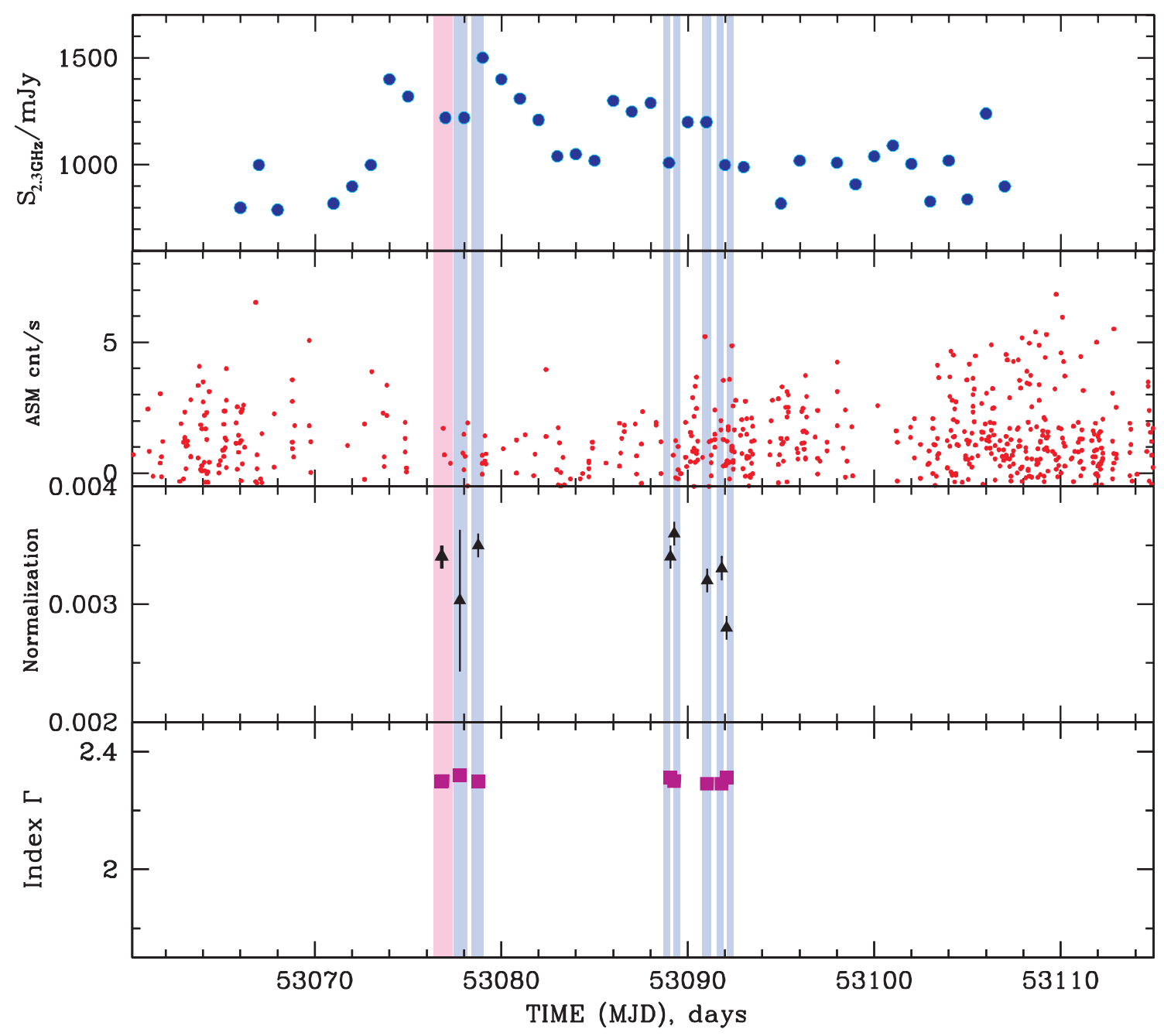

Fig. 14.- From top to bottom: evolutions of the flux density, $S_{2.3 G H z}$ at $2.3 \mathrm{GHz}$ (RATAN600 ), RXTE/ASM count rate, Comptb normalization, and the photon index, $\Gamma$ during March - April 2004 transition of SS 433 (MJD 53060 - 53114). Vertical strips mark the time intervals, in which an additional spectral component, HBB was detected. Pink vertical strip indicates the time interval of a simultaneous INTEGRAL and RXTE observation of SS 433. 

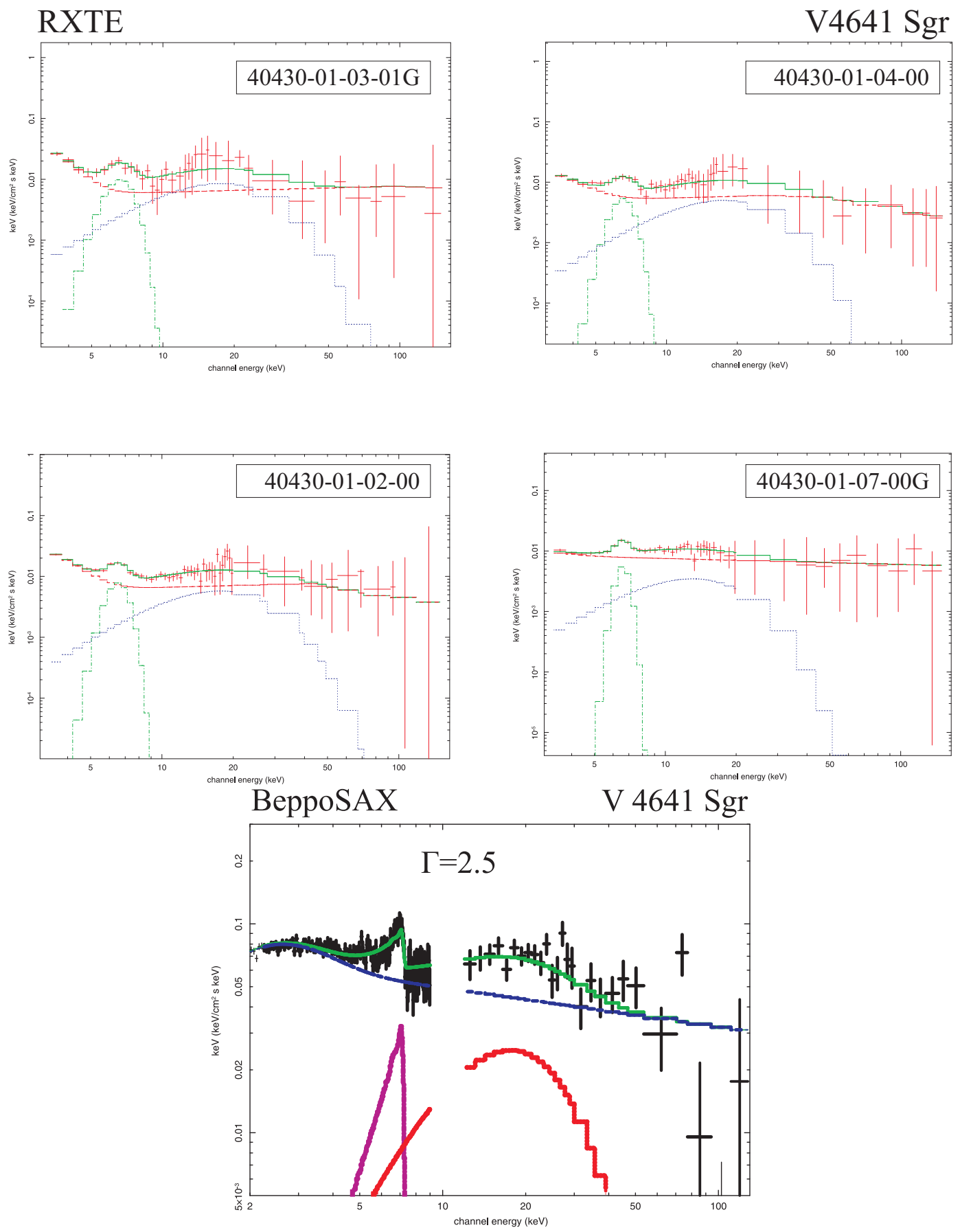

Fig. 15. - We apply a model, tbabs*(comptb+gauss+bbody)*smedge to the BH binary V4641 spectra (see Tables 17-18) and detected the HBB in four RXTE spectra of V4641 Sgr: 40430-01-02-00, 40430-01-03-01G, 40430-01-04-00, 40430-01-07-00G and in the BeppoSAX spectrum. Top: the data are shown by red crosses and the spectral model components are displayed by dashed red, green and blue lines for the Comptb, gaussian, and HBB, respectively. Bottom: the data are shown by black crosses and the spectral model components are displayed by blue, pink and red lines for the Comptb, Laor, and HBB, respectively. 


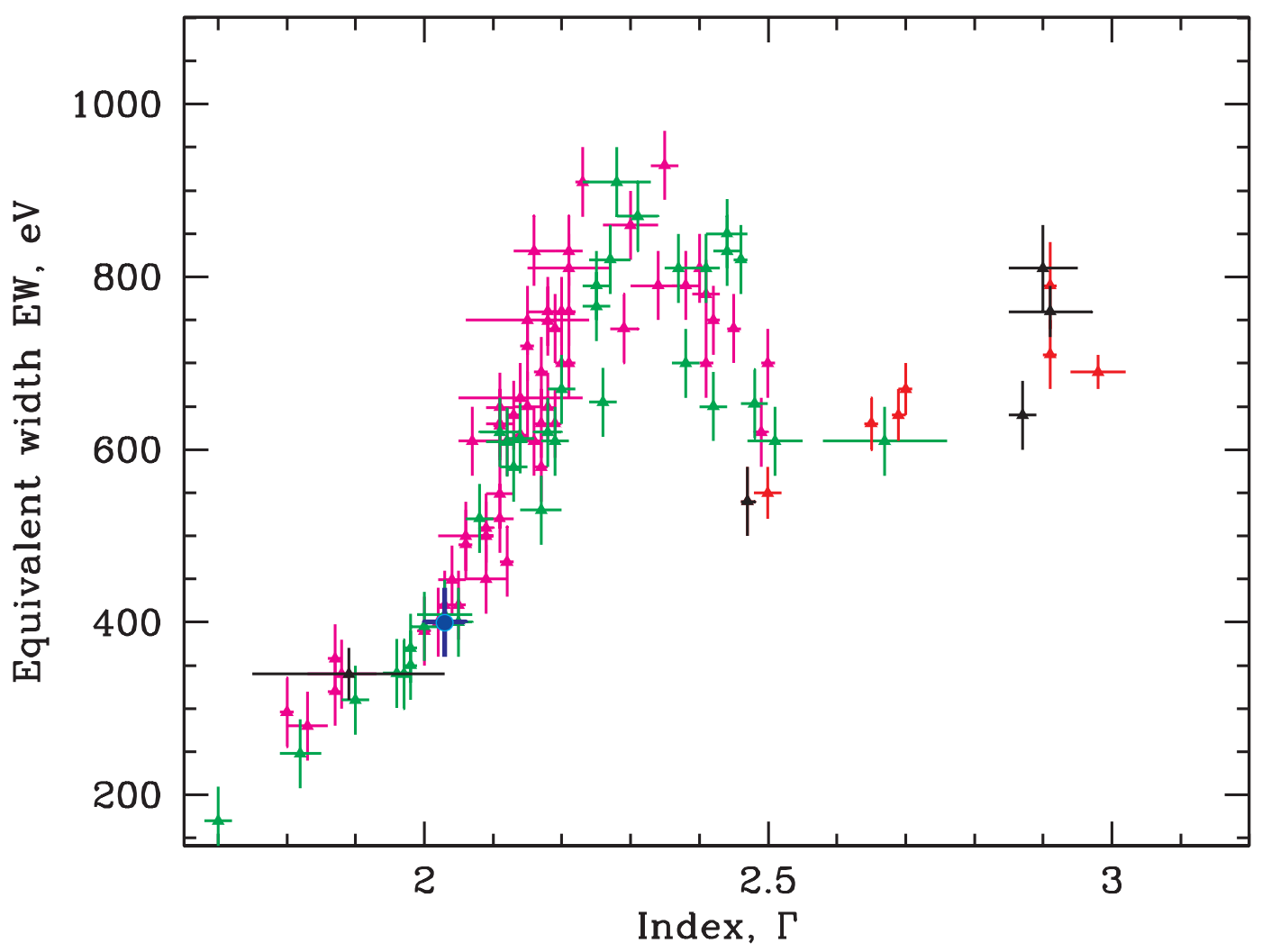

Fig. 16. - Equivalent width of the $\mathrm{HBB}$ in $\mathrm{eV}$ as a function of the photon index, $\Gamma$ for different sources: Cyg X-1 (pink), GX 339-4 (green), SS 433 (blue), GRS 1915+105 (red) and V4641 Sgr (black). 
Table 1. Summary of $R X T E$ Archive Data on Cyg X-1.

\begin{tabular}{|c|c|c|c|c|c|}
\hline Proposal ID & Start Date & Stop Date & $\begin{array}{c}\text { Type of } \\
\text { spectral state }\end{array}$ & $\begin{array}{c}\text { Index, } \\
\Gamma=\alpha+1^{\dagger}\end{array}$ & $\begin{array}{c}\text { Number of } \\
\text { HBB events }\end{array}$ \\
\hline 10235-01 ............. & $1996 \mathrm{Feb} 12$ & 1996 Feb 17 & LHS & 1.5 & $\ldots \ldots$. \\
\hline 10236-01 ............ & 1996 Dec 15 & 1996 Dec 18 & LHS & 1.7 & $\ldots \ldots$. \\
\hline 10238-01 ............. & 1996 Mar 26 & 1997 Feb 03 & LHS & 1.7 & $\ldots \ldots$. \\
\hline 10240-01 ........... & 1996 Feb 12 & 1996 Feb 19 & LHS & 1.7 & $\ldots \ldots$. \\
\hline 10241-01 ............ & 1996 Oct 23 & 1996 Oct 24 & LHS & 1.8 & $\ldots \ldots$. \\
\hline 10412-01 ............. & 1996 May 22 & 1996 Aug 12 & IS & $2.1-2.2$ & 4 \\
\hline 10512-01 ........... & 1996 Jun 04 & 1996 Jun 1 & IS & 2.3 & 3 \\
\hline 20173-01 ............ & 1997 Jan 17 & 1997 Jan 20 & LHS & 1.7 & $\ldots \ldots$ \\
\hline 20175-01 ............ & 1997 Jun 25 & 1998 Jan 02 & LHS & $1.7-1.9$ & $\ldots \ldots$. \\
\hline 30155-01 ............ & 1998 Dec 12 & 199 Dec 28 & LHS & 1.7 & $\ldots \ldots$. \\
\hline 30157-01 ............ & 1997 Dec 11 & 1998 Dec 09 & LHS & $1.7-1.8$ & $\ldots \ldots$. \\
\hline 30158-01 ........... & 1997 Dec 10 & 1997 Dec 30 & LHS & 1.7 & …... \\
\hline 30162-01 ............ & $1998 \mathrm{Feb} 12$ & 1996 Feb 17 & LHS & 1.7 & $\ldots \ldots$. \\
\hline 40099-01 ............ & 1999 Jan 14 & 2000 Feb 11 & LHS & 1.7 & $\ldots \ldots$. \\
\hline 40100-01 ............. & 1998 Dec 25 & 2002 Feb 14 & LHS & $1.7-1.8$ & $\ldots \ldots$. \\
\hline 40417-01 ............... & 1999 Apr 25 & 1999 Jun 13 & LHS & 1.7 & $\ldots \ldots$. \\
\hline 40101-01 ............ & 1999 Sep 27 & 1999 Oct 10 & IS & 2.2 & 7 \\
\hline 40102-01 ............ & 2000 Jan 05 & 2000 Jan 10 & LHS & 1.7 & $\ldots \ldots$. \\
\hline 50110-01 ........... & 2000 Feb 11 & 2002 Apr 06 & LHS-IS-HSS & $1.7-2.9$ & ....... \\
\hline 50109-03 …........ & 2000 Nov 05 & 2001 Feb 08 & LHS-IS & $1.8-2.2$ & 14 \\
\hline 50109-03 ............ & 2000 Nov 05 & 2001 Jan 11 & LHS-IS & $1.8-2.2$ & 11 \\
\hline 50119-01 ............. & 2000 Oct 28 & 2001 Jan 04 & LHS-IS & $1.8-2.2$ & 12 \\
\hline 50109-01 ........... & 2000 Dec 22 & 2001 Feb 15 & LHS-IS & $1.8-2.3$ & 11 \\
\hline 60089-01 ............ & 2001 Aug 19 & 2001 Aug 30 & LHS-IS & $1.8-2.2$ & ........ \\
\hline 60089-02 ........... & 2001 Sep 23 & 2001 Oct 28 & IS & $2.0-2.3$ & 6 \\
\hline 60089-03 ............ & 2001 Oct 07 & 2002 Feb 21 & IS & $2.2-2.4$ & 14 \\
\hline 60091-01 ............ & 2001 Oct 15 & 2001 Oct 22 & IS & $2.2-2.4$ & 4 \\
\hline 60090-01 ............ & 2002 Mar 08 & 2004 Apr 03 & LHS-IS & $1.8-2.7$ & 22 \\
\hline 70414-01 ............ & 2002 Jul 13 & 2002 Dec 29 & LHS & $1.7-1.9$ & $\ldots \ldots$. \\
\hline 70015-04 ........... & 2002 Sep 15 & 2002 Sep 17 & IS & $2.1-2.5$ & 9 \\
\hline 80110-01 ............ & 2004 Mar 09 & 2005 Mar 01 & LHS & $1.7-1.8$ & $\ldots \ldots$. \\
\hline 80111-01 ............. & 2003 Apr 19 & 2003 Apr 20 & LHS & 1.7 & -...... \\
\hline 90126-01 …......... & 2004 Apr 07 & 2004 Apr 11 & LHS & 1.7 & ........ \\
\hline 80113-01 ............ & 2004 May 17 & 2004 May 21 & LHS & 1.7 & ....... \\
\hline 90104-01 ............ & 2004 Nov 14 & 2004 Dec 12 & LHS & $1.7-1.8$ & ....... \\
\hline 90414-01 ............ & 2004 Dec 18 07:45:36 & 2004 Dec 18 08:25:36 & LHS & 1.7 & ........ \\
\hline $90127-01 \ldots \ldots \ldots \ldots$ & 2005 Feb 01 & 2005 Feb 08 & IS & $2.0-2.2$ & 33 \\
\hline
\end{tabular}


Table 1 - Continued

\begin{tabular}{|c|c|c|c|c|c|}
\hline Proposal ID & Start Date & Stop Date & $\begin{array}{c}\text { Type of } \\
\text { spectral state }\end{array}$ & $\begin{array}{c}\text { Index, } \\
\Gamma=\alpha+1^{\dagger}\end{array}$ & $\begin{array}{c}\text { Number of } \\
\text { HBB events }\end{array}$ \\
\hline 91096-01 …........ & 2005 Mar 06 & 2005 Jul 15 & LHS & $1.7-2.2$ & 9 \\
\hline
\end{tabular}

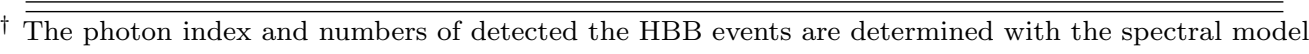
tbabs*(comptb+gauss+blackbody).

Table 2. Summary of RXTE Archive Data on GX 339-4.

\begin{tabular}{|c|c|c|c|c|c|}
\hline Proposal ID & Start Date & Stop Date & $\begin{array}{c}\text { Type of } \\
\text { spectral state }\end{array}$ & $\begin{array}{c}\text { Index, } \\
\Gamma=\alpha+1^{\dagger}\end{array}$ & $\begin{array}{c}\text { Number of } \\
\text { HBB events }\end{array}$ \\
\hline 40031-03 ........... & 2002 Apr 18 & 2002 May 20 & LHS & 1.9 & $\ldots \ldots$. \\
\hline 50117-01 ........... & 2001 Feb 25 & 2003 Mar 20 & IS & $2.1-2.3$ & $\ldots \ldots$ \\
\hline 60705-01 …........ & 2001 Mar 06 & 2006 Jun 10 & LHS-IS & $1.8-2.1$ & 2 \\
\hline 70108-03 ............ & 2002 May 06 & 2002 Jun 14 & IS & 2.0 & 1 \\
\hline 70109-01 ........... & 2002 Apr 07 & 2003 Feb 24 & LHS-IS-HSS & $1.9-3.0$ & $\ldots \ldots$ \\
\hline 70109-02 …........ & 2003 Mar 11 & 2003 Mar 15 & IS-IS & $2.1-2.3$ & 1 \\
\hline 70109-03 ............ & 2002 Jun 15 & 2002 Jun 16 & IS & $2.2-2.4$ & ....... \\
\hline 70109-04 …......... & 2002 May 05 & 2002 May 12 & HSS & $2.6-2.7$ & ....... \\
\hline 70110-01 …........ & 2002 Apr 15 & 2003 Feb 24 & IS-HSS & $1.6-2.6$ & 4 \\
\hline 70111-01 ............ & 2003 Feb 20 13:36:16 & 2003 Feb 20 19:36:00 & IS & 2.5 & $\ldots \ldots$. \\
\hline 70128-02 …........ & 2003 Feb 20 & 2003 Apr 13 & LHS-IS & $1.7-2.4$ & ........ \\
\hline 80102-01 ........... & 2003 May 04 & 2004 Jul 01 & LHS & 1.8 & $\ldots \ldots$ \\
\hline 80116-01 ............ & 2003 Apr 06 & 2003 Apr 13 & LHS & 1.7 & $\ldots \ldots$ \\
\hline 90110-02 …....... & 2004 Aug 15 & 2004 Aug 16 & IS & $2.2-2.4$ & 4 \\
\hline 90704-01 …........ & 2004 Aug 09 & 2005 May 12 & LHS-IS-HSS & $1.9-2.5$ & 2 \\
\hline 90704-02 …........ & 2004 Aug 20 07:45:20 & 2004 Aug 20 16:42:40 & IS-HSS & $2.3-3.0$ & $\cdots \cdots$ \\
\hline 90418-01 ............ & 2004 Jul 11 & 2004 Jul 13 & IS-HSS & $2.1-2.6$ & 8 \\
\hline 92035-01 ............ & 2007 Jan 29 & 2007 Feb 18 & LHS & $1.6-1.9$ & ....... \\
\hline 92428-01 …......... & 2007 Jan 09 & 2007 Feb 14 & LHS-IS & $1.7-2.4$ & 5 \\
\hline 92704-03 ........... & 2007 Apr 21 & 2007 Jul 18 & LHS-IS & $1.9-2.5$ & -...... \\
\hline 92704-04 …......... & 2007 May 15 & 2007 Jun 05 & LHS-IS & $2.3-3.0$ & ........ \\
\hline
\end{tabular}

$\dagger$ The photon index and numbers of detected HBB events are determined with the spectral model tbabs* (comptb+gauss+blackbody)*smedge. 
Table 3. The list of the GRS $1915+105$ observations by $R X T E$, where the HBB component was detected ${ }^{\dagger}$.

\begin{tabular}{|c|c|c|c|c|}
\hline Obs. ID & Start time (UT) & End time (UT) & MJD interval & Ref \\
\hline $91701-01-22-00$ & 2006 Feb 28 07:33:52 & 2006 Feb 28 08:01:36 & $53794.31-53794.33$ & 1,3 \\
\hline 91701-01-31-00 & 2005 Oct 3 18:24:32 & 2005 Oct 3 18:53:36 & $53646.76-53646.78$ & $1,2,3$ \\
\hline 91701-01-33-00 & 2005 Oct 18 16:57:36 & 2005 Oct 18 20:49:36 & $53661.70-53661.86$ & $1,2,3$ \\
\hline 91701-01-33-01 & 2005 Oct 16 23:35:12 & 2005 Oct 17 01:54:56 & $53659.98-53660.07$ & $1,2,3$ \\
\hline 91701-01-34-00 & 2005 Oct 26 16:36:00 & 2005 Oct 26 17:19:44 & $53669.69-53669.72$ & $1,2,3$ \\
\hline 91701-01-34-01 & 2005 Oct 26 18:10:24 & 2005 Oct 26 19:07:44 & $53669.75-53669.79$ & $1,2,3$ \\
\hline $1-01-35-00$ & 2005 Oct 31 14:15:28 & 2005 Oct 31 16:46:40 & $53674.59-53674.70$ & $1,2,3$ \\
\hline 91701-01-38-00 & 2005 Nov 22 13:32:33 & 2005 Nov 22 14:02:40 & $53696.56-53696.58$ & $1,2,3$ \\
\hline 91701-01-38-01 & 2005 Nov 22 15:10:10 & 2005 Nov 22 15:38:40 & $53696.63-53696.65$ & $1,2,3$ \\
\hline 91701-01-39-00 & 2005 Nov 29 13:44:16 & 2005 Nov 29 14:21:36 & $53703.57-53703.60$ & $1,2,3$ \\
\hline 91701-01-39-01 & 2005 Nov 29 15:20:32 & 2005 Nov 29 15:52:32 & $53703.63-53703.66$ & $1,2,3$ \\
\hline 91701-01-40-00 & 2005 Dec 7 13:14:40 & 2005 Dec 7 17:05:36 & $53702.52-53711.71$ & 1,3 \\
\hline 91701-01-41-00 & 2005 Dec 14 13:39:44 & 2005 Dec 14 15:57:36 & $53718.56-53718.66$ & $1,2,3$ \\
\hline $91701-01-42-00$ & 2005 Dec 19 11:21:04 & 2005 Dec 19 11:13:36 & $53723.47-53723.46$ & $1,2,3$ \\
\hline 91701-01-42-01 & 2005 Dec 19 13:01:52 & 2005 Dec 19 13:50:40 & $53723.54-53723.57$ & $1,2,3$ \\
\hline 91701-01-43-00 & 2005 Dec 26 10:00:48 & 2005 Dec 26 10:51:44 & $53730.41-53730.45$ & $1,2,3$ \\
\hline 91701-01-43-01 & 2005 Dec 26 12:17:04 & 2005 Dec 26 12:33:36 & $53730.51-53730.52$ & 1,3 \\
\hline 91701-01-46-00 & 2006 Jan 18 10:19:27 & 2006 Jan 18 10:57:35 & $53753.43-53753.45$ & $1,2,3$ \\
\hline 91701-01-49-00 & 2006 Feb 5 12:15:12 & 2006 Feb 5 13:03:44 & $53771.51-53771.54$ & $1,2,3$ \\
\hline 91701-01-50-00 & 2006 Feb 12 07:23:12 & 2006 Feb 12 08:20:32 & $53778.30-53778.34$ & $1,2,3$ \\
\hline 90024-02-37-00 & 2005 Oct 3 21:39:28 & 2005 Oct 3 22:08:32 & $53646.90-53646.92$ & 1,3 \\
\hline 90024-02-38-00 & 2005 Oct 18 15:04:00 & 2005 Oct 18 15:28:32 & $53661.62-53661.64$ & 1,3 \\
\hline 90024-02-40-00 & 2005 Nov 22 16:48:48 & 2005 Nov 22 17:07:44 & $53696.70-53696.71$ & 1,3 \\
\hline 90024-02-43-00 & 2006 Jan 18 12:13:35 & 2006 Jan 18 12:13:35 & $53753.51-53753.50$ & 1,3 \\
\hline 90105-06-03-00 & 2005 Nov 21 00:56:32 & 2005 Nov 21 05:02:40 & $53695.04-53695.21$ & 1,3 \\
\hline 90105-06-03-01 & 2005 Nov 20 21:47:44 & 2005 Nov 20 22:40:32 & $53694.91-53694.94$ & 1,3 \\
\hline 90105-06-03-02 & 2005 Nov 21 07:23:44 & 2005 Nov 21 07:56:32 & $53695.31-53695.33$ & 1,3 \\
\hline 92092-01-01-00 & 2005 Dec 1 01:30:56 & 2005 Dec 1 07:18:40 & $53705.06-53705.30$ & 1,3 \\
\hline 92092-01-01-01 & 2005 Nov 30 22:37:20 & 2005 Nov 30 23:20:32 & $53704.94-53704.97$ & 1,3 \\
\hline 92092-02-01-00 & 2005 Dec 1 20:22:56 & 2005 Dec 2 00:01:04 & $53705.84-53706.00$ & 1,3 \\
\hline 92092-02-01-01 & 2005 Dec 2 00:02:24 & 2005 Dec 2 01:15:44 & $53706.00-53706.05$ & 1,3 \\
\hline 92092-03-01-00 & 2005 Dec 3 16:25:04 & 2005 Dec 3 22:04:32 & $53707.68-53707.91$ & 1,3 \\
\hline
\end{tabular}

${ }^{\dagger}$ Total list of used observations is presented in TS09. References: (1) Rodriguez et al. (2008) (2) TS09, (3) this work.

Table 4. The V4641 Sgr observation using BeppoSAX and RXTE used in our analysis.

\begin{tabular}{llcccc}
\hline \hline Satellite & Obs. ID & Start time (UT) & End time (UT) & MJD interval & Ref \\
\hline BeppoSAX & 205490061 & 1999 March 13 17:49:16 & 1999 March 14 00:25:23 & $51250.7-51251.4$ & 1,2 \\
\hline
\end{tabular}




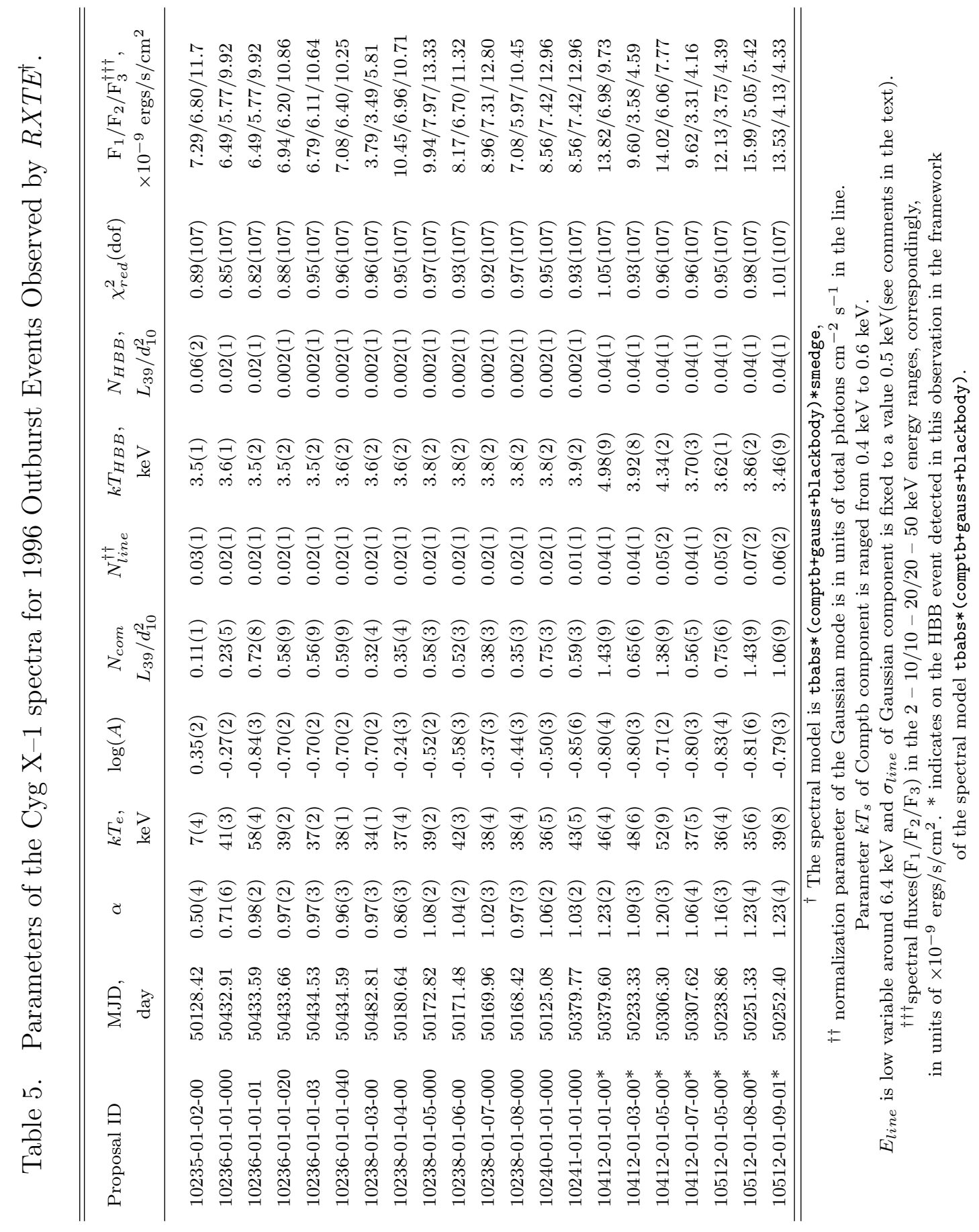




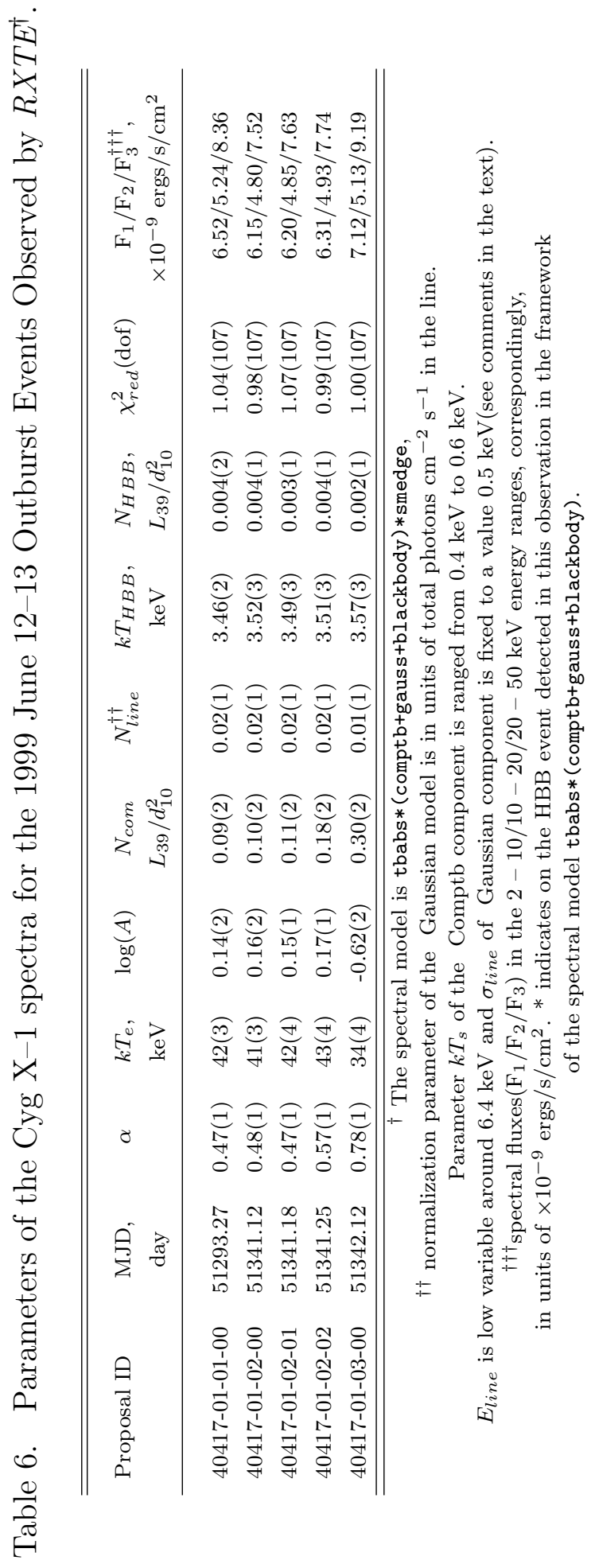




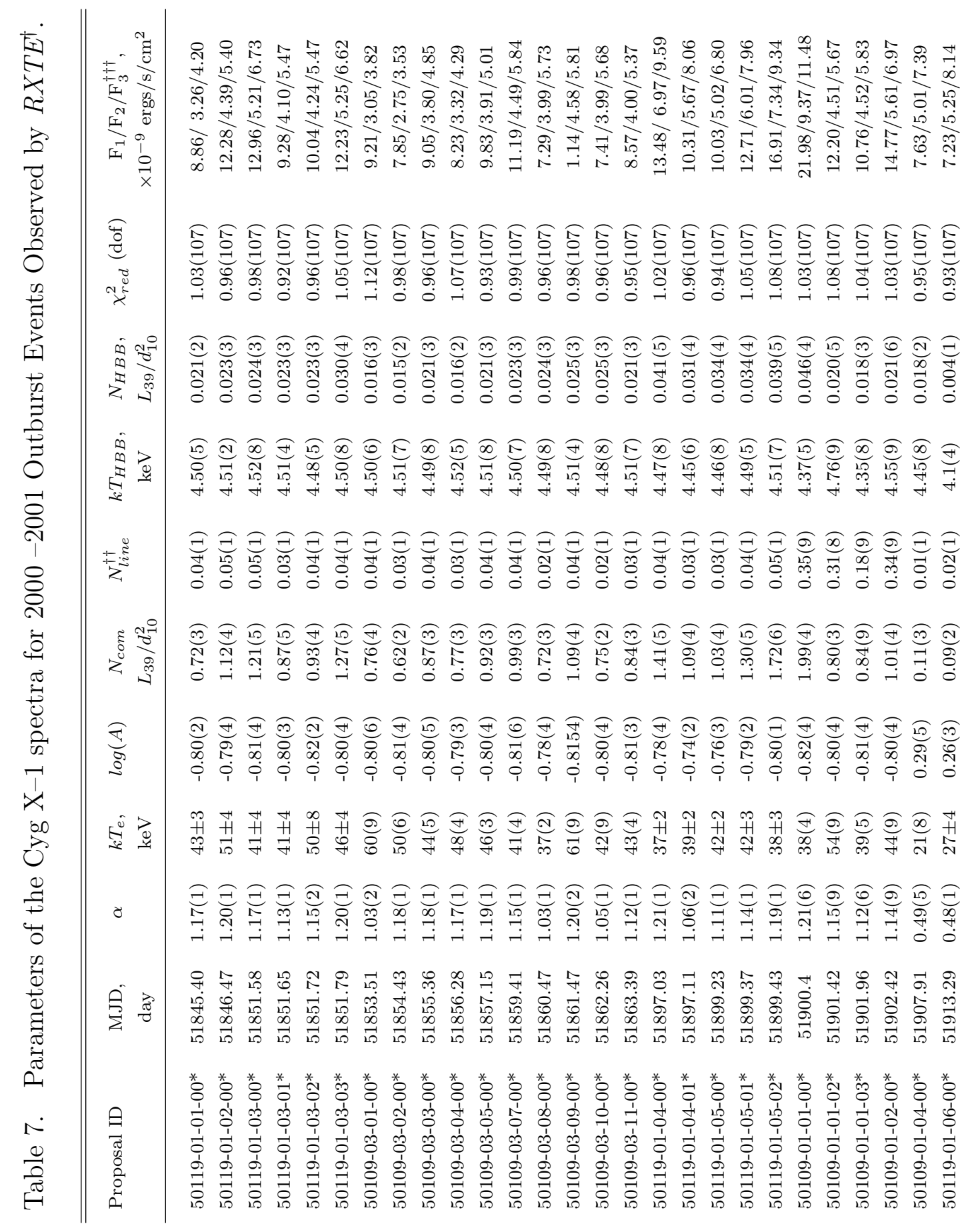




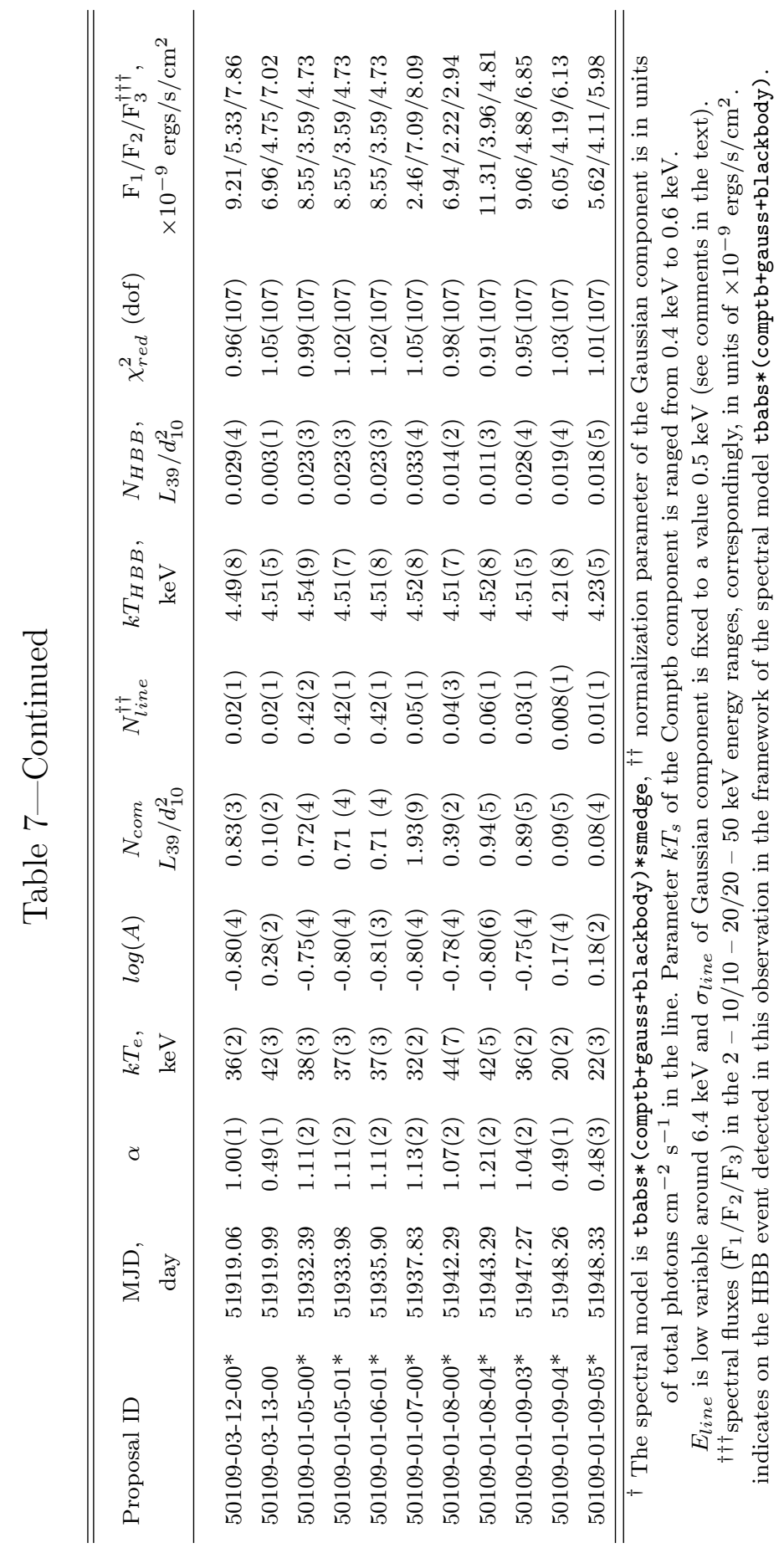




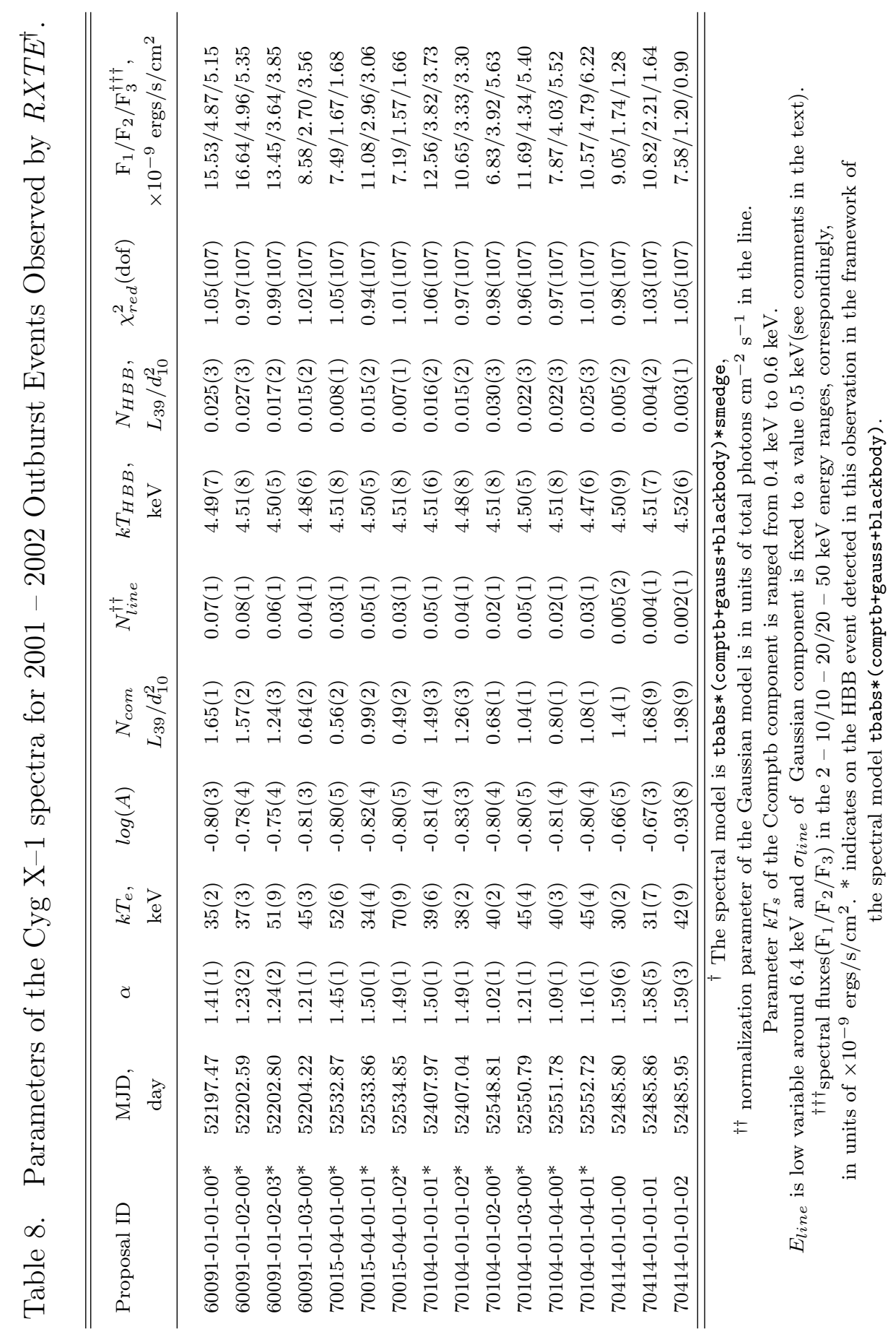




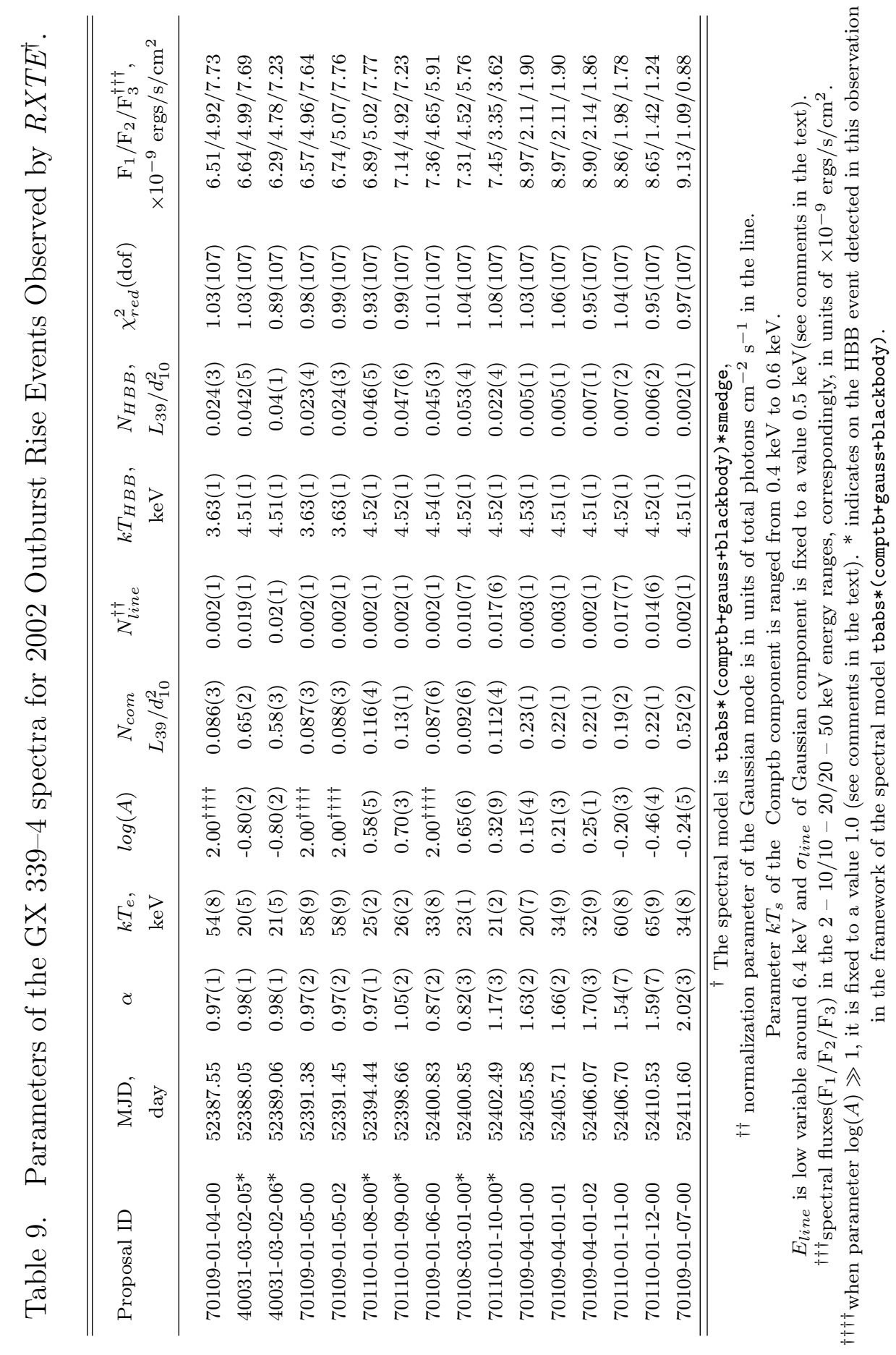




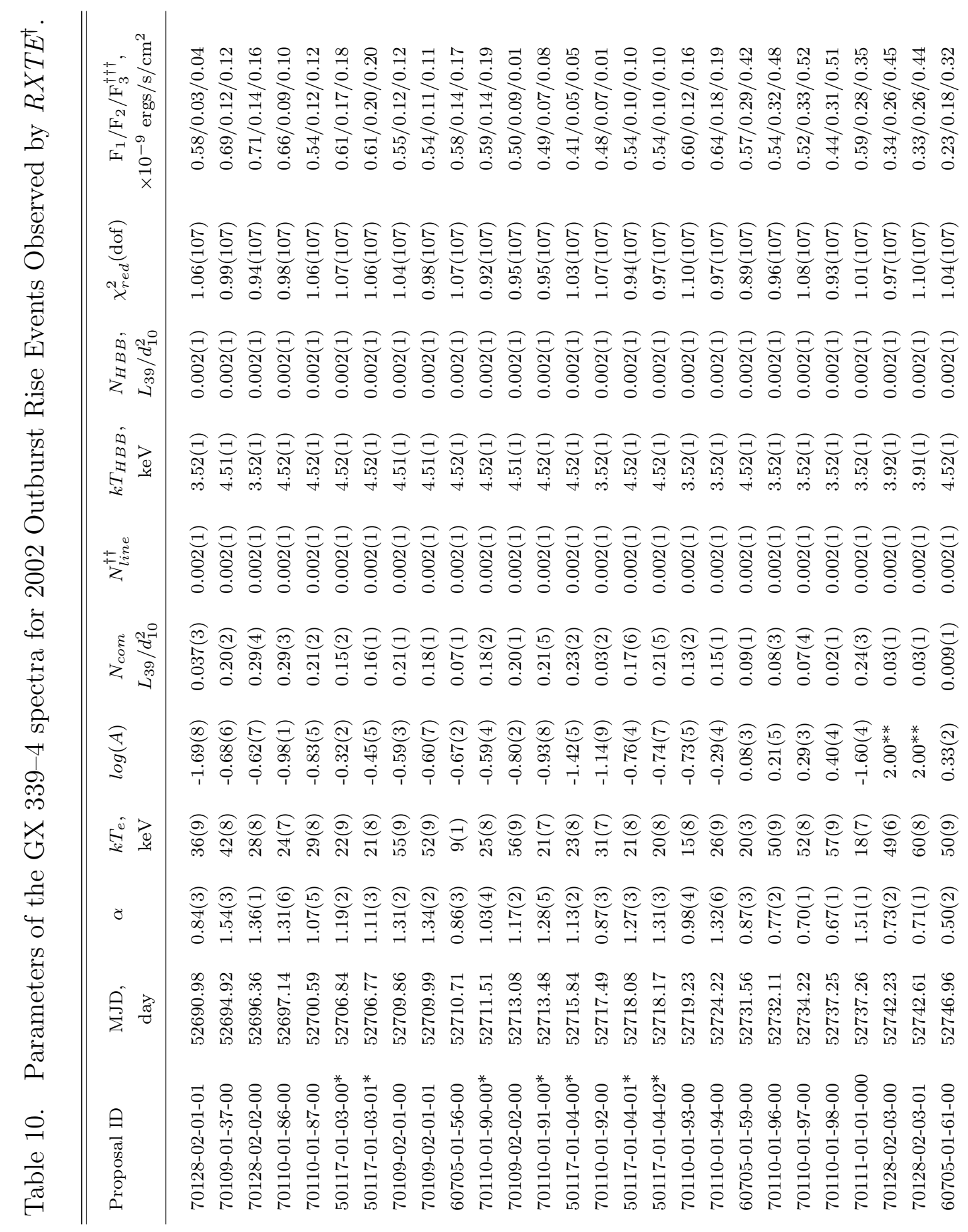




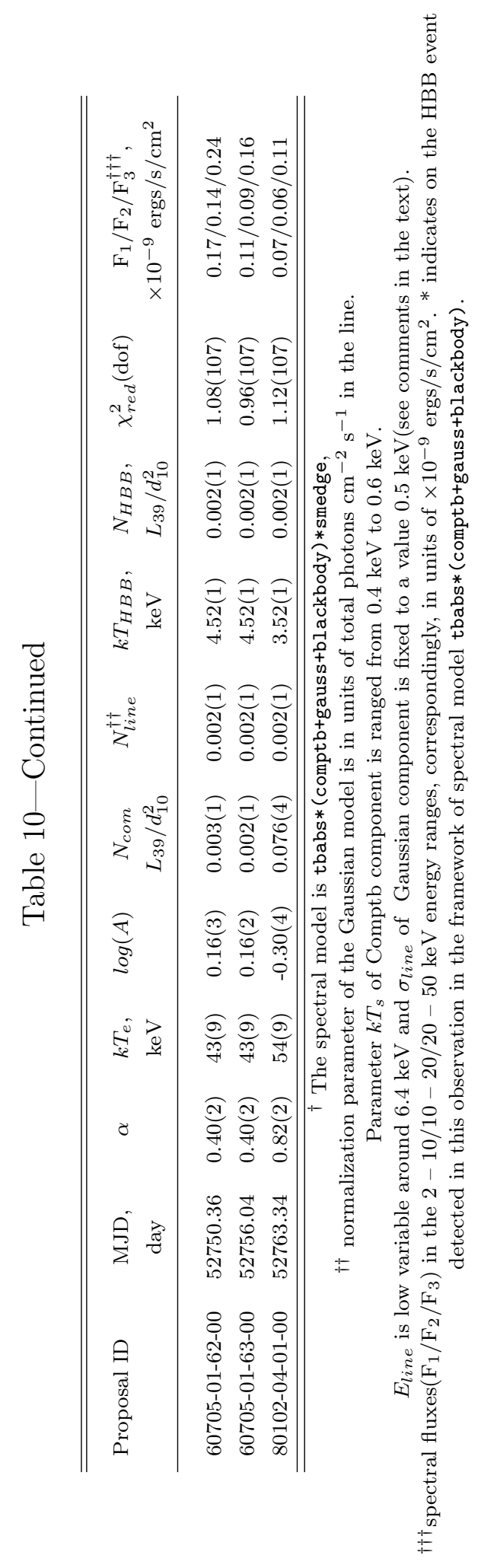




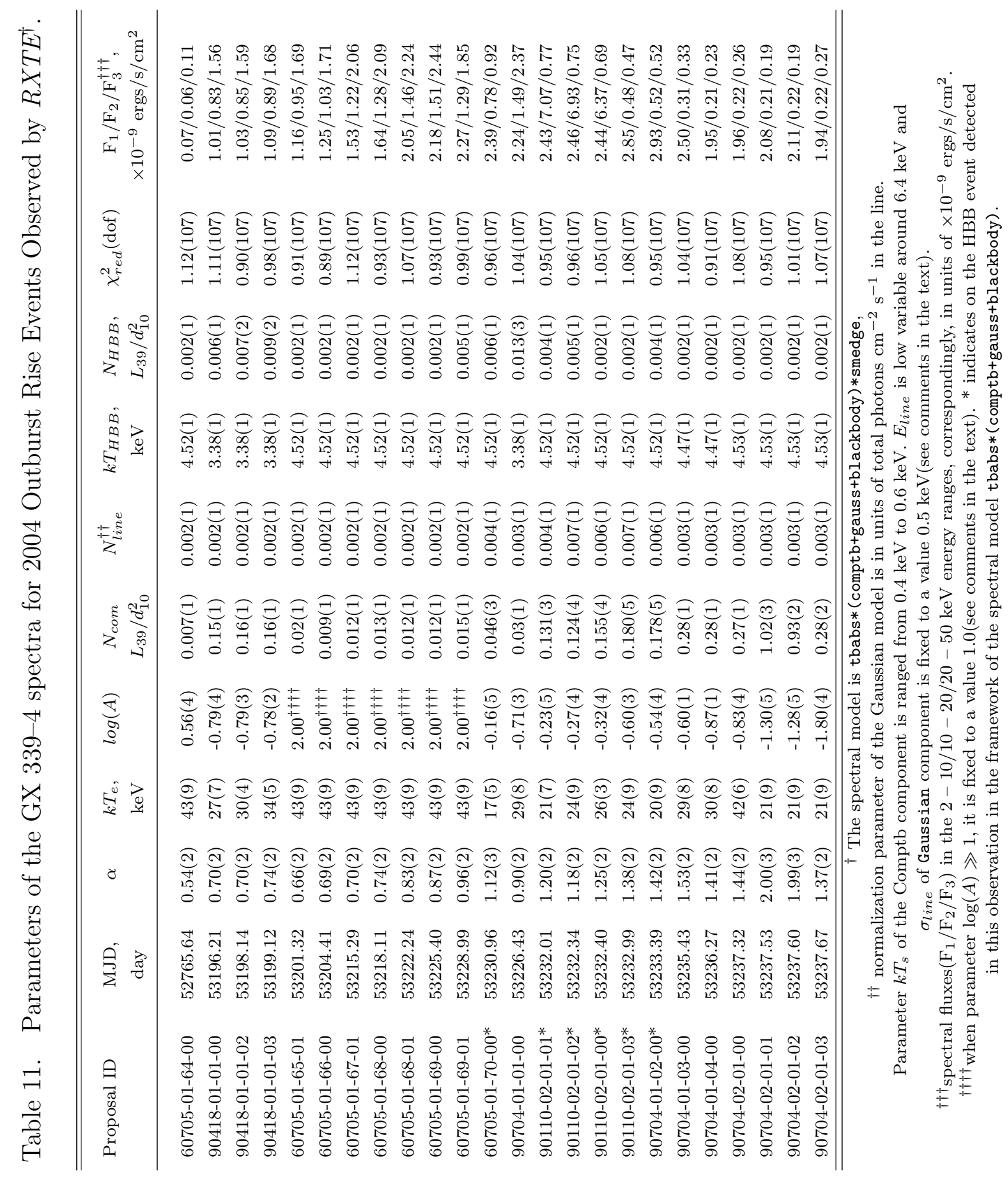




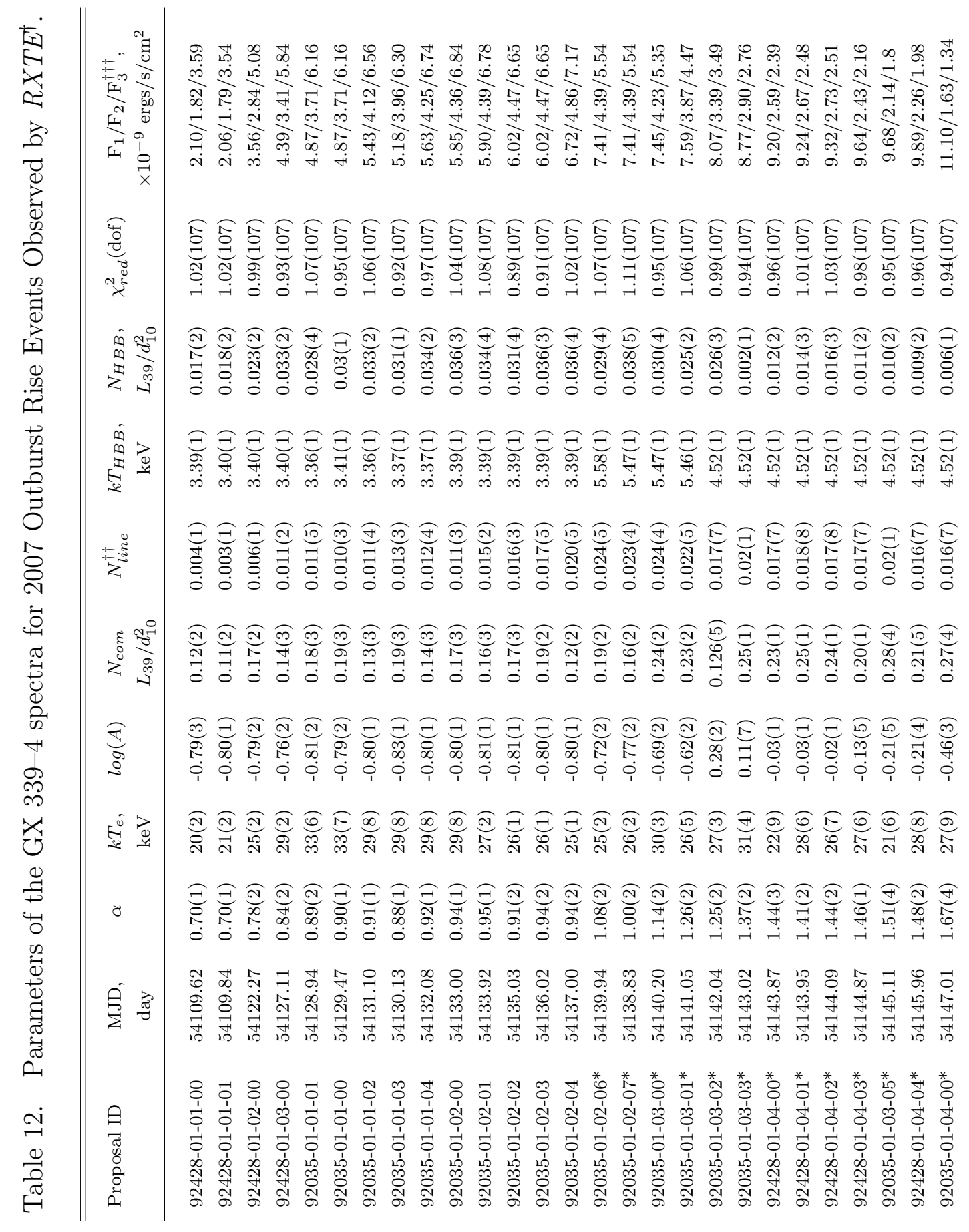




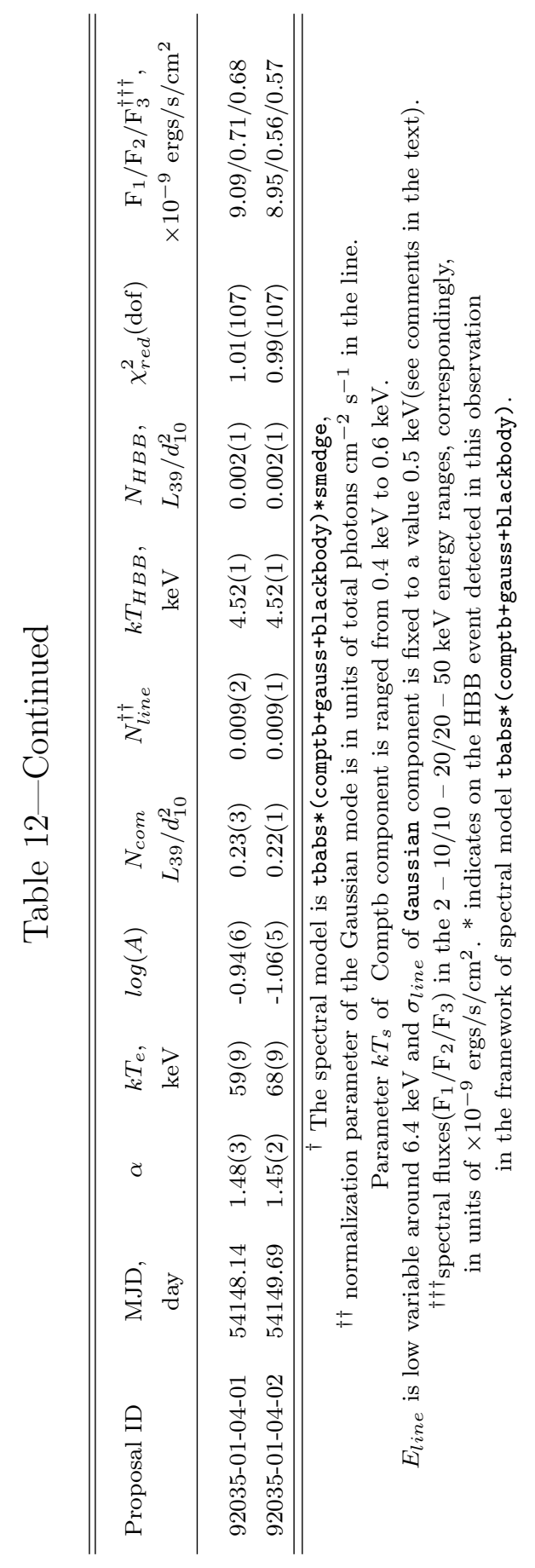


Table 13. Spectral best-fit parameters of the GRS 1915+105 observations in the 3-150 keV energy range by RXTE/PCA + HEXTE data using a model tbabs* (comptb+gauss+bbody)*smedge. Parameter errors (put in parenthesis) correspond to $1 \sigma$ confidence level.

\begin{tabular}{|c|c|c|c|c|c|c|c|c|c|c|c|}
\hline $\begin{array}{l}\text { Observational } \\
\text { ID }\end{array}$ & $\begin{array}{l}\text { MJD, } \\
\text { day }\end{array}$ & $\Gamma$ & $\begin{array}{l}k T_{s} \\
(\mathrm{keV})\end{array}$ & $\log (A)$ & $\begin{array}{l}N_{\text {com }} \\
L_{39} / d_{10}^{2}\end{array}$ & $\begin{array}{l}k T_{e} \\
(\mathrm{keV})\end{array}$ & $\begin{array}{l}\mathrm{kT}_{H B B} \\
(\mathrm{keV})\end{array}$ & $\begin{array}{l}\mathrm{N}_{H B B} \\
L_{39} / d_{10}^{2}\end{array}$ & $\begin{array}{l}\mathrm{E}_{\text {line }} \\
(\mathrm{keV})\end{array}$ & $\mathrm{N}_{\text {line }}$ & $\chi_{r e d}^{2}(d o f)$ \\
\hline $91701-01-31-00$ & 53646.76 & $1.86(9)$ & $1.36(7)$ & $2.00^{\dagger \dagger}$ & $0.2(1)$ & $4.7(5)$ & $4.5(1)$ & $0.002(1)$ & $6.70(2)$ & $0.02(1)$ & $1.20(78)$ \\
\hline $90024-02-37-00$ & 53646.90 & $2.02(9)$ & $1.52(5)$ & $2.00^{\dagger \dagger}$ & $0.3(1)$ & $10(1)$ & $4.5(1)$ & $0.002(1)$ & $6.74(3)$ & $0.03(1)$ & $0.93(78)$ \\
\hline 91701-01-33-01 & 53659.98 & $2.4(1)$ & $1.90(5)$ & $2.00^{\dagger \dagger}$ & $0.3(1)$ & $26(1)$ & $4.5(1)$ & $0.002(1)$ & $6.76(3)$ & $0.03(1)$ & $1.16(78)$ \\
\hline $90024-02-38-00$ & 53661.62 & $2.00(9)$ & $1.50(6)$ & $2.00^{\dagger \dagger}$ & $0.39(5)$ & $12.7(9)$ & $4.5(1)$ & $0.002(1)$ & $6.69(2)$ & $0.02(1)$ & $1.07(78)$ \\
\hline $91701-01-33-00$ & 53661.70 & $2.33(9)$ & $1.83(8)$ & $2.00^{\dagger \dagger}$ & $0.53(7)$ & $3.1(1)$ & $4.5(1)$ & $0.002(1)$ & $6.74(8)$ & $0.04(1)$ & $1.06(78)$ \\
\hline 91701-01-34-00 & 53661.69 & $1.79(7)$ & $1.29(5)$ & $2.00^{\dagger \dagger}$ & $0.30(6)$ & $10.9(8)$ & $4.5(1)$ & $0.002(1)$ & $6.74(4)$ & $0.04(1)$ & $1.26(78)$ \\
\hline 90105-06-03-01 & 53694.91 & $2.3(1)$ & $1.81(5)$ & $-0.41(7)$ & $0.86(7)$ & $130(10)$ & $4.5(1)$ & $0.002(1)$ & $6.4(1)$ & $0.002(1)$ & $1.16(78)$ \\
\hline 90105-06-03-00 & 53695.04 & $2.22(8)$ & $1.72(4)$ & $-0.36(2)$ & $0.72(9)$ & $140(3)$ & $4.5(1)$ & $0.002(1)$ & $6.4(1)$ & $0.002(1)$ & $1.13(78)$ \\
\hline 90105-06-03-02 & 53695.31 & $2.3(1)$ & $1.80(4)$ & $-0.2(1)$ & $0.6(1)$ & $100(8)$ & $4.5(1)$ & $0.002(1)$ & $6.4(1)$ & $0.002(1)$ & $1.07(78)$ \\
\hline $90024-02-40-00$ & 53696.70 & $2.4(1)$ & $1.93(9)$ & $-0.30(2)$ & $0.7(1)$ & $5.4(7)$ & $4.5(1)$ & $0.002(1)$ & $6.7(1)$ & $0.10(4)$ & $1.02(78)$ \\
\hline 91701-01-34-01 & 53669.75 & $1.81(7)$ & $1.31(5)$ & $2.00^{\dagger \dagger}$ & $0.31(6)$ & $10.8(9)$ & $4.5(1)$ & $0.002(1)$ & $6.72(5)$ & $0.05(1)$ & $1.19(78)$ \\
\hline $91701-01-35-00$ & 53674.59 & $2.20(9)$ & $1.70(3)$ & $0.14(6)$ & $0.29(6)$ & $90(5)$ & $4.5(1)$ & $0.002(1)$ & $6.74(8)$ & $0.04(1)$ & $0.77(78)$ \\
\hline 91701-01-38-00 & 53696.56 & $2.34(6)$ & $1.84(1)$ & $-0.52(1)$ & $0.7(1)$ & $120(9)$ & $4.5(1)$ & $0.002(1)$ & $6.4(1)$ & $0.002(1)$ & $1.04(80)$ \\
\hline 91701-01-38-01 & 53696.63 & $2.28(8)$ & $1.78(1)$ & $-0.34(5)$ & $0.78(1)$ & $105(8)$ & $4.5(1)$ & $0.002(1)$ & $6.4(1)$ & $0.002(1)$ & $1.28(80)$ \\
\hline 91701-01-40-00 & 53702.52 & $2.25(4)$ & $1.75(2)$ & $-0.03(1)$ & $0.43(1)$ & $100(15)$ & $4.5(1)$ & $0.002(1)$ & $6.4(1)$ & $0.002(1)$ & $1.07(80)$ \\
\hline 91701-01-39-00 & 53703.57 & $2.22(3)$ & $1.72(4)$ & $0.4(1)$ & $0.43(2)$ & $129(11)$ & $4.5(1)$ & $0.002(1)$ & $6.4(1)$ & $0.002(1)$ & $0.75(80)$ \\
\hline 91701-01-39-01 & 53703.63 & $2.03(4)$ & $1.53(3)$ & $0.34(9)$ & $0.37(1)$ & $30(4)$ & $4.5(1)$ & $0.002(1)$ & $6.4(1)$ & $0.002(1)$ & $0.96(80)$ \\
\hline 91701-01-41-00* & 53718.56 & $2.16(9)$ & $1.66(1)$ & $-1.91(8)$ & $0.3(2)$ & $2.5(2)$ & $4.0(1)$ & $2.61(5)$ & $8.02(9)$ & $0.09(1)$ & $0.93(75)$ \\
\hline 91701-01-42-00* & 53723.47 & $2.3(1)$ & $1.79(1)$ & $-1.83(5)$ & $0.34(9)$ & $1.9(5)$ & $3.82(9)$ & $1.50(2)$ & $7.89(7)$ & $0.2(1)$ & $0.76(75)$ \\
\hline 91701-01-42-01* & 53723.54 & $2.24(8)$ & $1.74(2)$ & $-1.79(8)$ & $0.3(1)$ & $2.2(3)$ & $4.01(9)$ & $1.62(1)$ & $7.9(1)$ & $0.08(1)$ & $0.95(75)$ \\
\hline $91701-01-43-00 *$ & 53730.41 & $2.28(7)$ & $1.78(1)$ & $-1.77(9)$ & $0.36(8)$ & $5.6(1)$ & $3.9(2)$ & $2.04(5)$ & $7.96(9)$ & $0.10(6)$ & $0.94(75)$ \\
\hline 91701-01-43-01* & 53730.51 & $2.21(9)$ & $1.71(2)$ & $-1.8(1)$ & $0.36(7)$ & $110(12)$ & $3.7(1)$ & $1.9(1)$ & $7.9(1)$ & $0.42(9)$ & $0.90(75)$ \\
\hline 90024-02-43-00 & 53753.51 & $2.17(5)$ & $1.67(5)$ & $2.00^{\dagger \dagger}$ & $0.69(8)$ & $35(4)$ & $4.5(1)$ & $0.002(1)$ & $6.67(3)$ & $0.03(2)$ & $0.75(78)$ \\
\hline 91701-01-49-00* & 53771.51 & $1.89(8)$ & $1.39(1)$ & $-1.8(1)$ & $0.24(9)$ & $40(8)$ & $4.51(9)$ & $2.31(4)$ & $7.81(9)$ & $0.08(2)$ & $0.95(75)$ \\
\hline $91701-01-46-00^{*}$ & 53778.30 & $2.14(8)$ & $1.64(3)$ & $0.01(1)$ & $0.40(2)$ & $6.6(5)$ & $4.0(3)$ & $2.36(5)$ & $5.96(4)$ & $0.09(3)$ & $0.74(75)$ \\
\hline 91701-01-50-00* & 53778.30 & $1.88(6)$ & $1.38(1)$ & $-1.7(1)$ & $0.25(4)$ & $26(5)$ & $4.00(7)$ & $1.63(5)$ & $7.83(8)$ & $0.2(1)$ & $0.81(75)$ \\
\hline 91701-01-22-00 & 53794.31 & $1.3(1)$ & $0.8(3)$ & $2.00^{\dagger \dagger}$ & $0.14(2)$ & $15(1)$ & $4.5(1)$ & $0.002(1)$ & $6.71(4)$ & $0.04(1)$ & $0.71(78)$ \\
\hline 92092-01-01-01 & 53704.94 & $2.3(1)$ & $1.78(8)$ & $2.00^{\dagger \dagger}$ & $0.34(7)$ & $4.2(6)$ & $4.5(1)$ & $0.002(1)$ & $6.71(8)$ & $0.08(2)$ & $0.94(78)$ \\
\hline 92092-01-01-00 & 53705.06 & $2.13(6)$ & $1.63(5)$ & $2.00^{\dagger \dagger}$ & $0.3(1)$ & $17(1)$ & $4.5(1)$ & $0.002(1)$ & $6.6(1)$ & $0.13(4)$ & $0.67(78)$ \\
\hline 92092-02-01-00 & 53705.84 & $2.2(1)$ & $1.67(5)$ & $2.00^{\dagger \dagger}$ & $0.3(2)$ & $3.6(2)$ & $4.5(1)$ & $0.002(1)$ & $6.72(3)$ & $0.03(1)$ & $0.92(78)$ \\
\hline 92092-02-01-01 & 53706.00 & $1.93(4)$ & $1.43(4)$ & $2.00^{\dagger \dagger}$ & $0.2(1)$ & $3.1(5)$ & $4.5(1)$ & $0.002(1)$ & $6.76(8)$ & $0.27(2)$ & $0.75(78)$ \\
\hline 92092-03-01-00 & 53707.68 & $2.24(9)$ & $1.74(3)$ & $-0.59(5)$ & $0.75(9)$ & $99(3)$ & $4.5(1)$ & $0.002(1)$ & $6.7(1)$ & $0.10(8)$ & $0.75(78)$ \\
\hline
\end{tabular}

$\dagger^{\dagger}$ when the parameter $\log (A) \gg 1$ then this parameter is fixed at a value of 2 (see comments in the text). 
Table 14. The HBB parameters for observational time segments for a simultaneous PCA + HEXTE/RXTE observation of GRS 1915+105 (91701-01-49-00).

\begin{tabular}{ccccc|ccccc}
\hline \hline Interval & $\begin{array}{c}\text { Start Time } \\
\text { Number }\end{array}$ & $\begin{array}{c}\text { Exp } \\
(\mathrm{s})\end{array}$ & $\begin{array}{c}\mathrm{EW} \text { of HBB, } \\
(\mathrm{eV})\end{array}$ & $\begin{array}{c}\mathrm{kT}_{H B B} \\
(\mathrm{keV})\end{array}$ & $\begin{array}{c}\text { Interval } \\
\text { Number }\end{array}$ & $\begin{array}{c}\text { Start Time } \\
(\mathrm{s})\end{array}$ & $\begin{array}{c}\text { Exp } \\
(\mathrm{s})\end{array}$ & $\begin{array}{c}\text { EW of HBB, } \\
(\mathrm{eV})\end{array}$ & $\begin{array}{c}\mathrm{kT} \mathrm{T}_{H B B} \\
(\mathrm{keV})\end{array}$ \\
\hline & 381759283.4 & 15 & $610 \pm 70$ & $4.47 \pm 0.09$ & 17 & 381760291.4 & 31 & $70 \pm 20$ & $4.5 \pm 0.2$ \\
1 & 381759331.4 & 31 & $590 \pm 60$ & $4.5 \pm 0.1$ & 18 & 381760355.4 & 31 & $50 \pm 10$ & $4.7 \pm 0.2$ \\
3 & 381759395.4 & 31 & $550 \pm 50$ & $4.5 \pm 0.2$ & 19 & 381760419.4 & 31 & $60 \pm 10$ & $4.5 \pm 0.1$ \\
4 & 381759459.4 & 31 & $530 \pm 50$ & $4.5 \pm 0.1$ & 20 & 381760483.4 & 31 & $80 \pm 30$ & $4.5 \pm 0.2$ \\
5 & 381759523.4 & 31 & $510 \pm 50$ & $4.6 \pm 0.1$ & 21 & 381760547.4 & 31 & $50 \pm 10$ & $4.6 \pm 0.1$ \\
6 & 381759587.4 & 31 & $500 \pm 60$ & $4.5 \pm 0.2$ & 22 & 381760611.4 & 31 & $40 \pm 10$ & $4.5 \pm 0.2$ \\
7 & 381759651.4 & 31 & $510 \pm 50$ & $4.5 \pm 0.2$ & 23 & 381760675.4 & 31 & $50 \pm 10$ & $4.7 \pm 0.2$ \\
8 & 381759715.4 & 31 & $670 \pm 80$ & $4.5 \pm 0.1$ & 24 & 381760739.4 & 31 & $50 \pm 6$ & $4.5 \pm 0.2$ \\
9 & 381759779.4 & 31 & $330 \pm 50$ & $4.5 \pm 0.2$ & 25 & 381760803.4 & 31 & $60 \pm 20$ & $4.5 \pm 0.1$ \\
10 & 381759843.4 & 31 & $330 \pm 50$ & $4.6 \pm 0.2$ & 26 & 381760867.4 & 31 & $50 \pm 8$ & $4.5 \pm 0.2$ \\
11 & 381759907.4 & 31 & $330 \pm 50$ & $4.6 \pm 0.2$ & 27 & 381760931.4 & 31 & $30 \pm 5$ & $4.7 \pm 0.2$ \\
12 & 381759971.4 & 31 & $50 \pm 20$ & $4.6 \pm 0.1$ & 28 & 381760995.4 & 31 & $40 \pm 10$ & $4.5 \pm 0.1$ \\
13 & 381760035.4 & 31 & $40 \pm 10$ & $4.5 \pm 0.2$ & 29 & 381761059.4 & 31 & $40 \pm 10$ & $4.5 \pm 0.2$ \\
14 & 381760099.4 & 31 & $45 \pm 8$ & $4.5 \pm 0.2$ & 30 & 381761123.4 & 31 & $50 \pm 20$ & $4.6 \pm 0.2$ \\
15 & 381760163.4 & 31 & $40 \pm 20$ & $4.5 \pm 0.1$ & 31 & 381761187.4 & 31 & $50 \pm 10$ & $4.5 \pm 0.2$ \\
16 & 381760227.4 & 31 & $30 \pm 5$ & $4.6 \pm 0.2$ & 32 & 381761251.4 & 31 & $50 \pm 10$ & $4.6 \pm 0.1$ \\
\hline
\end{tabular}

Table 15. Spectral best-fit parameters of the GRS $1915+105$ observations in the $3-$ $100 \mathrm{keV}$ energy range using a model tbabs* (comptb+gauss+bbody)*smedge for 91701-01-49-00 observation. Parameter errors (put in parenthesis) correspond to $1 \sigma$ confidence level.

\begin{tabular}{|c|c|c|c|c|c|c|c|c|c|c|}
\hline $\begin{array}{l}\text { Interval } \\
\text { Number }\end{array}$ & $\begin{array}{c}\text { Start time }^{\dagger}(\mathrm{s}) \\
381700000+\end{array}$ & $\begin{array}{l}\alpha= \\
\Gamma-1\end{array}$ & $\log (A)$ & $\begin{array}{c}N_{\text {comp }} \\
\mathrm{keV}\end{array}$ & $\mathrm{kT}_{H B B}$ & $\begin{array}{c}\mathrm{N}_{H B B} \\
\mathrm{keV}\end{array}$ & $\mathrm{E}_{\text {line }}$ & $\begin{array}{c}\mathrm{N}_{\text {line }} \\
L_{39} / d_{10}^{2}\end{array}$ & Flux ${ }^{\dagger \dagger \dagger}$ & $\chi_{\text {red }}^{2}(d o f)$ \\
\hline 1 & 59283.4 & $1.70(3)$ & $0.35(9)$ & $0.22(1)$ & $4.47(9)$ & $3.5(1)$ & $6.4(1)$ & $0.019(3)$ & 9.35 & $1.05(112)$ \\
\hline 2 & 59331.4 & $1.69(2)$ & $-0.1(2)$ & $0.21(4)$ & $4.5(1)$ & $3.31(9)$ & $6.62(7)$ & $0.017(4)$ & 3.47 & $1.01(112)$ \\
\hline 3 & 59395.4 & $1.67(3)$ & $0.02(1)$ & $0.19(2)$ & $4.5(2)$ & $3.4(2)$ & $6.59(6)$ & $0.016(5)$ & 3.59 & $1.09(112)$ \\
\hline 4 & 59459.4 & $1.67(3)$ & $0.77(4)$ & $0.20(6)$ & $4.5(1)$ & $3.2(1)$ & $6.62(7)$ & $0.018(4)$ & 3.37 & $1.08(112)$ \\
\hline 5 & 59529.4 & $1.68(4)$ & $0.16(5)$ & $0.19(8)$ & $4.6(1)$ & $3.6(1)$ & $6.69(7)$ & $0.02(1)$ & 3.57 & $1.02(113)$ \\
\hline 6 & 59587.4 & $1.68(3)$ & $2.0^{\dagger \dagger}$ & $0.18(7)$ & $4.5(2)$ & $3.5(1)$ & $6.78(6)$ & $0.015(5)$ & 3.42 & $1.01(114)$ \\
\hline 7 & 59651.4 & $1.68(5)$ & $1.9(0.6)$ & $0.18(8)$ & $4.5(2)$ & $3.4(2)$ & $6.71(7)$ & $0.019(4)$ & 3.45 & $1.07(113)$ \\
\hline 8 & 59715.4 & $1.67(4)$ & $0.65(1)$ & $0.18(2)$ & $4.5(2)$ & $3.6(1)$ & $6.56(6)$ & $0.016(5)$ & 3.81 & $1.08(113)$ \\
\hline 9 & 59779.4 & $1.67(5)$ & $0.15(9)$ & $0.19(6)$ & $4.5(2)$ & $3.5(1)$ & $6.48(6)$ & $0.018(3)$ & 3.43 & $1.02(113)$ \\
\hline 10 & 59843.4 & $1.68(3)$ & $0.06(1)$ & $0.18(2)$ & $4.6(2)$ & $3.4(1)$ & $6.47(5)$ & $0.017(2)$ & 3.48 & $1.04(113)$ \\
\hline 11 & 59907.4 & $1.67(4)$ & $-0.46(1)$ & $0.18(1)$ & $4.6(2)$ & $3.5(2)$ & $6.5(1)$ & $0.020(5)$ & 3.41 & $0.99(113)$ \\
\hline
\end{tabular}

\footnotetext{
${ }^{\dagger}$ time is presented as a specific $R X T E$ time; ${ }^{\dagger}$ if the parameter $\log (A) \gg 1$ then this parameter is fixed at a value of 2 (see
} comments in the text); ${ }^{\dagger \dagger}$ spectral flux in the $3-100$ energy range in units of $10^{-9} \mathrm{ergs} / \mathrm{s} / \mathrm{cm}^{2}$. 
Table 16. The best-fit parameters of the simultaneous PCA/RXTE (90401-01-01-01) and ISGRI/IBIS/INTEGRAL observation of SS 433 in the $3-150 \mathrm{keV}$ energy range (rev. 336) on March $24-27,2004^{\dagger}$. Errors correspond to $1 \sigma$ confidence level.

\begin{tabular}{|c|c|c|c|c|c|c|c|c|c|c|c|c|}
\hline $\begin{array}{l}\text { MJD, } \\
\text { day }\end{array}$ & $\begin{array}{c}\alpha= \\
\Gamma-1\end{array}$ & $\log (A)$ & $\begin{array}{c}\mathrm{N}_{\text {com }} \\
L_{39} / d_{10}^{2}\end{array}$ & $\begin{array}{c}\mathrm{E}_{\text {line } 1} \\
\mathrm{keV}\end{array}$ & $\begin{array}{c}\sigma_{\text {line } 1} \\
\mathrm{keV}\end{array}$ & $N_{\text {line } 1}$ & $\begin{array}{c}\mathrm{E}_{\text {line2 }}, \\
\mathrm{keV}\end{array}$ & $\mathrm{N}_{\text {line2 }}^{\dagger \dagger}$ & $\begin{array}{c}\mathrm{kT}_{H B B} \\
\mathrm{keV}\end{array}$ & $\begin{array}{l}\mathrm{N}_{H B B} \\
L_{36} / d_{10}^{2}\end{array}$ & $\begin{array}{c}\mathrm{EW}_{H B B} \\
\mathrm{keV}\end{array}$ & $\chi_{\text {red }}^{2}$ (d.o.f.) \\
\hline 53076.78 & $1.3(2)$ & $0.5(2)$ & $3.3(3)$ & $7.23(4)$ & $0.96(7)$ & $4.74(6)$ & $9.2(4)$ & $0.14(4)$ & $5.9(2)$ & $3.6(3)$ & $0.4(4)$ & $1.17(77)$ \\
\hline
\end{tabular}

${ }^{\dagger}$ The spectral model is tbabs* (comptb+Gaussian1+Gaussian2+bbody)*smedge; ${ }^{\dagger \dagger}$ normalization parameter of Gaussian1 and Gaussian2 components is in units of $10^{-3} \times$ total photons $\mathrm{cm}^{-2} \mathrm{~s}^{-1}$ in the line, $\sigma_{\text {line } 2}$ of Gaussian2 component is fixed at a value $0.01 \mathrm{keV}$ (see comments in the text).

Table 17. The best-fit parameters for the BeppoSAX observations of V4641 Sgr in the 0.3 - $150 \mathrm{keV}$ energy range ${ }^{\dagger}$. Errors correspond to $1 \sigma$ confidence level.

\begin{tabular}{|c|c|c|c|c|c|c|c|c|c|c|}
\hline $\begin{array}{c}\text { Observational } \\
\text { ID }\end{array}$ & $\begin{array}{l}\text { MJD, } \\
\text { day }\end{array}$ & $\begin{array}{c}\alpha= \\
\Gamma-1\end{array}$ & $\log (A)$ & $\mathrm{N}_{\mathrm{com}}^{\dagger \dagger}$ & $\begin{array}{c}\mathrm{E}_{\text {laor }} \\
\mathrm{keV}\end{array}$ & $\mathrm{N}_{\text {laor }}^{\dagger \dagger}$ & $\begin{array}{c}\mathrm{kT}_{H B B} \\
\mathrm{keV}\end{array}$ & $\mathrm{N}_{H B B}^{\dagger \dagger}$ & $\mathrm{F}_{1} / \mathrm{F}_{2}$ & $\chi_{\text {red }}^{2}$ (d.o.f.) \\
\hline 205490061 & 51250.76 & $1.47(9)$ & $0.65(2)$ & $5.3(3)$ & $6.99(7)$ & $3.76(8)$ & $5.10(4)$ & $4.18(3)$ & $2.6 / 5.2$ & $1.09(157)$ \\
\hline
\end{tabular}

Table 18. The best-fit parameters for the RXTE observations of V4641 Sgr in the 3 $100 \mathrm{keV}$ energy range ${ }^{\dagger}$. Errors correspond to $1 \sigma$ confidence level.

\begin{tabular}{|c|c|c|c|c|c|c|c|c|c|}
\hline $\begin{array}{l}\text { Observational } \\
\text { ID }\end{array}$ & $\begin{array}{l}\text { MJD } \\
\text { day }\end{array}$ & $\begin{array}{c}\alpha= \\
\Gamma-1\end{array}$ & $\log (A)$ & $\begin{array}{c}\mathrm{N}_{c o m}^{\dagger \dagger} \\
L_{39} / d_{10}^{2}\end{array}$ & $\begin{array}{c}\mathrm{E}_{\text {line }} \\
\mathrm{keV}\end{array}$ & $\mathrm{N}_{\text {line }}^{\dagger \dagger}$ & $\begin{array}{c}\mathrm{kT}_{H B B} \\
\mathrm{keV}\end{array}$ & $\mathrm{N}_{H B B}^{\dagger \dagger}$ & $\chi_{\text {red }}^{2}$ (d.o.f.) \\
\hline 404030-01-01-00 & 51436.8 & $1.93 \pm 0.08$ & $-3.1 \pm 0.1$ & $129 \pm 15$ & $6.7 \pm 0.1$ & $0.28 \pm 0.03$ & $4.5(1)$ & $0.002(1)$ & $0.93(78)$ \\
\hline 404030-01-02-00 & 51437.1 & $1.87 \pm 0.02$ & $-3.1 \pm 0.2$ & $150 \pm 12$ & $6.5 \pm 0.2$ & $0.59 \pm 0.02$ & $4.6(2)$ & $5.2(1)$ & $0.98(76)$ \\
\hline 404030-01-03-00 & 51237.06 & $1.91 \pm 0.09$ & $-2.7 \pm 0.1$ & $89 \pm 19$ & $6.6 \pm 0.1$ & $0.58 \pm 0.09$ & $4.5(1)$ & $0.002(1)$ & $0.92(78)$ \\
\hline 404030-01-03-01G & 51237.13 & $1.8 \pm 0.06$ & $-1.68 \pm 0.09$ & $54 \pm 10$ & $6.5 \pm 0.1$ & $0.41 \pm 0.05$ & $4.5(1)$ & $5.8(2)$ & $0.82(76)$ \\
\hline 404030-01-04-00 & 51237.7 & $1.73 \pm 0.05$ & $-2.87 \pm 0.06$ & $70 \pm 15$ & $6.4 \pm 0.1$ & $0.63 \pm 0.07$ & $4.8(3)$ & $6.1(1)$ & $0.89(76)$ \\
\hline 404030-01-04-01 & 51237.9 & $1.40 \pm 0.02$ & $-3.42 \pm 0.19$ & $5.5 \pm 1.0$ & $6.4 \pm 0.2$ & $0.11 \pm 0.09$ & $4.5(1)$ & $0.002(1)$ & $1.03(78)$ \\
\hline 404030-01-07-00G & 51238.9 & $0.89 \pm 0.02$ & $-0.08 \pm 0.01$ & $0.29 \pm 0.07$ & $6.4 \pm 0.2$ & $0.11 \pm 0.09$ & $4.9(1)$ & $5.9(3)$ & $1.07(76)$ \\
\hline
\end{tabular}

$\dagger$ The spectral model is tbabs* (comptb+gauss+blackbody)*smedge,

$\dagger \dagger$ normalization parameter of blackbody and the Comptb components are in units of $10^{-3} \times * L_{39} / d_{10}^{2}$, normalization parameter of the gaussian model is in units of $10^{-3} \times$ total photons $\mathrm{cm}^{-2} \mathrm{~s}^{-1}$ in line. 